\title{
The hearing hippocampus
}

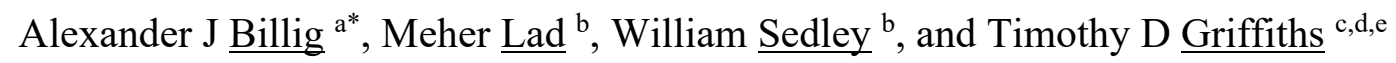

a. UCL Ear Institute, University College London, London, UK

b. Translational and Clinical Research Institute, Newcastle University Medical School, Newcastle upon Tyne, UK

c. Biosciences Institute, Newcastle University Medical School, Newcastle upon Tyne, UK

d. Wellcome Centre for Human Neuroimaging, UCL Queen Square Institute of Neurology, University College London, London, UK

e. Human Brain Research Laboratory, Department of Neurosurgery, University of Iowa Hospitals and Clinics, Iowa City, USA

* For correspondence: a.billig@ucl.ac.uk

\section{Preprint notes}

- This version 9th March 2022 (added funding source, removed two unpublished references)

- $\quad$ Not yet peer-reviewed 


\begin{abstract}
The hippocampus has a well-established role in spatial and episodic memory but a broader function has been proposed including aspects of perception and relational processing. Neural bases of sound analysis have been described in the pathway to auditory cortex, but wider networks supporting auditory cognition are still being established. We review what is known about the role of the hippocampus in processing auditory information, and how the hippocampus itself is shaped by sound. In examining imaging, recording, and lesion studies in species from rodents to humans, we uncover a hierarchy of hippocampal responses to sound including during passive exposure, active listening, and the learning of associations between sounds and other stimuli. We describe how the hippocampus' connectivity and computational architecture allow it to track and manipulate auditory information - whether in the form of speech, music, or environmental, emotional, or phantom sounds. Functional and structural correlates of auditory experience are also identified. The extent of auditory-hippocampal interactions is consistent with the view that the hippocampus makes broad contributions to perception and cognition, beyond spatial and episodic memory. More deeply understanding these interactions may unlock applications including entraining hippocampal rhythms to support cognition, and intervening in links between hearing loss and dementia.
\end{abstract}




\section{Introduction and motivation}

Given the two most well-known functions of the hippocampus - supporting episodic memory in humans (Scoville and Milner, 1957; Squire and Zola-Morgan, 1991) and spatial navigation in animals (O’Keefe and Dostrovsky, 1971) - a review of this structure in relation to sound may seem an unlikely exercise. In humans, cortical circuits underlying auditory perception and cognition are largely found in lateral, rather than medial temporal lobe structures (Bizley and Cohen, 2013; Griffiths and Warren, 2004; Rauschecker and Tian, 2000; Schnupp et al., 2013). Although the hippocampus has access to highly processed information from all sensory modalities, it is often conceptualized as sitting atop a visual cortical hierarchy (Felleman and Van Essen, 1991; Turk-Browne, 2019) and its role in auditory memory in primates has been challenged on anatomical and functional grounds (Fritz et al., 2005; Munoz-Lopez et al., 2010).

However, theories of hippocampal function have long extended beyond episodic memory and spatial navigation (Cohen and Eichenbaum, 1993; Kimble, 1968; Papez, 1937). One idea is that the hippocampus is important for the binding of arbitrary relations and mediating their flexible expression (Lisman et al., 2017; but see O'Keefe and Krupic, 2021). This extended job description encompasses linking multimodal objects with a spatiotemporal, environmental, or cognitive context to form episodic memories (Yonelinas et al., 2019), supporting short-term memory (Hannula and Ranganath, 2008; Pertzov et al., 2013), associating disparate elements of a scene (Graham et al., 2010; Maguire and Mullally, 2013; Olsen et al., 2012), structuring conceptual knowledge (Behrens et al., 2018), and forming predictions (Stachenfeld et al., 2017). Strong versions of such accounts might allow for involvement of the hippocampus in a wide range of situations involving auditory information, such as binding acoustic features into a perceptual whole, anticipating the continuation of sound sequences, and "mental navigation" along sequences of auditory stimuli. We shall see that the computational circuitry of the hippocampus is well suited for operating on information that is organized in time - such as that carried by acoustic signals. In light of this extended proposed scope for hippocampal function, a full account of the auditory system should at least consider this structure.

There are also practical and clinical motivations for understanding interactions between sound and the hippocampus. Auditory stimulation can entrain hippocampal rhythms, with implications for enhancing memory and mitigating cognitive decline (Derner et al., 2018; Harrington and Cairney, 2021; Martorell et al., 2019). Auditory signals interact with hippocampal memories, an effect that could be harnessed to boost learning (Cousins et al., 2016; Crowley et al., 2019) or target pathological memories in a clinical setting (Ressler et al., 2021). Hippocampal structure and function are also shaped by experience, raising the question of how auditory expertise and deprivation affect the hippocampus, for example playing a role in tinnitus (Kraus and Canlon, 2012; L. Zhang et al., 2018) or mediating a link between hearing loss and dementia (Griffiths et al., 2020; Livingston et al., 2017). Addressing these issues and tapping into therapeutic potential requires a consolidation of what we know about pathways that carry information between auditory sites and hippocampus.

Recognizing the relative preservation of hippocampal anatomy and physiology across species, and the advances in understanding function this affords (Buffalo, 2015; Clark and Squire, 2013; Cohen and Eichenbaum, 1993; Witter and Amaral, 2021), we include studies from rodents to primates. Electrophysiological and neuroimaging data are considered alongside neuropsychological and animal lesion work. We begin by outlining the anatomy of 
the hippocampus and the pathways connecting it with canonical auditory structures. We then characterize hippocampal responses to meaningless sounds, going on to consider how these change as sounds acquire taskrelevance or signal value. Circumstances under which the hippocampus supports different types of association are set out, with a focus on interactions between processing of sound, time, and space. This leads to a consideration of how the hippocampus might support the formation and retrieval of objects, scenes, and memories that are purely auditory. We cover the special cases of speech and music, emotional sounds and phantom percepts, then set out how auditory experience - and its absence - affects hippocampal structure and function.

Dominant accounts of hippocampal function, as well as key physiological properties, are briefly introduced as required, but readers are referred to detailed reviews (and for a short primer might consult Knierim (2015)). Known computational principles of the hippocampus are described to the extent that they can account for auditory data. A key question throughout is the extent to which hippocampal involvement in sound processing is secondary to or dependent on its established roles in episodic memory and spatial navigation. Another is the extent to which the hippocampus acts as an automatic processor of auditory information as opposed to any requirement for the information to be relevant to behavior. We shall find the concept of the hippocampus as a predictive map particularly useful for drawing together some of the findings. However, rather than attempting an integrated theory of the hippocampus through the prism of sound, our aim is to highlight the range of circumstances under which this structure processes and is shaped by auditory signals. In essence, we are not "claiming" the hippocampus as an auditory structure so much as examining how its computational architecture might be engaged in and altered by auditory tasks.

\section{Anatomy and auditory-hippocampal pathways}

Figure 1A shows the two interlocking gyri of the hippocampus - the cornu ammonis (including subfields CA1 and CA3) and dentate gyrus (DG) - extending postero-anteriorally in the medial temporal lobe of primates, and dorsoventrally just below neocortex in rodents. The major cortical input to this bilateral structure is from adjacent entorhinal cortex (ERC), which in primates forms part of the parahippocampal gyrus. A well-described pathway, the trisynaptic loop, projects from ERC through DG, CA3, and CA1 back to ERC, from where output is routed back to neocortex. There are also direct projections from ERC to CA1 (the monosynaptic pathway) and extensive recurrent connections within CA3. The hippocampus is also reciprocally connected via the fornix to thalamus, mammillary bodies, and the basal forebrain, as well as to amygdala, basal ganglia, cingulate, and frontal and parietal lobes. We will see later how computations associated with these pathways may be relevant to auditory processing.

Along with ERC, the parahippocampal gyrus in primates consists of perirhinal and parahippocampal (postrhinal in rodents) cortices, which connect to ERC from anterior and posterior directions respectively as shown in Figure 1B (for detailed connections see Burwell and Amaral, 1998; Garcia and Buffalo, 2020; Munoz-Lopez et al., 2010; Nilssen et al., 2019; van Strien et al., 2009 and other anatomical studies in Supplementary Table A). Fellemann \& Van Essen (1991) show hippocampus at the apex of a visual cortical hierarchy, with parahippocampal and perirhinal cortices exchanging information with high-order areas in the ventral visual pathway. The functional anatomy of the primate auditory system is less well mapped than that of vision, at least downstream of primary cortex beyond the lemniscal path from cochlea through the cochlear nucleus, inferior colliculus, and medial 


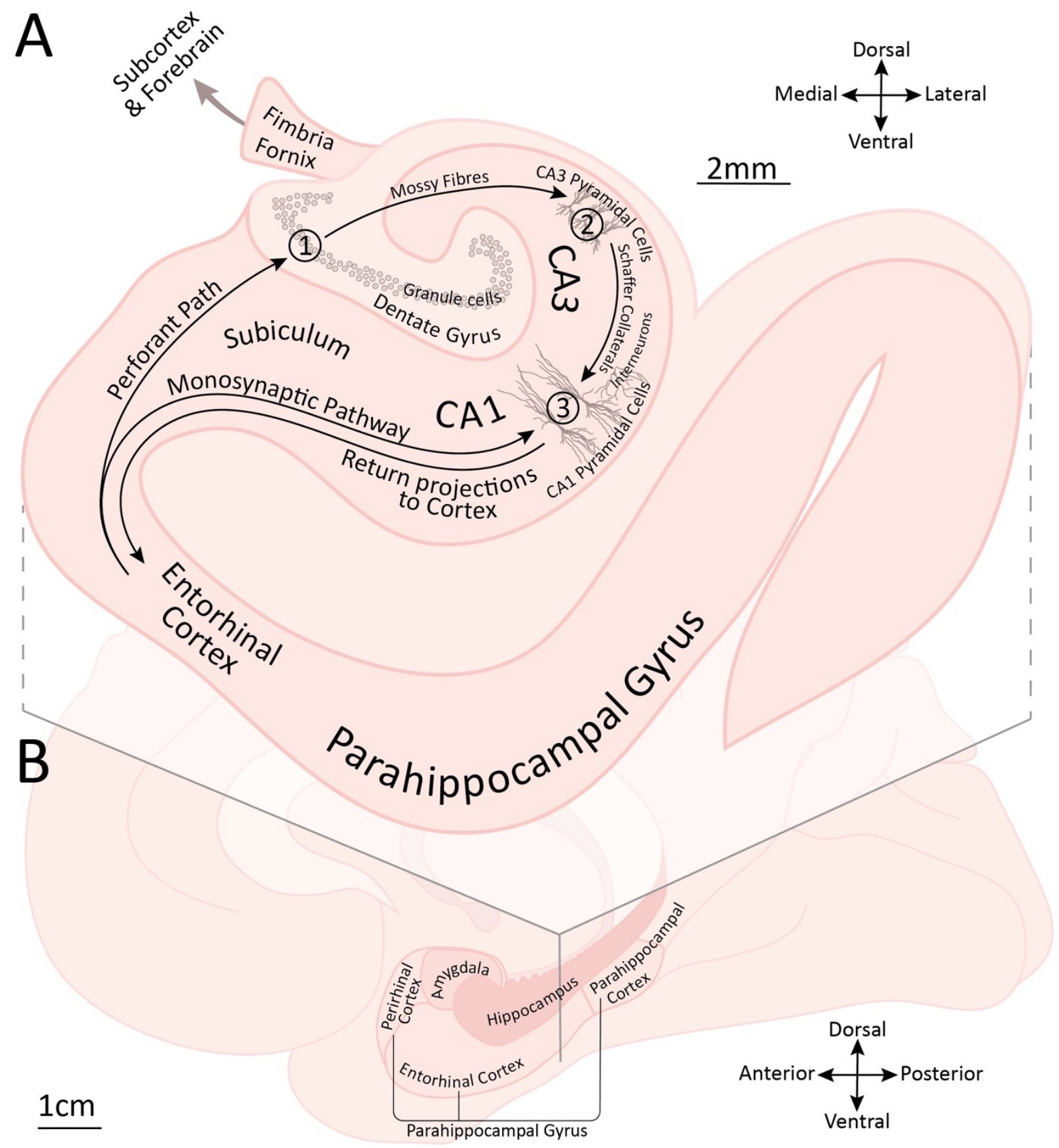

Fig. 1. (A) Coronal cross-section of human medial temporal lobe showing parahippocampal gyrus, entorhinal cortex, hippocampus with primary subfields, and key pathways. Pyramidal cells, interneurons and granule cells not shown to scale. (B) Medial sagittal view of human brain showing medial temporal lobe structures and indicating the position of the cross-section shown in A.

geniculate body of the thalamus. A dorsal pathway (sometimes termed a "where" stream due to its role in audiospatial processing) runs from posterior auditory cortex via parietal sites to dorsolateral prefrontal cortex (Rauschecker and Scott, 2016). More ventrally, a "what" stream courses anteriorly along superior temporal gyrus, superior temporal sulcus, and middle temporal gyrus, with features extracted and represented that are increasingly abstract and removed from the acoustic signal. This ventral pathway is often described as terminating in ventrolateral frontal cortex, however, at least in monkeys, additional projections from those anterior temporal sites via the temporal pole reach perirhinal, parahippocampal and entorhinal cortices, which in turn connect to 
hippocampus (Munoz-Lopez et al., 2015, 2010). Auditory information has multiple opportunities along this series of synapses to be integrated with that from other modalities. There are also somewhat more direct projections from association (belt or parabelt) cortex to entorhinal/perirhinal/parahippocampal cortex in macaques, and from primary cortex to perirhinal and entorhinal cortices in rodents, although these may be sparser for audition than in other sensory modalities (Amaral et al., 1983; Burwell and Amaral, 1998; Munoz-Lopez et al., 2010; Suzuki and Amaral, 1994; Yi et al., 2022). Efferent pathways from the medial temporal lobe trace similar routes back as the afferent connections described (Muñoz and Insausti, 2005; Tranel et al., 1988; Vaudano et al., 1991) but - in rodents at least - are supplemented by others, such as from hippocampus direct to primary auditory cortex (Cenquizca and Swanson, 2007) and even inferior colliculus (ref to follow).

In rodents, subcortical pathways also carry auditory information to the hippocampal formation, including one from cochlear nucleus via pontine nuclei and medial septum (Xiao et al., 2018; G.-W. Zhang et al., 2018), and another from thalamus via basolateral amygdala and entorhinal cortex (Bordi and LeDoux, 1994; LeDoux et al., 1985; Wahlstrom et al., 2018). That these bear auditory information is evidenced by early latency auditory responses at hippocampus and entorhinal cortex, prior to those occurring in auditory cortex, as described in Section 3. Anatomical connections from medial septum to entorhinal cortex have also been traced in non-human primates (Insausti et al., 1987). These subcortical pathways may provide fast, indiscriminate communication of the presence of sound, in contrast to slower cortical routes conveying more elaborated representations of a sound and its meaning, including after integrating information from other sensory modalities (Rolls, 1996). See Figure 2 for two ascending auditory-hippocampus pathways in the mouse, and Kraus \& Canlon (2012) for more detail on the interaction between the auditory system and other medial temporal lobe structures.

Establishing the extent to which both these standard and non-canonical pathways in rodents and non-human primates are mirrored in humans is difficult. In the absence of axonal tract tracing or post-mortem studies in humans, indirect measures of structural connectivity such as diffusion tensor imaging (DTI) can be informative. A neurosurgical atlas based on the large human connectome project dataset highlighted the absence of direct connections between medial and lateral temporal structures, although indirect pathways were functionally established that correspond to some of those outlined in non-human primates, such as from auditory association areas on the superior temporal gyrus to posterior parahippocampal fields then entorhinal cortex (Baker et al., 2018). One ultra high-resolution DTI study provided support for white matter tracts between hippocampus and both the temporal pole and planum polare, but not low-level auditory cortex (Maller et al., 2019). Another identified connections between hippocampus and a region of interest that included both auditory core and belt areas (Jang and Choi, 2022) in a majority of subjects. Differences across the results of these studies may relate to the thresholds used in the probabilistic tractography procedure.

DTI is a way of defining anatomical tracts that link different areas. In contrast, functional-connectivity analysis defines correlated time series between areas, based on which direct or indirect connections can be inferred. Such analysis of resting-state BOLD activity measured with fMRI has revealed distinct connectivity between different parts of the hippocampus and neocortical regions. Whereas activity in posterior hippocampus and parahippocampal cortex correlates with activity in lateral parietal cortex and midline sites, activity in anterior hippocampus and perirhinal/entorhinal cortex correlates with that in lateral temporal regions including superior temporal gyrus extending to temporal pole (Kahn et al., 2008; S.-F. Wang et al., 2016). Clustering of more 
temporally-resolved functional connectivity patterns derived from intracranially recorded high-frequency resting state activity found anterior and medial temporal sites, including hippocampus, to have the strongest coupling with auditory cortex (Banks et al., 2022). While that finding included primary auditory cortex, other resting state intracranial and fMRI studies of connections between medial temporal and sensory cortex have emphasized those

\section{A Canonical pathway}

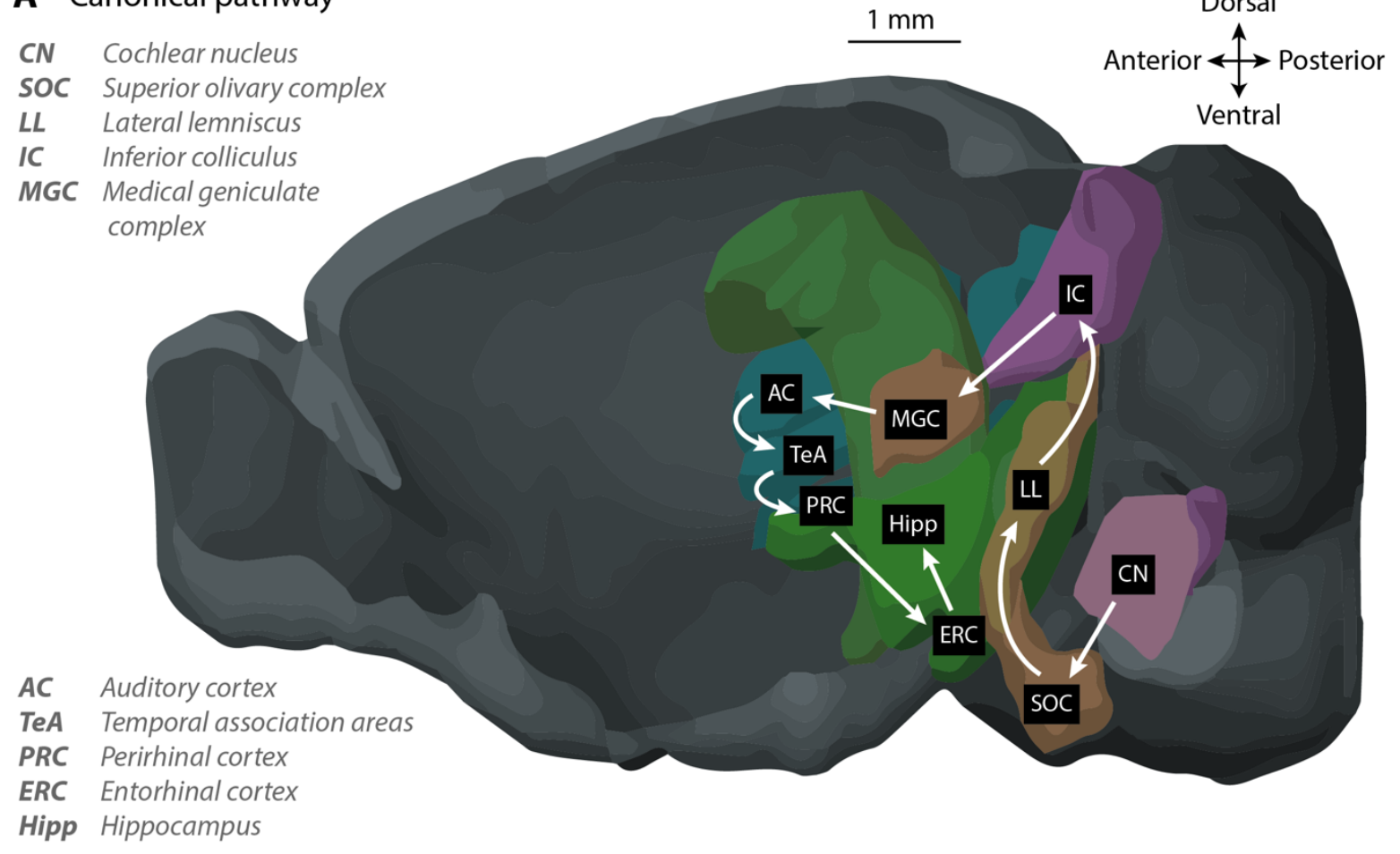

\section{B Rapid pathway}

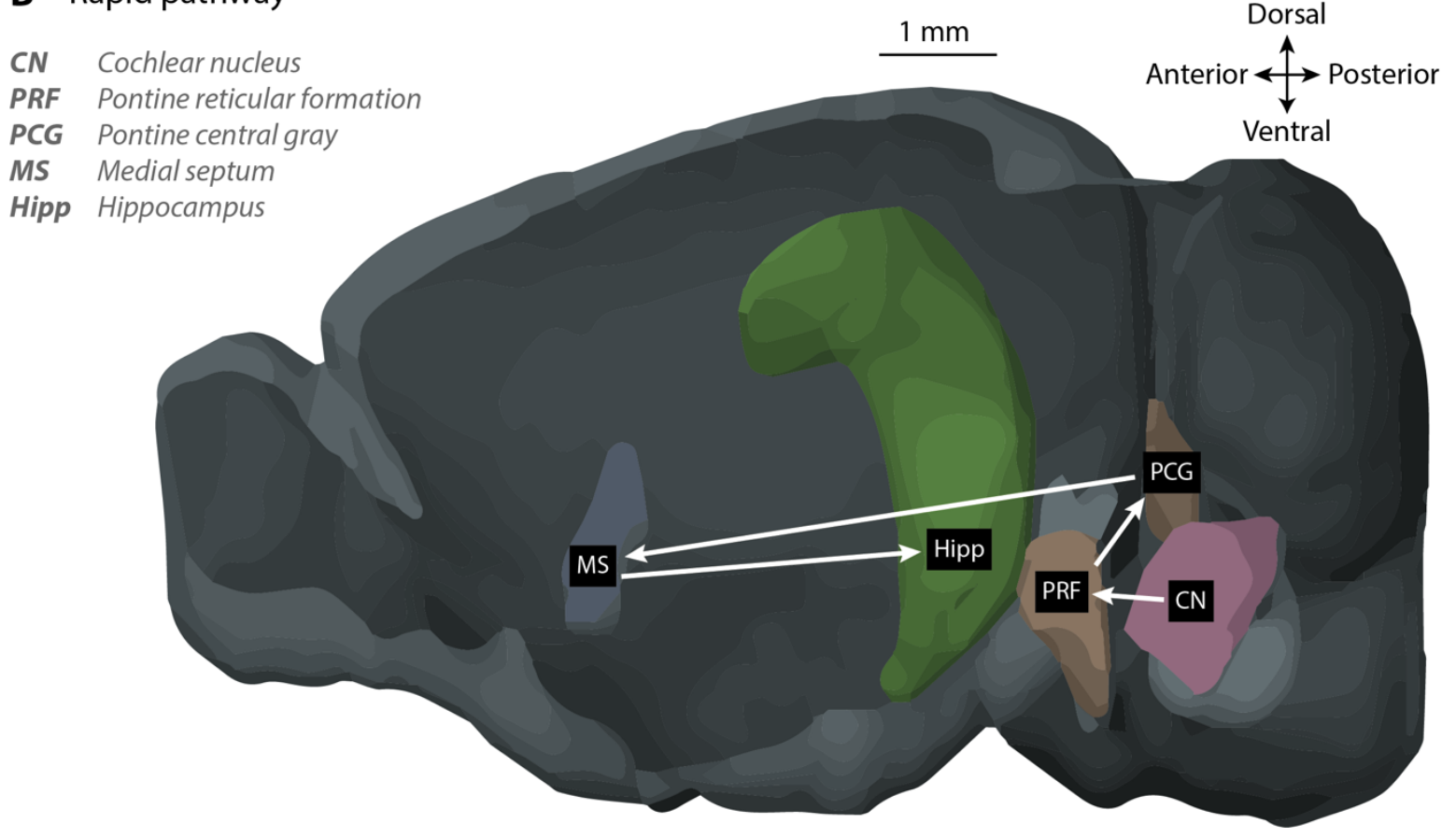

Fig. 2. Lateral view of mouse brain showing pathways from auditory brainstem structures to hippocampus. (A) Canonical pathway consisting of at least ten synapses and (B) A rapid five-synapse pathway. 
between hippocampus and association areas in humans, with the possible exception of olfaction, compared to the primary sites that dominate in rodents (Bergmann et al., 2016; Zhou et al., 2021).

Before describing auditory responses in hippocampus, we note that electrophysiological and fMRI studies coupled with electrical or optogenetic stimulation can also reveal pathways relevant to auditory processing. The hippocampus orchestrates activity across cortex, propagating theta oscillations (4-7 Hz in rodents) for temporal control of information processing, and sharp-wave ripples for memory consolidation (Buzsáki, 2015, 2002; see Section 8). For example, neurons in guinea pig inferior colliculus and auditory cortex phase lock to hippocampal theta both during spontaneous firing and in response to sound (Liberman et al., 2009; Pedemonte et al., 2001, 1996). In cats, brief electrical stimulation of dorsal hippocampus at a theta rate enhances auditory cortical responses to clicks presented immediately after the stimulation (Parmeggiani and Rapisarda, 1969). In addition, optogenetic stimulation of excitatory neurons in rat ventral hippocampus at a theta rate can elicit BOLD activity in sites including primary auditory cortex and boost responses there (as well as in inferior colliculus and medial geniculate body) to natural vocalizations but not artificial sounds (ref to follow).

Optogenetic stimulation at lower rates $(1 \mathrm{~Hz})$ in rat dentate gyrus increased resting state BOLD interhemispheric connectivity between primary auditory cortices (Chan et al., 2017). More rapid (40 Hz) optogenetic stimulation of excitatory cells in rat intermediate (but not dorsal) hippocampus produced elevated BOLD in auditory cortex (Weitz et al., 2015). Electrical stimulation at even faster rates in rabbits led to an increase in the amplitude of responses to clicks in motor cortex (Cazard and Buser, 1963), while other studies in cats found electrical stimulation of hippocampus leading to reduced auditory cortical responses to brief medial geniculate body electrical pulses (Redding, 1967), and reduced click responses in cerebellum (Fox et al., 1967) and hypothalamus (Feldman and Dafny, 1968). Single-pulse electrical stimulation of hippocampus even in the absence of an auditory stimulus elicits rapid responses in the auditory cortex not only of cats (Parmeggiani and Rapisarda, 1969) but also of humans. In the latter case these occur not only in auditory association areas on the lateral temporal lobe (Catenoix et al., 2011; Enatsu et al., 2015) but also primary auditory cortex in Heschl's gyrus, with initial responses as early as $10 \mathrm{~ms}$ (Rocchi et al., 2021). These electrical and optogenetic stimulation studies provide further evidence for anatomical and functional links from hippocampus to auditory cortex.

The influence of hippocampus on auditory processing elsewhere in the brain is also revealed by lesion studies in animals, and in human patient populations. For example, the neonatal ventral hippocampal lesion rat, a widely used model for studying neurodevelopmental aspects of schizophrenia, shows altered responses to sound in inferior colliculus and auditory cortex compared to control rats, such as reduced power of the 40-Hz auditory steady state response (ASSR) (Li et al., 2018; Macedo et al., 2010; Vohs et al., 2012, 2010, 2009). In addition, the magnitude of the M100 evoked magnetic response to pure tones is diminished on the side of hippocampal sclerosis in medial temporal lobe epilepsy patients (Chatani et al., 2016; Matsubara et al., 2018), who also have abnormally lateralized ASSRs (Matsubara et al., 2018; Shigeto, 2021). Finally, pharmacological and chemogenetic shutdown of projections from dorsal CA1 via medial entorhinal cortex affects the amplitude and latency of the mismatch negativity response in mouse auditory cortex (Yi et al., 2022). See Supplementary Tables A and B for other relevant physiological studies.

In sum, multiple pathways are available for auditory information to reach the hippocampus, and for the 
hippocampus in turn to influence activity at canonical auditory structures. Although the most direct of these have been anatomically verified only in rodents to date, some electrophysiological studies hint at their presence in humans.

\section{Sound responses in the absence of a task}

Sounds that hold no particular meaning for a passively listening animal elicit a number of forms of hippocampal response (see Figure 3 for a selection and Supplementary Table $\mathrm{C}$ for a more complete list). The prominent theta component of hippocampal EEG activity has been variously associated with exploratory movement, memory encoding and retrieval, and arousal, but since the earliest hippocampal recordings in anaesthetized rabbits and cats (Green and Arduini, 1954; Jung and Kornmüller, 1938) as well as in awake animals (Eidelberg et al., 1959; Irmiš et al., 1970), increases in theta power have also been observed in response to meaningless sensory stimuli such as clicks (Figure 3A). Pure tone presentation can also reset the phase of ongoing hippocampal oscillations (Başar et al., 1979a, 1979b; Başar and Demiralp, 1995; Demiralp et al., 1996; Figure 3B) in the absence of any task. Such resets may contribute to deflections in evoked hippocampal local field potentials that have been described in anaesthetized or passively listening animals (Başar and Özesmi, 1972; Başar and Ungan, 1973; Brankačk and Buzsáki, 1986; Green and Adey, 1956; Hall and Borbely, 1970; Liberson and Cadilhac, 1953; O'Connor et al., 1992; Ungiadze, 1967) and humans (Rosburg et al., 2007).

Different classes of hippocampal cells can be distinguished based on anatomy and physiology, including the relationship of their firing to ongoing hippocampal theta oscillations (Ranck, 1973). Inhibitory interneurons tend to fire with a consistent theta phase at a rate that can increase or decrease in response to meaningless sound (Miller and Freedman, 1995; Vinogradova, 2001). Such responses have been imaged at the level of individual synaptic boutons in mouse CA1 that receive GABAergic projections from the septum (Kaifosh et al., 2013; Figure 3C). In contrast, principal pyramidal cells show occasional burst firing with no fixed relationship to theta phase. Although their most famous behavioral correlate is physical location ("place cells", O'Keefe and Dostrovsky, 1971; see Section 8), hippocampal pyramidal cells also respond to pure tones, artificial vowels, and noise, even in the absence of a task (Miller and Freedman, 1995; Vinnik et al., 2012).

Hippocampal responses to sound can be brief, persist throughout or beyond the stimulus, or be phasically modulated by its temporal structure (Lidsky et al., 1974; Martorell et al., 2019; Figure 3D). Although responses in hippocampus typically follow those recorded from auditory cortex, this is not always the case (Green and Adey, 1956; L. Zhang et al., 2018; Figure 3E). This indicates that subcortical hippocampal afferents described earlier may convey the presence of sound, a proposition further supported by the fact that responses of a subpopulation of medial septal neurons to behaviorally irrelevant sounds precede periods of elevated hippocampal and theta and gamma power (Zhang et al., 2011) and that medial septal inactivation reduces auditory responses in some hippocampal subfields (Xiao et al., 2018). The medial septal pathway may be particularly responsive to highintensity sounds in mice (Abe et al., 2014b; Kaifosh et al., 2013). Given that temporal windows of integration increase along the lemniscal pathway to primary auditory cortex and thence through the cortical hierarchy (Baumann et al., 2015; Dheerendra et al., 2021; Joris et al., 2004; Nourski et al., 2009), the alternate subcortical routes may also be those that carry rapid temporal modulations to hippocampus (Arnal et al., 2019; Chan et al., 2021; Martorell et al., 2019). 
FIGURE 3 NOT AVAILABLE IN PREPRINT SEE FIGURE LEGEND FOR REFERENCES 
Fig. 3. (Previous page). Exemplar hippocampal responses to sound in the absence of a task. (A) Bilateral LFP responses in rabbit hippocampus to a whistle [Fig. 11a in Green \& Arduini (1954)]. (B) Representative LFP traces from mouse CA1 filtered at 5-10 Hz (top) with corresponding phase (middle) and Rayleigh's test for circular uniformity (bottom) as a function of time relative to onset of a 30-ms 4-kHz pure tone [Fig. 2 in Abe et al. (2014a)]. (C) Left: Postsynaptic CA1 interneurons expressing tdTomato (red) and septo-hippocampal GABA axons expressing GCaMP5 (green) in mouse hippocampus with six labelled boutons. Right: Stimulus-triggered $\mathrm{Ca}^{2+}$ averages (+/- SEM) at the same six boutons in response to air-puffs or a $20-\mathrm{s} 10-\mathrm{kHz}$ tone. Scale bars show $50 \%$ $\Delta F / F$ and $3 \mathrm{~s}$ [Fig. 2 in Kaifosh et al. (2013)]. (D) Intervals between firing rate peaks in 338 CA1 cells in 5 mice during $40 \mathrm{~Hz}$ click stimulation (blue), random-interval click stimulation (orange) and no stimulation (gray) [Fig. 1 in Martorell et al. (2019)]. (E) Similar-latency LFP responses in cat ventral hippocampus and auditory cortex. Responses to two successive clicks separated by $15 \mathrm{~s}$ are shown side by side. [Fig. 1 in Green \& Adey (1956)]. (F) Spike rasters for a single cell in bat CA1 show selective responses to frequency sweeps of 1-ms duration (left) but not 5-ms duration (right) duration presented at $0 \mathrm{~ms}$ [Fig. 4a in Yu \& Moss (2022). (G) Mean firing rate (+/SEM) of single cell in monkey hippocampus in response to voices and other sounds [Fig. 2 in Sliwa et al. (2014)]. (H) Spike trains of rabbit CA3 neurons. Top: Activatory response at Unit A to 5th presentation of a $900 \mathrm{~Hz}$ tone. Middle/Bottom: Responses at Unit B to 5th and 8th presentations of an $800 \mathrm{~Hz}$ tone, showing suppression that habituates over trials [Fig. 1 in Vinogradova (1975a)]. (I) Grand average evoked LFP response from CA3 in 12 rat hippocampi to pairs of clicks presented $500 \mathrm{~ms}$ apart [Fig. 1 in Bickford-Wimer et al. (1990)]. (J) Grand average evoked response recorded intracranially in 21 human posterior hippocampi to clicks presented at 0 and $500 \mathrm{~ms}$ (dashed lines) [Fig. 3 in Boutros et al. (2008)]

Many of the hippocampal responses described above, whether at the single cell or evoked potential level, are not specific to particular sounds but rather scale with intensity. Because the hippocampus is involved in a wide range of cognitive and spatial processing, such relatively unselective responses are hard to attribute to auditory processing per se rather than to general arousal or orienting. However, there are exceptions to this lack of selectivity: Brown and Buchwald (1973) describe stable hippocampal tuning to tone frequency in cats, Yu and Moss (2022; Figure 3F) report duration tuning in bat CA1, and Sliwa et al. (2014; Figure 3G) find voice-selective responses in the hippocampus of monkeys - in all cases the animals were listening passively. Later sections cover selectivity during active tasks. Most single cell and evoked potential recordings during passive listening will have been insensitive to any fine-grained population coding. Calcium imaging allowing simultaneous mapping of activity of large numbers of neurons has identified robust and stable but sparse responses to passively presented odors in mouse dentate gyrus (Woods et al., 2020), but has rarely been used to detect auditory hippocampal responses.

A key factor influencing the magnitude of hippocampal responses to sound in the absence of behavior is recent stimulus history. Although habituation to a repeated auditory stimulus is a widespread neural phenomenon (Bickford et al., 1993; Miller and Freedman, 1993; Moxon et al., 1999; Picton et al., 1976), it is particularly pronounced in the hippocampus, at least for behaviorally irrelevant input in rodents and rabbits (Bickford-Wimer et al., 1990; Vinogradova, 1975b). Both firing rate changes (Vinogradova, 2001, 1975b; Vinogradova et al., 1970; Figure 3H) and evoked response deflections (Bickford-Wimer et al., 1990; Ehlers et al., 1994; Kaneko et al., 1993; Ruusuvirta et al., 1996; Figure 3I) reduce in magnitude with each repetition. In these studies, a striking reduction in sound responses can be seen after only one sound. Many rodent studies have focused on the neurochemical basis of the reduced hippocampal response to the second in a pair of stimuli separated by a 500-ms interval, because impairment of such "sensory gating" at the scalp is associated with various human psychiatric disorders (for a review see Cromwell et al., 2008). An intact hippocampus is also important for pre-pulse inhibition of the acoustic startle response - an important behavioral correlate of sensory gating (Inta et al., 2014; Kemble and Ison, 1971; see also Supplementary Table H). But habituation effects can also extend for much longer than the intervals in these gating studies - for several seconds in rats (Mays and Best, 1975) or even minutes in rabbits (Vinogradova et al., 1970). 
Selective habituation to identical stimuli naturally gives rise to a neural indicator of the presence of novelty. In many species, any perceptible change in stimulus after repetition can be sufficient to restore a large hippocampal response, with a greater effect for larger differences and more rarely occurring sounds (Başar-Eroglu et al., 1991; Csépe et al., 1989; Ruusuvirta et al., 2013; Vinogradova, 2001). Although differences between passively heard repetitive auditory standards and deviants have been detected in human hippocampus (Fuhrer et al., 2021; Herdener et al., 2010; Rosburg et al., 2007; Zevin and McCandliss, 2005; Figure 3J) these can diminish over the course of the course of an experimental session, possibly reflecting that at the level of hippocampal processing deviant sounds become less unexpected (Rosburg et al., 2007). Gross hippocampal novelty responses during passive listening are unlikely to directly contribute to the human mismatch negativity response recorded at the scalp, which has predominantly superior temporal and inferior frontal generators (Näätänen, 1990). However, the amplitude and latency of the mismatch negativity in mouse auditory cortex have recently been found to depend on a circuit via entorhinal cortex and the hippocampal trisynaptic loop (Yi et al., 2022). Hippocampal contributions to processing rare stimuli in the case of active target detection are discussed in Section 5. In the context of vision, Kumaran and Maguire (2007) identified that the novelty not of an individual stimulus but rather of associations between stimuli is particularly important in driving hippocampal activity. We will come to the role of hippocampus in representing or forming associations between sounds and other sounds, images, and locations in subsequent sections. But first we consider how this structure is involved when animals learn to associate a sound with value in appetitive or aversive conditioning.

\section{Activity during conditioning to sound}

Training animals to associate a particular sound (conditioned stimulus) with a subsequent reward or punishment (unconditioned stimulus) elicits changes in hippocampal activity over the course of learning (see Supplementary Table D). Different hippocampal synapses are modified in strength through long term potentiation (for a review see Gruart et al., 2015), leading to changes in firing rates that come to differentiate sounds that have been associated with value from those that have not (Berger et al., 1976; Disterhoft and Segal, 1978; Klee et al., 2021; Olds and Hirano, 1969). Such differential responses can arise between tones of different frequencies, or between more complex sounds such as artificial vowels with different formant structure (Itskov et al., 2012). Conditioning correlates are observed not only in firing rates of hippocampal cells, but also in local field potentials. For example, in rat dentate gyrus the amplitude of a late sustained component increases as the animal learns the association between a tone and water reward (Deadwyler et al., 1985). This contrasts with an earlier component, the magnitude of which depends on the identity of multiple preceding stimuli (whether reinforced or not), consistent with habituation effects described in Section 3. Theta activity also accompanies different phases of learning (Adey et al., 1960; Berry and Seager, 2001; Grastyán et al., 1959; Hoffmann et al., 2015) and can itself affect the success of conditioning (Berry \& Seager, 2001; Hoffmann et al., 2015).

The temporal pattern of firing that develops during conditioning may reveal something of what is learned. During eyeblink conditioning, animals learn to associate a tone with a subsequent air-puff (Berger et al., 1976). As the animal begins to show acquisition of the conditioned response - blinking just prior to the air-puff - the timecourse of hippocampal pyramidal cell firing comes to resemble the motor response, occurring progressively earlier than it as conditioning progresses (Berger et al., 1980). However, hippocampal responses and motor behavior do not always correspond; during extinction or learning of new associations, firing patterns in some units may reflect 
stimulus contingencies that are not shown in behavior (Berger and Thompson, 1982; Hoehler and Thompson, 1979; Laroche et al., 1987). Nor does the appearance of hippocampal firing require there to be an overt conditioned movement (Hirano and Yamaguchi, 1985). Although most hippocampal recordings during conditioning with auditory stimuli have been made in rats and rabbits, some of the principal findings have been replicated in cats (Patterson et al., 1977). Humans show increased metabolic and hemodynamic activity in regions including hippocampus during eyeblink conditioning compared to when sounds and air-puffs are presented in an unpaired fashion - this activity correlates weakly with learning (Blaxton et al., 1996; Cheng et al., 2008; Logan and Grafton, 1995).

Despite these extensive learning-related changes in neural activity, animals with hippocampal damage are not impaired in many conditioning settings (Berger and Orr, 1983, 1982; Brady et al., 1954; Brady and Hunt, 1955; Ross et al., 1984; Schwartzbaum et al., 1964; see Supplementary Table H). Multiple brain areas are involved during learning, and when a conditioned stimulus is sufficiently loud (Wu et al., 2013) and the unconditioned stimulus sufficiently close in time (Beylin et al., 2001), the essential neural circuitry lies elsewhere. For example the cerebellum is critical for eye-blink conditioning (Daum et al., 1993; McCormick et al., 1982; Thompson, 2005), the amygdala for fear conditioning (Phillips and LeDoux, 1994), and the striatum for appetitive conditioning (Cole et al., 2017). An intact hippocampus appears to be most important when there is a silent interval to be bridged between conditioned and unconditioned stimulus (Clark and Squire, 1998; Solomon et al., 1986; see Supplementary Table H), as well as when conditioned responses are to be limited or constrained, such as by spatial context or complex configurations of cues - especially if such configurations are to be learned incidentally and rapidly (Rudy, 2009). Subsequent sections elaborate some of these points and build on them to detail the role of hippocampus in temporal, sequential and spatial aspects of auditory processing. First, we consider some further aspects of hippocampal responses to sounds that have been associated with value or become pertinent to a task.

\section{Activity during task-based listening}

Once sounds have acquired behavioral relevance, hippocampal activity can be examined as animals perform tasks relating to them (see Supplementary Table E). For example, in rodents trained to press a lever when they detect rare frequency deviants in a train of standard sounds, late (250-500 ms post onset) differences in local field potentials to targets versus standards are particularly pronounced (Brankačk et al., 1996; Ehlers et al., 1994; Hattori et al., 2010; Shin, 2011; Shinba, 1999; Shinba et al., 1996). Other signatures of target detection include induced theta and gamma power increases (Shin, 2011) as well as more complex firing patterns in pyramidal cells than arise during standard tones (Gao et al., 2010). In one study, late firing increases occurred for targets but not standards, regardless of target intensity. This was in contrast to an earlier ( $\sim 40 \mathrm{~ms})$ peak that occurred for all tones and scaled with intensity (Shinba, 1999) and the dominance of stimulus intensity as a determinant of firing rate in the passive listening studies described earlier. For some units in the study by Shinba (1999), the degree of late firing activity correlated with both the amplitude of the late LFP response and how quickly behavioral responses were made.

In human subjects, behavioral relevance can be instilled in the absence of explicit reward through task instructions, such as to count particular target sounds or to make certain judgments. By having subjects report target counts at the end of a trial or block, explicit motor confounds are removed. In contrast to the passive listening case in 
humans described earlier, active detection of rare frequency (Altafullah et al., 1986; Halgren et al., 1995, 1980; Kropotov et al., 2000, 1995; McCarthy et al., 1989; Smith et al., 1990, 1986; Stapleton et al., 1987) or intensity (Velasco et al., 1986) targets generates large hippocampal LFP deflections with peaks between 260-500 ms. These are also accompanied by changes in local unit activity (Halgren et al., 1980; Heit et al., 1990) and may contribute to the P3/P300 scalp component (Fonken et al., 2019; Sutton et al., 1965). Some fMRI studies also show increased hippocampal BOLD for rare target sounds (Crottaz-Herbette et al., 2005; Yoshiura et al., 1999) or when listening to changes in pitch rather than identifying particular pitches (Schwenzer and Mathiak, 2011).

Even if a subject is not aware of what distinguishes a particular sound from others, previous exposure in the same experimental session can result in the formation of distinct hippocampal representations that are revealed during subsequent active listening. For example, Kumar et al. (2014) found exemplar-specific multivoxel patterns in hippocampal BOLD activity for complex noise patterns during a repetition detection task. Although subjects had been exposed to these specific exemplars multiple times during an earlier training session, they did not typically recognize their recurrence across the experiment. It may be that some degree of pre-exposure is important for hippocampal representations of sounds to form: in another study with no such pre-exposure, Liang et al. (2013) were unable to decode particular environmental sounds or spoken words from multivoxel patterns in hippocampus during target detection, even though these classes of stimuli could be distinguished from other classes (such as faces and visual words) in such patterns. Other cases of hippocampal responses during actively attended sounds will be discussed in later sections dedicated to space, sequences, and the special cases of speech and music. But first we consider the involvement of the hippocampus in temporal aspects of sound, including during the silence between a behaviorally relevant sound and the reward, punishment, or task that follows.

\section{Time and working memory for sound}

As mentioned earlier, whereas animals with hippocampal lesions are able to learn the association between a conditioned stimulus and unconditioned stimulus if these are presented in an overlapping or abutting fashion ("delay conditioning"), the insertion of a silent interval between the two ("trace conditioning") renders these animals impaired. In rodents, persistent firing of individual hippocampal neurons over the trace interval is rare (Gilmartin and McEchron, 2005; McEchron et al., 2003; McEchron and Disterhoft, 1997; Weiss et al., 1996). However, calcium imaging reveals subsets of mouse CA1 neurons that together selectively encode the conditioned stimulus identity as they span this interval (Ahmed et al., 2020; Modi et al., 2014).

This bridging of a silent interval by assemblies of hippocampal cells, each with its own temporal firing field, is not limited to conditioning paradigms in which a sound is to be associated with a subsequent appetitive or aversive stimulus. Hippocampal "time cells" (Manns et al., 2007; Pastalkova et al., 2008) are also active when animals have to retain stimulus-specific information in memory over a short silent interval, for example to compare an odor to a probe in a delayed-match-to-sample task (MacDonald et al., 2013). The population of cells encodes the retained stimulus with a fidelity that predicts subsequent task performance. Time cells have also been identified in human hippocampus during a free recall task (Umbach et al., 2020) as have cells selective for particular visual stimuli that fire at fixed phases of low-frequency $(1-7 \mathrm{~Hz})$ oscillations when they are held in mind over a delay period (Kamiński et al., 2017; Kornblith et al., 2017). Comparable findings linking unit activity to oscillatory phase or particular timepoints are yet to be reported for auditory memoranda, however the degree of synchrony 
among groups of neurons in rat CA1 can distinguish a tone frequency held in memory from another, even when such information is not carried in the firing rate of individual neurons (Takahashi and Sakurai, 2009). In humans, increases in hippocampal BOLD activity (Kumar et al., 2016) and low frequency oscillatory power (Kumar et al., 2021) emerge when human subjects keep a tone frequency in mind for comparison to a probe. It has been argued that the hippocampus is involved in retention over a few seconds only when the stimuli require complex highresolution binding (Yonelinas, 2013) or additional demands are placed on working memory (Jeneson and Squire, 2011), but neither were the case in the Kumar et al. (2021) study.

Does the presence of activity in these cases reflect a critical role for hippocampus in maintaining sound features over a temporal interval, outside of a conditioning setting? Lesioning the fimbria-fornix input to rat hippocampus impairs their ability to remember the presentation rate of click trains, as well as their duration (which is consistently underestimated; Meck et al., 1984). Medial temporal lesions in monkeys can impair their short-term retention of sound identity although it has been argued that this is an artefact of damage during surgery to auditoryprefrontal cortical pathways (Fritz et al., 2005). Dogs with medial temporal lobe lesions can retain sound identities for over a minute (Kowalska et al., 2001), although their memory for tone locations over a 10-s delay is impaired (Kowalska, 1999). Human patients with hippocampal damage can struggle to hold sounds in mind for several seconds, at least if the material cannot be rehearsed sub-vocally (Cave and Squire, 1992; Chao and Knight, 1995; Keane et al., 1995; Milner, 1972; Milner and Teuber, 1968; Penfield and Milner, 1958; Squire et al., 2001; Stefanacci et al., 2000; Wickelgren, 1968). It seems that the criticality of the hippocampus depends on species and the auditory feature to be maintained (see Supplementary Tables H, I). The relative contributions of prefrontal cortex and hippocampus to working memory remain a matter of intense study (Jin and Maren, 2015; Sreenivasan and D'Esposito, 2019; Tang et al., 2021).

That time can be considered the fourth dimension in the hippocampus has recently been extensively appraised by Banquet et al. (2021). With respect to sound, and separate from supporting holding stimuli in mind over some time interval, neurons in rodent hippocampus can be tuned to specific durations (e.g. 2 vs. 8 seconds) when relevant to auditory behavior (McEchron et al., 2003; Onoda et al., 2003). However, duration may be subordinate to pitch in hippocampal coding. Sakurai (2002) trained rats to perform both pitch and duration discrimination on the same stimulus set. They found that some hippocampal neurons were tuned to pitch alone and others to both pitch and duration, but none to duration alone. In contrast to the task-based findings in rodents, some neurons in bat CA1 are tuned to the duration (e.g. $1 \mathrm{vs} 3 \mathrm{~ms}$ ) of frequency sweeps, even during passive listening (Yu and Moss, 2022). Longer time intervals related to auditory content can also be read out from hippocampus. fMRI patterns in human hippocampus and surrounding medial temporal lobe structures associated with clips from different times in a spoken narrative are correlated with the perceived elapsed time between them (Lositsky et al., 2016). This is consistent with the idea that the medial temporal lobe provides a slowly shifting mental context that acts as a temporal tag for memories of items and episodes (Howard et al., 2005; Yonelinas et al., 2019; see also Section 13). At the other end of the temporal scale, while processing of sub-second intervals between sounds draws largely on extra-hippocampal structures, such as cerebellum and striatum (Nani et al., 2019; Teki and Griffiths, 2016), there is some indirect evidence for hippocampal involvement at these shorter timescales (see Supplementary Table I). Patients with medial temporal lobe epilepsy including hippocampal sclerosis have problems identifying patterns of durations of hundreds of milliseconds (Han et al., 2011), making anisochrony judgments on the order of tens of milliseconds (Lavasani et al., 2013), and detecting gaps in noise at below $10 \mathrm{~ms}$ 
(Ehrlé, 2001; Rabelo et al., 2015). Replicating these findings in other groups with circumscribed hippocampal damage will be valuable. As we shall see in Section 9, the hippocampus may be critically important when it comes to storing the order of stimuli in time.

\section{Sound context}

The hippocampus plays an important role in learning the constraints and contexts under which particular reward contingencies and behaviors should apply (see Table Supplementary H). Animals with hippocampal lesions fail to inhibit responses to uninformative or salient but unconditioned sounds (Freeman et al., 1973; Loechner and Weisz, 1987; Micco and Schwartz, 1971; Niki, 1967; Rickert et al., 1979, 1978; Solomon, 1977; Solomon and Moore, 1975; Swanson and Isaacson, 1967) and take much longer to learn to extinguish a conditioned response to sound than control animals (Berger and Orr, 1983, 1982; Schmaltz and Theios, 1972). Neocortical sites including perirhinal cortex are also able to support the learning of conditional dependencies, but only with sufficient exposure and when those associations are important for current behavior. The hippocampus learns more rapidly - even after a single exposure - and importantly can associate stable background elements that occur together into a context, even if they are not associated with reward or punishment at the time (Rudy, 2009; Rudy and O'Reilly, 2001). Once a context representation has been formed, pattern completion mechanisms supported by auto-associative networks in CA3 may allow a full context to be retrieved from a partial cue (Marr, 1971; McNaughton and Morris, 1987; O'Reilly and McClelland, 1994). The neocortical and hippocampal learning systems likely operate in parallel (Hebscher et al., 2019; McClelland and O'Reilly, 1995), but the dominance of the hippocampal system is illustrated by impaired context retrieval when hippocampal lesions are made after but not before learning (Lehmann et al., 2009). A broader definition of context is adopted in the contextual binding theory of human episodic memory (Yonelinas et al., 2019; see also Section 13). It holds that not only background elements of the physical environment, but also slowly changing cognitive state or mood as well as temporal context, are bound together in the hippocampus with items and events as they are encountered or experienced. The theory accounts for a range of experimental data, including interference in memory between items encountered in similar places or times, or under similar behavioural states.

The dependence of context learning on hippocampus is relevant for sound processing in a number of ways. First, hippocampally lesioned rats fail to show context-specific expression or extinction of auditory fear conditioning instead the conditioned stimulus can trigger the fear behavior even in inappropriate contexts (Corcoran and Maren, 2001; Holt and Maren, 1999; Hunsaker and Kesner, 2008). Second, a context may itself include auditory elements. For example, Grau-Perales et al. (2019) investigated the role of hippocampus in contextual control of habituation of taste neophobia, whereby mice are initially reluctant to drink novel-tasting solutions but become less averse over days. Whereas a change in the auditory context (the presence of a pure tone versus white noise) resets this attenuation in control animals, this is not the case for lesioned animals. Another relevant finding is that sounds forming part of a context can also be presented as a partial cue to reactivate hippocampus-dependent memories for the purpose of interfering with reconsolidation through inhibiting protein synthesis in rats (Ressler et al., 2021). This may be relevant for future clinical interventions relying on indirect retrieval of traumatic memories. Whether the hippocampus is involved in combining purely auditory cues into a context has not been investigated. It would be informative to establish whether hippocampally lesioned animals can automatically form a context representation on the basis of - for example - the level of reverberation and type of background noise in an 
environment, to constrain conditioned behavior.

\section{Interactions between sound and space}

The automatic learning of associations in the absence of reward, highlighted with respect to context learning in the previous section, is at the heart of the "cognitive map" concept originally proposed by Tolman (1948). He observed that rats learn the structure of a maze - subsequently enabling them to retrieve alternative routes - even when such ("latent") learning is not driven by immediate reward. Hippocampal cells that are selectively active during the exploration of space ("place cells") discovered by O'Keefe \& Dostrovsky (1971) offered a biological basis for such a map. We outline a few key features of place cells before discussing their interaction with sound.

Place cells with adjoining place fields fire in sequence as an animal traverses an environment. The formation of place fields is driven by path integration, a computation that transforms motion into a sense of location supplemented with landmark perception (Savelli and Knierim, 2019). These processes depend on cells that track head direction, speed, boundaries and other environmental properties (Lever et al., 2009; O'Keefe and Burgess, 1996; Taube et al., 1990). "Grid cells" in entorhinal cortex fire at multiple locations arranged in a hexagonal grid, tiling the environment between them (Hafting et al., 2005). Grid spacing increases from dorsal/posterior to ventral/anterior entorhinal cortex (Brun et al., 2008) but generally remains fixed across environments (Fyhn et al., 2007); these properties enable grid cells to provide a stable coordinate and metric system. There is a systematic change in the phase of theta oscillations at which a place cell fires as the animal moves through its firing field ("phase precession"; O'Keefe and Recce, 1993 in rodents; Qasim et al., 2021 in humans). This results in each theta cycle containing a representation of the recent past (places visited), present (current location) and future (planned trajectory). Such sequences are further compressed during "sharp-wave ripple" events (Buzsáki, 2015; Buzsáki et al., 1983). In rodents these happen during pauses in navigation, and can be decoded as reflecting both recently experienced ("replay") and future ("preplay") trajectories (Diba and Buzsáki, 2007). They can occur forward or in reverse, and may reflect credit assignment and planning (Foster and Wilson, 2006; Pfeiffer and Foster, 2013); see also Section 13 for a role of sharp-wave ripples in memory consolidation during sleep. Place cells can remap - that is change their firing rate or even firing field - in the presence of changes of context whether physical, such as a new cage, or task-related (Anderson and Jeffery, 2003; Jackson and Redish, 2007; Leutgeb et al., 2004; Wills et al., 2005).

Sound and place cells interact in a variety of ways. Changes that induce remapping include the association of a particular place with auditory fear conditioning (Donzis et al., 2013; Moita et al., 2004), and exposure to highintensity sound (Goble et al., 2009). Although path integration and use of visual landmarks dominate in most species, sound can also provide the basis for the formation of a spatial map and place-cell coding during echolocation in the bat (Ulanovsky and Moss, 2011, 2008, 2007). The learning of associations between locations and behaviorally-salient ultrasonic signals is facilitated by synaptic change in rat CA1 (Dietz and ManahanVaughan, 2017), under similar processes that occur for visual cues. However, even the introduction of a behaviorally-irrelevant sound can influence aspects of spatial coding, such as the relationship between locomotion speed and hippocampal theta (Long et al., 2014). In rodents, hippocampus is critical for incidental learning of the association between a sound and its location, and for auditory fear conditioning that is specific to the spatial or spatiotemporal context (Iordanova et al., 2009; Talk et al., 2002). Another case in which the absence of 
hippocampus prevents incidental learning of associations is provided by Talk et al. (2016). Hippocampallylesioned rats failed to incidentally learn the association between a noise stimulus and a particular location, demonstrated by their failure to avoid the location in the absence of the sound, after that sound had been paired with a shock. This was despite their learning to associate the sound with the shock, and control animals avoiding the location in the absence of the sound.

Space, or spatial context, has a strong influence over coding of sound in the hippocampus. For example, the firing rate of hippocampal units in response to a conditioned noise stimulus is gated by location-specific tuning (Moita et al., 2003). In another experiment some units in rat CA1 fired selectively in response to one of several rewarded artificial vowel sounds regardless of location, but this mapping only persisted as long as the spatial environment was fixed (Itskov et al., 2012). Some monkey hippocampal neurons are selective for particular types of sound (e.g. human voice over pure tone), but respond only when sounds come from a particular direction, typically behind the animal and out of its visual field (Tamura et al., 1992, 1990). Eichenbaum and colleagues have demonstrated the mixed selectivity of hippocampal neurons more generally (Eichenbaum, 2017). Representational similarity analysis over populations of neurons reveals multiplexed coding of context, location, reward, and object - often in that order of precedence (McKenzie et al., 2014). Although position-related firing is normally present from the outset, the extent to which object and reward information are encoded increases based on their relevance to behavior (Lee and Kim, 2010; Muzzio et al., 2009).

A few lesion and neuropsychological studies point to a role of hippocampus in supporting perception or memory of auditory space (see Supplementary Tables H, I). Neuropsychological cases indicate that holding the location versus the identity of a sound in mind depends to a greater extent on left and right hippocampus respectively (Lancelot et al., 2005, 2003). Dogs with hippocampal damage are also impaired at retaining sound locations (Kowalska, 1999). In humans, hippocampal lesions - particularly when coupled with superior temporal lobe damage - are associated with under-estimation of the length of sound trajectories in space (Kotelenko et al., 2013). This is somewhat consistent with the attenuation of boundary extension in visual space in patients with hippocampal amnesia (Mullally et al., 2012), and with the underestimation of temporal extent in rats with diminished hippocampal input (Meck et al., 1984).

\section{Auditory sequences and predictions}

Phase precession during spatial navigation provides for a hippocampal code of recently visited, current, and to be visited locations within a theta cycle (O'Keefe and Recce, 1993). In their firing rates, CA1 cells can code sequences of non-spatial events, such as the presentation of different odors to rats (Terada et al., 2017) or images to humans (Reddy et al., 2021). However, the breadth of applicability of a phase code to non-spatial stimuli remains to be established. In particular, unlike visual stimuli, sounds are events that do not produce fixed landmarks. However, such theta phase coding would provide a mechanism both for maintaining a representation of recent auditory past, and for predicting (for perception) or planning (for production) future sounds, for example in the context of a sentence or melody.

The hippocampus is certainly involved in auditory sequence learning in humans, including when the sounds are encountered incidentally. Patients with hippocampal lesions show severe impairment in learning probabilistic 
relationships between successive pure tones and syllables (Covington et al., 2018; Schapiro et al., 2014), mirroring results in vision (Schapiro et al., 2012). In an fMRI study, healthy subjects were exposed to tone sequence regularities during a learning phase in which they performed an orthogonal sensorimotor task (Jablonowski et al., 2018). During a subsequent test phase, they had to decide whether the next tone in a sequence would be higher or lower. Despite subjects having no explicit knowledge of the underlying regularities, accuracy was high and correlated positively with bilateral hippocampal activity during the learning phase. In an MEG study healthy subjects were presented with tones in rapid succession while engaged in an irrelevant visual task. A slow potential shift localized to hippocampus (as well as auditory and inferior frontal cortex) occurred from the point at which repetitive structure (recurring frequency patterns) occurred in a subset of sequences (Barascud et al., 2016).

In addition to these univariate markers of increased activity during sequence learning, representations of specific sound sequences emerge in hippocampus through exposure. In one fMRI experiment, multivariate patterns of activity in left hippocampus came to encode the identity of ordered sequences of spoken letters that repeated over the experiment, even though the individual elements were shared across all sequences (Kalm et al., 2013). Another study exposed subjects to continuous syllable streams, in some of which particular triplets always occurred in the same order (Henin et al., 2021). While the subjects' task was to detect the repetition of individual syllables, they implicitly learned the hidden regularities. This was reflected not only in faster reaction times during the structured sequences, but also in patterns of activity in hippocampus that became more similar over time for syllables belonging to the same triplet.

Some have argued that such sequences of sensory content (or spatial paths) become associated with pre-existing hippocampal cell assemblies that fire in a particular order, with the sensory elements themselves represented in neocortex (Dragoi and Tonegawa, 2013; Friston and Buzsáki, 2016). Related is the idea of the hippocampus as a predictive (not merely spatial) map (Gershman, 2018; Stachenfeld et al., 2017) that encodes successor representations, namely predictions of future states (discounted future occupancy) given an animal's current state (Dayan, 1993; Momennejad, 2020). These representations are thought to allow for learning of relational structure - in physical space or otherwise - separate from sensory content or reward contingency, facilitating generalization across environments that share the same relational structure (Geerts et al., 2020; Whittington et al., 2020). A large range of hippocampal findings can be accounted for in this predictive framework, including the modulation of place-cell firing fields by reward locations (Hollup et al., 2001) and barriers (Alvernhe et al., 2011; Muller and Kubie, 1987), and the asymmetric form of place fields during motion along a linear track (Mehta et al., 2000).

Indirect evidence that the hippocampus predicts future auditory content comes from responses to violation of learned rules about sound sequences. Such violations can be considered a form of associative novelty, described in the visual modality by Kumaran and Maguire (2007) and contrasting with the simple stimulus novelty covered at the end of Section 3. Violations of auditory-sequence order elicit scalp components in EEG (Takakura et al., 2003) and MEG (Recasens et al., 2018) that have been localized to hippocampus. In the latter these were accompanied by greater hippocampal theta power and phase locking. In probabilistic sound sequences, violation is not all-or-nothing. Instead, time-varying continuous measures of uncertainty (entropy) and surprise can be derived based on learned statistics. Cheung et al. (2019) trained a Markov model on harmonic progressions in pop songs and compared its estimates of uncertainty and surprise during novel progressions to BOLD activity. Anterior hippocampus (along with amygdala and auditory cortex) reflected the interaction between these factors, being 
elevated when chords deviated substantially from strong expectations, or when they met relatively imprecise ones. Interestingly, these were the same conditions that elicited the greatest pleasure ratings in listeners. Other fMRI work has been more equivocal as to whether hippocampus tracks uncertainty in auditory sequences (Tobia et al., 2012), and in another study the hippocampal BOLD signal was reduced in tone sequences in which simple or hierarchical rules concerning pitch and duration were violated compared to when they were met (Martins et al., 2020). Disparate findings may relate to functional heterogeneity of hippocampal fields, or position of activity along the long axis.

Violation and surprise responses in hippocampus are consistent with it acting as a comparator, with predictions passed from CA3 to CA1 where they are combined with sensory input from entorhinal cortex (Hasselmo and Wyble, 1997; Lisman and Grace, 2005; Vinogradova, 2001, 1975b). However, there is also evidence for hippocampus sending predictions to sensory cortex. In the study by Recasens et al. (2018), predictable sequences elicited elevated effective connectivity (based on alpha-band Granger causality) from right hippocampus to Heschl's gyrus, compared to unpredictable sequences. In other work with perfectly predictable intervals of sound and silence, a spatial independent component of the BOLD signal that included hippocampus led the auditory cortex signal (Langers and Melcher, 2011).

In a more specific and naturalistic demonstration of information exchange between hippocampus and sensory cortex, Michelmann et al. (2021) presented spoken stories to intracranially-implanted epilepsy patients twice in succession. Auditory cortical sites were identified at which content-specific high-gamma activity in the second run emerged earlier than that in the first, based on Granger causality. At peaks of this predictive measure, mutual information between this activity and (mostly $<35 \mathrm{~Hz}$ ) activity in hippocampus was maximal, with hippocampus leading cortical activity by $270 \mathrm{~ms}$. Section 2 outlined pathways along which such predictions could be conveyed. Any resulting error signals could be passed in the reverse direction to update corresponding hippocampal models (Barron et al., 2020).

A missing piece in the puzzle is evidence of specific predicted auditory content being decodable in hippocampus. A number of studies have decoded visual content that is predicted on the basis of simple auditory cues from hippocampal BOLD patterns (Aitken and Kok, 2021; Kok et al., 2020; Kok and Turk-Browne, 2018). Predicted visual content can also be decoded from hippocampus when that prediction is triggered by auditory cues on the basis of semantic knowledge. In human intracranial recordings, when spoken words primed a particular semantically-related image, high gamma hippocampal activity that was more similar across periods prior to and during the image predicted faster response times (Jafarpour et al., 2017). Furthermore, such activity showed similarity structure across stimuli that reflected the similarity of the predicted objects in semantic space. Another study with a similar task found the most pronounced hippocampal theta activity during sentences and words that set a strong semantic context for the subsequently presented picture (Piai et al., 2016). It remains to be investigated whether sounds predicted on the basis of associations learned in the short-term (such as the next note in a melody introduced in an experiment) or through semantic context (such as the sound of a bark following a picture of a dog) can be decoded from hippocampal activity before they are heard.

\section{Navigating frequency space}


We have seen that rodent grid cells can provide a basis for spatial navigation (Hafting et al., 2005). In humans, there is evidence that entorhinal grid cells support navigation in virtual (Doeller et al., 2010; Jacobs et al., 2013), imagined (Bellmund et al., 2016) and visual (Julian et al., 2018; Killian et al., 2012; Meister and Buffalo, 2018; Nau et al., 2018) space, as well as time (Ezzyat and Davachi, 2014). Other dimensions of experience can be represented in a similar way; these include social hierarchies spanned by affiliation and power (Tavares et al., 2015), a two dimensional space of body part lengths (Constantinescu et al., 2016), an imagined two dimensional odor space (Bao et al., 2019), and semantic spaces of written words (Solomon et al., 2019). Most of these studies indirectly measure the presence of grid cells by virtue of hexadirectional symmetry of the BOLD response with respect to navigation direction. In some cases, a neural correlate of distance has also been identified in abstract spaces. The evidence is growing that computational circuitry in the hippocampus and entorhinal cortex can facilitate "navigation" through and memory of any arbitrary space.

An important auditory example has been described by Aronov et al. (2017), who trained rats to depress a lever while a tone increased in frequency and to release it in a target frequency range for a reward. They identified CA1 cells that had particular frequency firing fields during this task and others that fired preferentially at the start or end of a trial. The number of tuned cells decreased when the animal was no longer responsible for releasing the lever but was still rewarded when the tone reached the target frequency. When the tone changed in a block without involvement of the animal and in the absence of reward, no such tuning existed. During the active task, cells in the entorhinal cortex could have multiple firing fields. Notably, some of these same hippocampal and entorhinal cells also had place and grid fields when the animal was instead foraging in an open arena. Of all CA1 cells recorded, approximately a quarter had auditory and place fields, a half one or the other, and a quarter neither. This example is different from those described earlier in which discrete individual sounds have acquired behavioral significance (e.g. conditioning studies, Sakurai 2002) - here the continuous range of presented frequencies is represented. Another important point is that the trial duration and the rate at which the tone frequency changed was varied throughout the experiment. Units retained their frequency tuning across this variability, meaning that they were not simply tuned to the absolute time elapsed in a trial. However subsequent research has established that ensembles of CA1 and entorhinal cells in rats and humans can carry temporal information on a range of scales and individual time cells can stretch their tuning in accordance with the demands of a task (Mau et al., 2018; Reddy et al., 2021; Shimbo et al., 2021; Tsao et al., 2018). It is therefore possible that the units in Aronov et al. (2017) reflected relative time in task ("retime cells" in MacDonald et al., 2011) or relative "distance" to the target sound. The work of Aronov et al. therefore raises intriguing possibilities about the representation of an acoustic dimension in hippocampus during an active task that require critical reappraisal in further experiments.

\section{Auditory objects and scenes}

Sections 9 and 10 showed that the hippocampus tracks sequences of sound and may map one of its most salient dimensions, frequency. In Section 3 we gave examples of intensity and amplitude modulation rate affecting hippocampal responses and also described how changes in sound features can drive hippocampal responses through release from habituation. In cluttered acoustic scenes, the rate of change of sound features is among the key determinants of which sequential elements should be grouped into auditory streams or objects (Bregman, 1990). An auditory perceptual object, like its visual counterparts, is defined by the binding of multiple sensory features, represents a source distinct from others in the scene, and is invariant over different sensory instances 
(Bizley and Cohen, 2013; Griffiths and Warren, 2004; Kubovy and Van Valkenburg, 2001). The medial temporal lobe is important in visual object perception, and the hippocampus in particular supports perception and construction of visual scenes - particular configurations in space (Barense et al., 2009; Chadwick et al., 2013; Hassabis et al., 2007; Lee et al., 2012; McCormick et al., 2021; Mullally et al., 2012; Zeidman et al., 2015).

Does the hippocampus contribute to the formation of auditory objects or their collections into scenes? Much of the abstraction of different auditory features and their combination into object representations is accomplished in the ascending subcortical auditory pathway and auditory cortex. For example, adaptation occurs in the auditory nerve for grouping of harmonics by common onset (Holmes and Roberts, 2011), tuning for combinations of auditory features is present in primary auditory cortex (Bizley et al., 2009), and representations in auditory association cortex demonstrate object-level intensity gain control (Simon, 2015) and correlate with object-level perception (Billig et al., 2018) and attention (O'Sullivan et al., 2019). The extraction of timbre, defined by combinations of features other than intensity and pitch, involves higher auditory-associated cortex including planum temporale and anterior superior temporal sulcus (Kumar et al., 2007; Warren et al., 2005), and illusory percepts of vowels based on apparent object continuity have correlates in superior temporal sulcus and middle temporal gyrus (Heinrich et al., 2008).

Unlike in vision (Devlin and Price, 2007), evidence for medial temporal involvement in auditory object formation, perception, and scene analysis is scarce (Bizley and Cohen, 2013; Christison-Lagay et al., 2015; Griffiths and Warren, 2004; Snyder and Elhilali, 2017). Although patients with Alzheimer's disease show impairment in auditory segregation and scene analysis tasks, structural and functional correlates of these deficits are reported in lateral temporal and parietal cortices, rather than in the medial temporal lobe (Golden et al., 2015; Goll et al., 2012). However there is some support for the number of perceptual objects in a simple acoustic scene being tracked there. Direct human intracranial recordings found hippocampus to be among those structures where activity distinguished between perceptual interpretations of bistable tone triplets that could be heard as one or two streams (Curtu et al., 2019). Another human study found a medial temporal source for a late P400-like scalp potential associated with successful detection of a mistuned harmonic in a tone complex, which gives rise to the percept of two concurrent auditory objects (Alain et al., 2001). In rodents, damage to perirhinal cortex (hippocampus was not tested) impaired rats in binding discontinuous temporal vocalization elements into an object to act as a conditioned stimulus in fear conditioning (Bang and Brown, 2009) and we have already described involvement of hippocampus in bridging temporal gaps, both during auditory working memory and trace conditioning, and in representing auditory sequences in memory. Less direct evidence for hippocampal involvement in auditory scene segregation is its elevated activity in subjects performing a verbal working memory task in noise compared to in quiet (Manan et al., 2012).

Two important ingredients to successful parsing of an auditory scene are the ability to distinguish an object of interest from the background, and to restore a partially masked sound on the basis of prior knowledge. These requirements to "separate" and "complete" auditory representations under different circumstances bring to mind two terms describing particular computations in support of memory, thought to involve hippocampus (Marr, 1971; McNaughton and Morris, 1987; Rolls, 2013). Pattern separation refers to the storing of distinct activity patterns for memories that share similar features. Modelling suggests that the large number of granule cells in dentate gyrus can support the sparse coding necessary to transform the overlapping representations from entorhinal cortex 
and project the result to $\mathrm{CA} 3$ and beyond for storage and consolidation. In the case of an unfolding and spectrotemporally overlapping acoustic scene, might this separation act rapidly enough for the results to be read out during online perception? The hippocampus can certainly guide perceptual sampling of a cluttered visual scene (Kragel et al., 2021) and the same may be true of audition.

The other concept, pattern completion, refers to the retrieval of a memory on the basis of a partial cue, thought to be supported by auto-associative or attractor networks in CA3 (Rolls, 2013). Such a completion process could potentially retrieve features of known sources (e.g. the vocal characteristics of a known conversation partner) or anticipate likely continuations of interrupted sentences based on semantic knowledge or prior exposure. In this vein, one study found theta synchrony between medial temporal lobe (only parahippocampal gyrus was available for analysis) and auditory cortex during the illusory continuation of familiar music when interrupted by noise (Müller et al., 2013). The passing of predictions from hippocampus to auditory cortex based on learned sequences and discussed in Section 9 would also constitute a form of pattern completion. These ideas remain mostly speculative, and a critical role for hippocampus in auditory object formation has not been demonstrated. Alternatively, it is possible that hippocampus is only required when incorporating auditory elements into scenes primarily determined by visual objects defined in a spatial framework. In the next section we consider auditoryvisual and other crossmodal interactions in hippocampus.

\section{Multi- and supra-modal representations and associations}

The intrinsic circuitry and external connectivity of the hippocampus allow it to bind sensory experience across modalities. Patients with hippocampal lesions have impaired recognition memory for associations between simultaneously presented faces and voices (Mayes et al., 2004; Vargha-Khadem et al., 1997), and between other sounds and scenes (Mayes et al., 2004) or abstract images (Borders et al., 2017). At the same time, transcranial magnetic stimulation of parietal sites identified on an individual subject level to be functionally connected to hippocampus boosts memory for word-face pairs (Wang et al., 2014). Hippocampal BOLD activity during an object encoding period scales with the number of features to be integrated, with location making a greater contribution than color or sound (Cooper and Ritchey, 2020). Activity is elevated for successful encoding or retrieval of cross-modal associations compared to within-modality pairs (Butler and James, 2011; Gottlieb et al., 2010; Joassin et al., 2011; Persson et al., 2011) and demonstrates functional connectivity with cortical sites including superior temporal gyrus during such multimodal associations (Cooper and Ritchey, 2019; Griffiths and Fuentemilla, 2019; Joassin et al., 2011; Love et al., 2011). In an experiment involving memory for text-sound associations, pairings with the greatest hippocampal activity at encoding were recalled most accurately and showed the most similar neocortical patterns across encoding and retrieval (Danker et al., 2016). In these experiments, learning the association was explicitly required as part of the task. However, elevated hippocampal BOLD has also been demonstrated when images are combined with emotionally congruent music compared to when they are presented alone during an emotion-rating task without an explicit memory component (Baumgartner et al., 2006).

Tone frequency can combine with a non-auditory dimension to define a semantic space that is represented in the medial temporal lobe. Viganò \& Piazza (2020) trained participants to associate particular quadrants in a twodimensional space of visual shape and tone frequency with four different non-word labels. After training, right 
entorhinal cortex showed tuning to direction of navigation through this space during a one-back task that used both the audiovisual objects and the written semantic labels. There was also distance tuning in this region, but this was weaker than that in medial prefrontal cortex.

Auditory stimuli can also trigger or be subsumed into super-modal conceptual representations in the hippocampus. For example, Quiroga and colleagues have identified human hippocampal units that respond selectively to famous people, whether in photograph form, or as a written or spoken name (Quiroga, 2020; Quiroga et al., 2009). Twice as many neurons respond to the image than to the sound in these studies, and while there are neurons that respond to the image but not the sound, the reverse is not true (however this bias may be a result of the distribution of stimuli used). To establish the extent to which auditory representations are subordinate in the hippocampus it would be valuable to establish whether such concept tuning can be identified based on multiple auditory instances only, such as the spoken word "dog" and the sound of a dog barking, or a person's spoken name and their voice.

\section{Auditory elements of episodic memories and their consolidation}

The hippocampus not only binds across sensory modalities, but situates these multimodal objects in a spatiotemporal context to form memories of particular episode (Gelbard-Sagiv et al., 2008; Paz et al., 2010). We note the apparent contradiction that under its proposed role in statistical learning the hippocampus generalizes to learn the probabilities of transitions between sounds over multiple presentations, but it is also able to form discrete memories for individual episodes. Modelling work suggests that statistical learning could rely more on the monosynaptic connection between entorhinal cortex and CA1, where inhibition and sparsity are less pronounced, than along the trisynaptic pathway from entorhinal cortex through dentate gyrus and CA3, likely important for memory of individual episodes (Schapiro et al., 2016).

The extent and nature of the hippocampus' ongoing involvement in maintaining and retrieving episodic memories has been controversial. The standard consolidation model (Squire and Zola-Morgan, 1991; Squire and Alvarez, 1995) holds that episodic memories that initially depend on hippocampus to index distributed content in neocortex (Teyler and DiScenna, 1986) become consolidated over time, with direct links between those components strengthening, and hippocampal dependence declining. Such a model accounts for the graded retrograde memory deficit observed in amnesic patients such as H.M., whereby older memories are relatively preserved - however, it has been argued that these are of more of a semantic nature than vividly episodic. Alternative accounts - such as multiple trace, trace transformation, and contextual binding theories - propose that hippocampus continues to be involved, either in indexing or reconstructing distributed cortical content, or maintaining such content itself (Nadel et al., 2000; Winocur and Moscovitch, 2011; Yonelinas et al., 2019).

Sound-related evidence for ongoing hippocampal involvement in supporting rich memories (rather than memories that are given a semantic label), comes from findings that BOLD activity there scales with the vividness of a retrieved episodic memory, including its auditory content (Sekeres et al., 2018). Furthermore, patients with medial temporal lobe damage report fewer perceptual (including auditory) details when retrieving episodic memories than controls (St-Laurent et al., 2014). Hippocampal BOLD continues to be present when hearing a recording of one's own autobiographical memories (Svoboda and Levine, 2009) or a melody previously associated with a particular object and location (Prabhakar et al., 2018) after several days. When listening to familiar music, subjects 
show greater hippocampal BOLD activity as they retrieve specific autobiographical episodes associated with the music than more general ongoing events or personal knowledge from the relevant period in their lives (Ford et al., 2011). Hippocampus is also more active during such retrieval than when attending to structural features of the music (Kubit and Janata, 2018).

Which memories get consolidated in humans during sleep can be biased by presentation of relevant sounds. In one study, subjects learned object-location pairs while presented with an object-specific sound, then slept during a scanning session (van Dongen et al., 2012). Greater hippocampal activity when previously heard sounds were repeated during sleep was associated with better retention of object-location pairs as tested the following day. In another study, participants learned motor patterns that were associated with different tones. Presentation of one of those tones during sleep led to faster execution of the cued compared to the uncued pattern the following day, with a corresponding difference in bilateral hippocampal activity and hippocampal connectivity to motor areas (Cousins et al., 2016).

In rodents, hippocampal involvement in consolidation of auditory memories during sleep was demonstrated by Bendor \& Wilson (2012), who first paired spatial trajectories with auditory cues in behaving rats. Presenting one of these sounds during subsequent sleep increased the probability that the place cell sequence encoding the related trajectory would be reactivated. In another study, auditory cortical patterns that occurred when rats approached a location associated with a sound were recapitulated during sleep, both spontaneously and when cued by the auditory stimulus (Rothschild et al., 2017). This activity predicted subsequent hippocampal sharp-wave ripples, which in turn predicted subsequent auditory cortical activity, suggesting a bidirectional exchange of information. Content-specific sharp-wave ripple playback from hippocampus to sensory cortex has also been detected in humans, but so far only during awake visual recall (Norman et al., 2019). The fact that auditory signals, unlike visual information, can trigger hippocampal activity during sleep points to possible overnight learning applications (Harrington and Cairney, 2021), discussed further in Section 18.

\section{Perception and memory of speech and music}

Speech (Supplementary Tables F, J) and music (Supplementary Tables G, K) are two classes of sound particularly important in human communication. Intelligible speech elicits greater hippocampal activity than unintelligible speech, regardless of whether that greater intelligibility is due to acoustic clarity or provision of prior information (Clos et al., 2014; Davis et al., 2011; Davis and Johnsrude, 2003). In challenging listening conditions, speech understanding is positively correlated with the distinctness of candidate word representations in left hippocampus and superior temporal sulcus (Blank et al., 2018). The hippocampus is also important in resolving syntactic or semantic ambiguity based on information from earlier in a sentence or exchange (Kurczek et al., 2013; Rubin et al., 2011). Another possible role of hippocampus during conversation is in monitoring one's own speech, possibly through comparing predictions of motor commands with resulting auditory feedback (van de Ven et al., 2020; see also Rummell et al., 2016 for self-generated sounds in mice).

Beyond online speech perception there is substantial neuropsychological evidence for a key role of left hippocampus in particular for the learning and recall of verbal material (Barbeau et al., 2005; Boon et al., 2011; Cavazzuti et al., 1980; Coras et al., 2014; Dulay et al., 2004; Frisk and Milner, 1990; Gadian et al., 2000; Goldstein 
et al., 1988; Helmstaedter et al., 1997; Helmstaedter and Elger, 1996; Huijgen et al., 2015; Jayakar et al., 2015; McMillan et al., 1987; Meyer and Yates, 1955; Mueller et al., 2012; O’Brien et al., 2003; Rausch and Crandall, 1982; Squire et al., 2001; Tachibana et al., 1999; Vargha-Khadem et al., 1997; Witt et al., 2014). The separation of language and spatial function in left and right hippocampi respectively is sufficiently robust that it is routintely adopted in clinical work, including neurosurgical planning. Neuroimaging also supports this dichotomy, with left hippocampal BOLD signal during exposure to novel pseudo-words greater than that to pseudo-words that had also been heard the previous day (consistent with a novelty response), but also correlating across subjects with subsequent recall (Davis et al., 2009). The novelty of not only a word but also its category can drive hippocampal activity during encoding (Dolan and Fletcher, 1997). Other studies have shown greater hippocampal activity immediately prior to or during successful compared to unsuccessful word learning (Park and Rugg, 2009; Petersson et al., 1999; Urgolites et al., 2020), as well as when listening to short stories about which questions are to be asked, compared to pure tones (Schmithorst et al., 2006). Another study found hippocampal activity returning to baseline more slowly than other areas in a verbal working memory task (Kato et al., 1998). Connectivity between left hippocampus and left lateral temporal regions during successful (declarative) encoding has also been demonstrated in BOLD timeseries (Gagnepain et al., 2011) and in theta/gamma phase coherence in intracranial recordings (Babiloni et al., 2009). Encoding of spoken information into hippocampal memory may occur predominantly at perceived event boundaries, a result based on Granger causality analysis of human intracranial data during naturalistic story listening (Michelmann et al., 2021). This is consistent with work implicating the hippocampus in segmenting ongoing experience, such as when it marks boundaries between scenes in movies (Baldassano et al., 2017; Ben-Yakov and Henson, 2018).

While the above studies point to a role for hippocampus in the encoding of memory for words, deficits in consolidation have been identified in groups with presumed hippocampal damage. Patients with transient epileptic amnesia (Hoefeijzers et al., 2013) and with presymptomatic autosomal dominant Alzheimer's disease (Weston et al., 2018) show accelerated long-term forgetting, such that word memory is impaired after a week, but not after 30 minutes. This suggests that verbal acquisition itself may not be impaired when hippocampus is compromised, but rather the durability of the memories that form.

Whereas verbal memory deficits are associated particularly with left hippocampal damage, memory for melody may depend to a greater extent on right-hemisphere structures. However, although right hemisphere damage has been associated with selective impaired recognition for melody versus lyrics (Samson and Zatorre, 1992, 1991), and a reduced "mere exposure" effect where previously heard melodies are usually judged as more likeable than novel ones (Samson and Peretz, 2005), lesions in these studies extended to extra-hippocampal regions in the temporal lobes, known to be important in musical perceptual and discrimination (Milner, 1962; Samson and Zatorre, 1994; Zatorre, 1984 but see Koike and Ishijima, 1996). Indeed, a number of neuropsychological case studies indicate that intact hippocampi are not necessary for a range of musical perception abilities relating to timing and pitch (Esfahani-Bayerl et al., 2019), nor for learning of new music (Cavaco et al., 2012; Valtonen et al., 2014) or even recognition memory for unfamiliar melodies and memory abilities (Finke et al., 2012). These findings are consistent with intact musical memory in patients with Alzheimer's disease, in whom other hippocampal-dependent memories are impaired (Baird and Samson, 2009; Cuddy et al., 2015).

However, as we have seen in earlier sections, the hippocampus not being critical for a task does not prevent it 
from tracking relevant stimulus or behavioral variables. For example, during listening to a rich naturalistic stimulus in the absence of a task, left hippocampal BOLD activity was associated with repeated occurrences of musical motifs after regressing out acoustic predictors (Burunat et al., 2014), and showed functional connectivity with regions involved in holding melodies in mind, including dorsolateral prefrontal cortex, cerebellum, and the supplementary motor area. Schmithorst (2005) also identified melody-specific activity in hippocampus using an independent components analysis of fMRI data during passive listening, and in a study of memory for newlylearned melodies retrieval success was associated with greater right hippocampal BOLD signal (Watanabe et al., 2008). As with speech there is electrophysiological evidence for greater hippocampal processing at musical phrase boundaries (Knösche et al., 2005).

Hippocampal activity and connectivity has been detected during a range of tasks during music listening, including tone detection (Lehne et al., 2014), timbre and tonality deviant detection (Janata, 2002), temporal order judgments (Mueller et al., 2015), spontaneity judgments (Engel and Keller, 2011), and memory encoding (Bonetti et al., 2021). Hippocampus is also activated during passive music listening, compared to a silent or scrambled baseline (Brown et al., 2004; Mueller et al., 2015, 2011; Mutschler et al., 2010). We will see in Sections 15 and 16 that familiarity and emotional aspects of music may partly drive these responses.

\section{Long-term familiarity}

Sections 4 and 5 covered responses to sounds that had recently acquired behavioral relevance, for example through aversive conditioning or prior exposure in a target detection task. Longer-term familiarity with an auditory stimulus can also affect the magnitude of the hippocampal activity it drives. The hippocampus of rabbits, cats, and monkeys can show greater responses to familiar sounds, such as hisses, than to louder but unfamiliar synthetic sounds, such as tones and clicks (Grastyán et al., 1959; Green and Arduini, 1954; Tamura et al., 1990). Note that greater responses to familiar than unfamiliar sounds contrast with the novelty responses described in Section 3, which presumably arise through a different mechanism operating over a shorter timescale.

While in the above studies not only the familiarity but also the gross acoustical features of the contrasted sounds differed, other work has attempted to control the latter. For example, when children listened to their mother's voice, fMRI connectivity across hippocampus, reward- and voice-sensitive regions was greater than when listening to other female voices (Abrams et al., 2016). Long-term familiarity with verbal expressions correlated with right posterior hippocampal BOLD activity; this was not the case for musical melodies presented to the same subjects (Gagnepain et al., 2017; Groussard et al., 2010b). There are other indications that the hippocampus is relatively unimportant in familiarity processing of music. Using multivoxel pattern analysis, long-term familiar songs could be distinguished from songs heard on the same day or novel songs in anterior cingulate and presupplementary motor area, but not in the medial temporal lobe (Jacobsen et al., 2015). Subjects listening to their favorite song showed less auditory-hippocampus connectivity than when listening to other songs in the same genre (Wilkins et al., 2014). Additionally, a study of patients with medial temporal lobe resections were impaired in verbal learning and recall but had a spared feeling of familiarity when hearing short excerpts of well-known music (Huijgen et al., 2015).

These negative findings are consistent with visual work identifying a greater dependence on hippocampus (and 
connected diencephalic structures) for explicit recollection, compared to general familiarity thought to be supported more by parahippocampal/perirhinal circuits (Aggleton and Brown, 2006; Brown and Aggleton, 2001; Tsivilis et al., 2008). However, for audition the story is mixed, with some studies finding greater responses in hippocampus for familiar compared to unfamiliar music (Pereira et al., 2011; Plailly et al., 2007) and deficits in recognition of well-known songs in patients with hippocampal damage (Papp et al., 2014). In addition, it is not always straightforward to isolate processes relating to familiarity from those involved in explicit recollection, as familiar sounds can elicit the recollection of particular events.

\section{Emotion and sound}

Also hard to dissociate from sound familiarity are sound preference and other emotional drivers of hippocampal activity, both due to "mere exposure" effects of repetition (Hunter et al., 2010; Samson and Peretz, 2005) and the fact that people choose to listen to music they enjoy. In some experiments that show greater hippocampal BOLD activity for familiar than unfamiliar music, stimulus manipulations affect both long-term familiarity and pleasantness ratings (Koelsch et al., 2006; Mueller et al., 2015, 2011; Pereira et al., 2011). Particularly intense emotional responses to music can be evoked by familiar music that listeners select for themselves. Blood and Zatorre (2001) reported reduced blood flow in left hippocampus during listening to self-selected music experienced as intensely pleasurable music, eliciting "chills" and other physiological responses, in comparison to control music selected by other subjects. This reduced response is thought to arise through inhibitory projections from the nucleus accumbens, part of the dopaminergic reward system in which activity increases during chills. These two regions demonstrate increased BOLD functional connectivity during music to which listeners assign increasing value - even new music with which they are not familiar (Salimpoor et al., 2013).

Medial temporal sites - and the focus is more parahippocampal gyrus than hippocampus proper - are involved in processing emotion in music, beyond the familiarity effects described above. Lesion (Gosselin, 2006) and neuroimaging (Blood et al., 1999; Ferri et al., 2014) data link the rating of dissonant harmonies as unpleasant to parahippocampal gyrus, paralleling work with unpleasant images (Lane et al., 1997). Diffuse medial temporal lesions affect more general estimates of pleasantness and arousal in music (Gosselin et al., 2005; Khalfa et al., 2008) and impair recognition of musical emotion (Dellacherie et al., 2008; Gosselin et al., 2011, 2005; Omar et al., 2011; Papp et al., 2014) (but see Dellacherie et al., 2011), and posterior hippocampal grey matter volume is greater in frontotemporal dementia patients with musicophilia, (an abnormal craving for and delight in music) than in those without (Fletcher et al., 2013).

Not only simple musical emotional contrasts (such as happy versus sad, joyful versus fearful) but also subtler differences involving arousal or valence alone modulate (para)hippocampal BOLD activity and its relationship with that in auditory and reward networks (Flores-Gutiérrez et al., 2007; Koelsch et al., 2018, 2013; Koelsch and Skouras, 2014; Trost et al., 2012), and individuals with high alexithymia scores show less such modulation (Koelsch et al., 2007). Some of these effects may arise through the uncertainty and surprise associated with harmonic progressions, which jointly predict pleasantness ratings and the BOLD response in regions including hippocampus as described previously (Cheung et al., 2019).

Section 12 considered hippocampal correlates of multimodal stimulus processing; these are also apparent with 
respect to emotionally-charged material. For example, combining emotional music and pictures elicits greater (para)hippocampal BOLD than the pictures alone (Baumgartner et al., 2006) and the presence of narrative action in a neutral film increases modulation of hippocampal activity by emotional music (Eldar et al., 2007). However, shutting off uninformative input by closing the eyes leads to more extreme valence ratings and elevated hippocampal BOLD in response to emotional music (Lerner et al., 2009). Not only the structural elements of a musical piece but also expressive performance determine its emotional profile; this too affects hippocampal activity (Chapin et al., 2010a; Engel and Keller, 2011). The involvement of hippocampus in processing emotional music occurs regardless of expertise and although it can habituate over the course of listening it does not generally require active engagement (Brown et al., 2004; Chapin et al., 2010b; Mutschler et al., 2010; Pallesen et al., 2009).

Music is not the only means of expressing emotion through sound. (Para)hippocampal areas are also sensitive to different types of laughter (Szameitat et al., 2010), as well as to prosodic (Wiethoff et al., 2008) and semantic (Bellace et al., 2012; Mitchell et al., 2003) emotion cues in speech. These sites are more sensitive to natural than to computer-generated speech (Beaucousin et al., 2006), and respond to or support processing of vocal fear, anger, happiness, surprise, disgust (Bonora et al., 2011; Fowler et al., 2006; Kotz et al., 2013; Leitman, 2010; Phillips et al., 1998; Sander et al., 2005), as well as more complex auditory emotional expression such as pride, guilt, and boredom (Alba-Ferrara et al., 2011). Several studies describe functional connectivity signatures of auditory emotion processing. For example, alarm sounds rated as highly unpleasant are associated with reduced hippocampal BOLD activity and functional connectivity consistent with suppression by the amygdala (Hirano et al., 2006). This coincides with worse encoding of a concurrent visual stimulus, consistent with findings in animal models showing that stress and fear impair learning. Subjects with misophonia, for whom everyday sounds evoke strong negative emotional responses, show elevated functional connectivity between anterior insula and a salience network that includes hippocampus (Kumar et al., 2017). Individual differences in responses to aversive sounds may also have structural correlates - in one large study, subjects' sensitivity to a noise stimulus correlated with hippocampal grey matter volume (Kliuchko et al., 2018). In another study, synthetic sounds rated as more aversive generated elevated cerebral blood flow in (para)hippocampus and amygdala (Mirz et al., 2000). Negative reactions to certain sounds may relate to the energy in the "roughness" range of $30-150 \mathrm{~Hz}$; click trains presented at this rate are rated as highly salient and aversive and bring about neuronal synchronization at the presentation rate, particularly in the hippocampus and insula (Arnal et al., 2019).

\section{Phantom percepts}

Aversive auditory experiences do not require an external stimulus. Tinnitus (see Supplementary Table L) usually takes the form of a low-intensity, high-frequency ringing or white noise that is typically readily masked by environmental sounds but can be chronic and emotionally distressing (Jastreboff, 1990). It tends to co-occur with hearing loss, and many theories of its generation are based on maladaptive change to deafferentation, or deficient noise-cancelling (Jastreboff, 1990; Noreña, 2011; Rauschecker et al., 2010; Schaette and Kempter, 2006). Indepth reviews and integrative accounts are available elsewhere (De Ridder et al., 2014; Sedley et al., 2016); here we focus on correlates in (para)hippocampus but note that comorbidity of tinnitus with hearing loss, distress, depression, cognitive dysfunction and insomnia presents a challenge in establishing specific neural signatures (Adjamian et al., 2014; Bhatt et al., 2017; Crönlein et al., 2007; Hallam et al., 2004; Hwang et al., 2009; Melcher et al., 2013). At the molecular level, damage to rat inner hair cells leads to tinnitus-like behavior only for those 
animals whose hippocampus and auditory cortex fail to mobilize Arc (Singer et al., 2013), a protein involved in long-term potentiation and adjusting synaptic transmission following sensory deprivation (Korb and Finkbeiner, 2011). Tinnitus-like behavior is also present in rats with noise-induced disruption to neurogenesis and cholinergic and GABAergic pathways in hippocampus (Kraus et al., 2010; L. Zhang et al., 2018; Zhang et al., 2021). In terms of gross structure, the volume of the left hippocampus is smaller in patients with tinnitus than in controls matched for hearing loss (Boyen et al., 2013; Landgrebe et al., 2009) and its surface area correlates negatively with tinnitus handicap inventory scores (Tae et al., 2018). Hippocampal grey matter abnormalities have been identified in tinnitus sufferers using diffusion tensor imaging (Gunbey et al., 2015), and tinnitus symptoms have been reported after hippocampus damage or resection (Corkin et al., 1997; Kreyberg et al., 1992; Paquette et al., 2017; Rey et al., 1984). Of note, it is non-trivial to distinguish correlates of tinnitus from those of impaired (especially suprathreshold) hearing loss that can co-occur.

These negative links between hippocampal structure and tinnitus stand in distinction to functional studies that often (but not always, Shulman, 1995; Shulman et al., 1995) report a positive association between hippocampal activity and tinnitus loudness or incidence (De Ridder et al., 2006; Lockwood et al., 1998). Resting state fMRI indicates greater connectivity of the hippocampus with auditory cortex and beyond for louder tinnitus percepts and longer tinnitus durations (Chen et al., 2017b; Ueyama et al., 2013), and highlights the left hippocampus as a key node in functional networks of chronic tinnitus patients compared to controls (Lan et al., 2022). In contrast, functional connectivity between hippocampus and subcortical auditory nuclei is reduced in rats with salicylateinduced tinnitus and hyperacusis symptoms (Chen et al., 2015); these animals also show elevated hippocampal local field potential responses to noise-bursts compared to controls (Chen et al., 2014). Distinct neural correlates of salicylate- and noise exposure have been described and these complicate the search for a unified signature of tinnitus (Eggermont, 2013). In humans, regional blood flow in other medial temporal structures, including amygdala and parahippocampal gyrus, also differs across suppressed versus active tinnitus states (Mirz, 2000) and levels of distress (Schecklmann et al., 2013), as well as between participants with tinnitus compared to hearing-impaired and normal-hearing controls (Carpenter-Thompson et al., 2014; Laureano et al., 2014). Indeed, resting state activity in parahippocampal gyrus more consistently differentiates these groups than does activity in hippocampus proper (Chen et al., 2017a; Song et al., 2012) and may form an important tinnitus hub (De Ridder et al., 2014; Sedley et al., 2015).

Auditory hallucinations (see Supplementary Table M) are another example of sound perception in the absence of a stimulus. These have been associated with hippocampal lesions in a number of reports, but rarely isolating the hallucinations from comorbid symptoms, for example in patients with schizophrenia (Maller et al., 2012; Suzuki et al., 2003; Takebayashi et al., 2002). A similar caveat holds for early imaging studies that reported increased medial temporal lobe activity in patients with schizophrenia featuring auditory hallucinations (DeLisi et al., 1989; Friston et al., 1992; Liddle et al., 1992; Medoff et al., 2001; Volkow et al., 1987) although some studies are more specific, with greater medial temporal rCBF for auditory compared to tactile hallucinations (Musalek et al., 1989) or with longer durations of auditory hallucination (Copolov et al., 2003). Within-subjects comparisons have revealed greater (para)hippocampal activity for periods with auditory hallucinations compared to those without (Dierks et al., 1999; Raij et al., 2009; Shergill, 2001; Shergill et al., 2000; Silbersweig et al., 1995). As with tinnitus, compared to hippocampus proper (Bentaleb et al., 2002; Jardri et al., 2009, 2007; Lennox et al., 2000; McGuire et al., 1993; Sommer et al., 2008), the involvement of parahippocampal gyrus is most reliable across 
studies (Diederen et al., 2010; Hoffman et al., 2008; Jardri et al., 2011). In both cases parahippocampal gyrus may convey aberrant predictions originating in hippocampus to auditory cortex, but the relative lack of corresponding hippocampus activity remains to be explained.

\section{Short-term effects of sound on non-auditory tasks and hippocampal activity}

We have described immediate responses to sound in the hippocampus and its possible involvement in a range of auditory tasks, functions and percepts. In Section 2 we also outlined effects of electrically or optogenetically stimulating hippocampus on auditory brain regions. Here we cover cases in which sound stimulation instigates, boosts or entrains hippocampal oscillations to affect non-auditory cognitive function. We consider oscillations over three timescales, as shown in Figure 4. First, slow $(<1 \mathrm{~Hz})$ oscillations reflect global fluctuations in cellular excitability - alternating phases of hyperpolarization and depolarization across large populations of neurons. In humans these oscillations are likely driven by prefrontal cortex but propagate via parahippocampal and entorhinal cortex to hippocampus (Nir et al., 2011) where they can synchronize ripple events (Born and Wilhelm, 2012). Introducing short bursts of pink noise at the peak of the up state of these oscillations during sleep increases their amplitude and leads to improved memory encoding the following day (Ngo et al., 2013; Figure 4A). It has not been possible to directly record from hippocampus during overnight auditory stimulation, however in one study the pink noise protocol was applied during a nap, and a picture encoding task was performed subsequently in a scanner (Ong et al., 2018). The magnitude of slow oscillation enhancement during the nap correlated with picture encoding and with hippocampal BOLD during encoding. This may indicate that boosting slow oscillations allowed previous memories to be consolidated to cortex, freeing up hippocampal resources for subsequent encoding.

The second timescale of oscillations at which auditory entrainment may boost hippocampal function is the theta range. Roberts et al. (2018) presented audiovisual stimuli at either $5.5 \mathrm{~Hz}$ or $14 \mathrm{~Hz}$, or white noise, between training and testing of verbal memory. In the $5.5-\mathrm{Hz}$ condition only, theta power recorded at the scalp was enhanced both during entrainment and subsequent retrieval, and hippocampus-dependent source memory was selectively boosted. Other studies have generated binaural beats in the theta range by presenting pure tones of frequencies differing by $5 \mathrm{~Hz}$ to separate ears, then directly measuring hippocampal activity in neurosurgical patients (Derner et al., 2021, 2020, 2018; Figure 4B). Binaural beats were associated with improved source and item memory along with increased phase synchrony and unit firing in human (para)hippocampus, with firing rate differences between monaural and binaural beat conditions correlating across subjects with differences in memory performance.

Third, $40-\mathrm{Hz}$ click trains or stimuli amplitude-modulated at this rate have long been known to elicit a strong steady state response in human auditory cortex (ASSR, Galambos et al., 1981). However they also modulate unit activity in mouse hippocampus (as well as auditory cortex and medial prefrontal cortex) such that firing tends to cluster at fixed phases of the 40-Hz cycle (Martorell et al., 2019; Figure 4C). Remarkably, click trains presented at this rate (but not with random timing) reduced signs of Alzheimer's pathology (amyloid load and tau phosphorylation) in hippocampus of this mouse model and boosted performance in recognition and spatial memory tasks. Effects were larger when the auditory stimulus was paired with visual flicker at the same rate - this combined stimulation also induced an increase in $40 \mathrm{~Hz}$ power and a clustering effect of microglia around amyloid deposits. The 
implications for dementia treatment are considerable, and work is ongoing to test the same approach in human trials (Chan et al., 2021). The mechanism is not yet understood but networks of inhibitory interneurons generate peaks at this low-gamma frequency, which may also be involved in coupling CA3 and CA1 during memory retrieval (Colgin et al., 2009; Mably and Colgin, 2018).

\section{A White noise bursts and slow $(<1 \mathrm{~Hz})$ oscillations in humans}

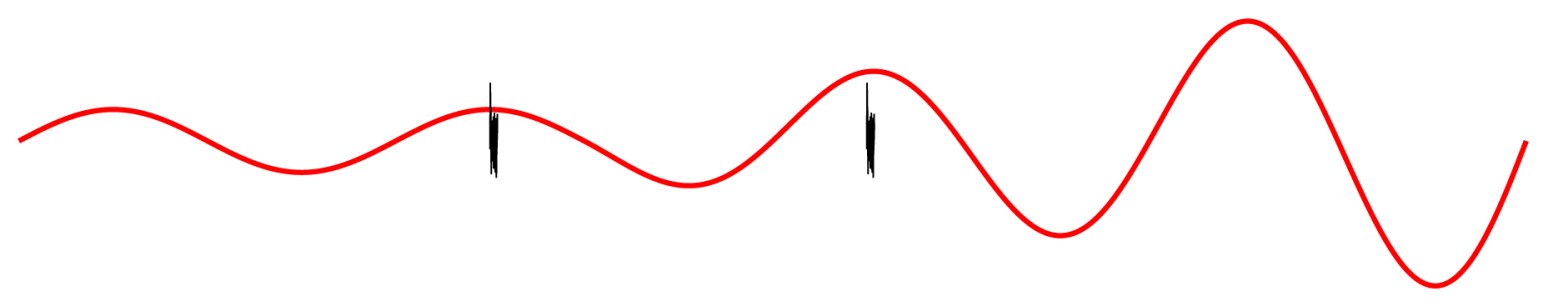

B Binaural beats and theta $(5 \mathrm{~Hz})$ oscillations in humans

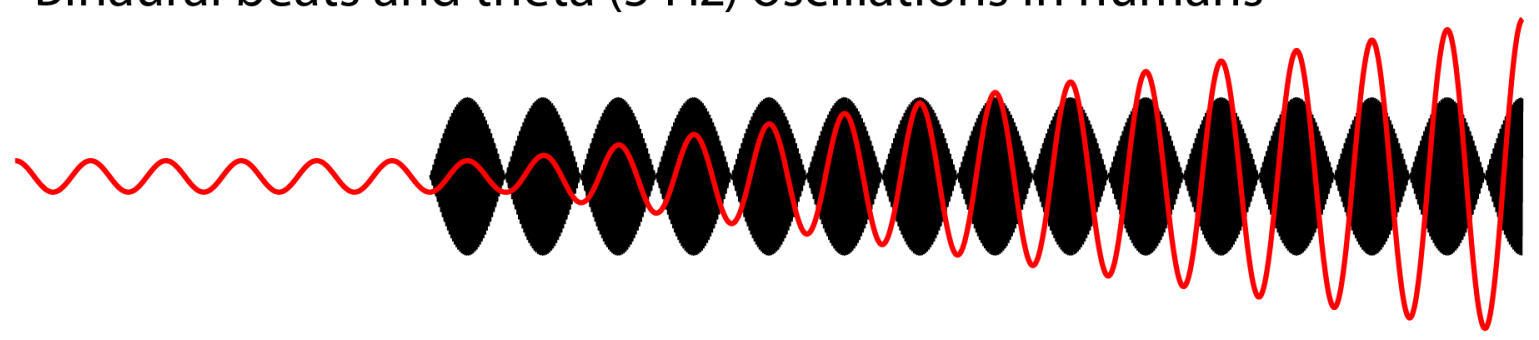

C Click trains and gamma $(40 \mathrm{~Hz})$ phase synchrony in mice

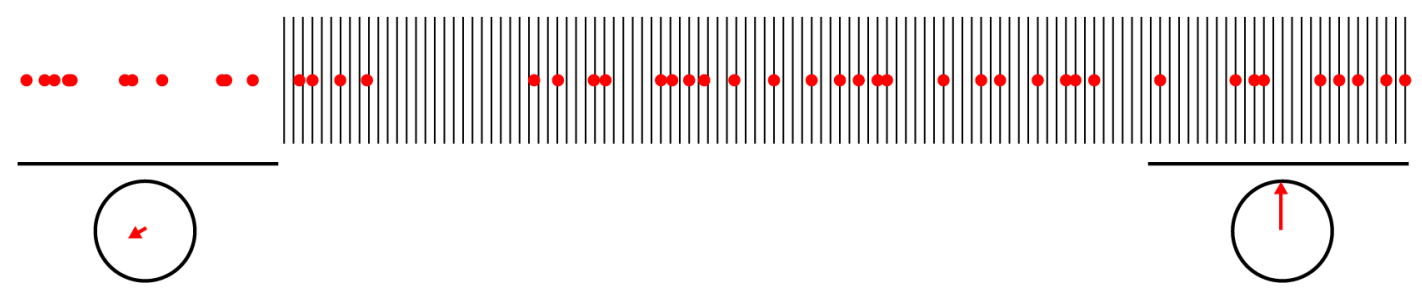

Fig. 4. Auditory entrainment of hippocampal rhythms (see Section 18 of main text for related behavioral outcomes). (A) Suitably timed white noise bursts (black) boost widespread cortical slow $(<1 \mathrm{~Hz})$ oscillations (red), which propagate to hippocampus and synchronize sharp-wave ripples (not shown) in humans [Ngo et al., 2013]. (B) Dichotically presented pure tones separated in frequency by $5 \mathrm{~Hz}$ generate binaural beats (black) and boost hippocampal theta oscillations (red) in humans [Derner et al., 2018]. (C) $40 \mathrm{~Hz}$ click trains (black) affect firing of hippocampal CA1 units (red dots), increasing phase synchrony (red arrows) at the same (gamma) frequency in mice [Martorell et al., 2019].

\section{Long-term experience with sound and its absence}

Having considered effects of short-term auditory exposure we turn now to longer-term experience with sound and its positive and negative effects on hippocampal structure and function (see Supplementary Table N and Figure 5). If the hippocampus maps out non-spatiotemporal dimensions as suggested in Section 10 then experts at navigating such dimensions might be expected to demonstrate gross anatomical differences, given the finding of Maguire et al. (2000) that London taxi drivers have enlarged posterior hippocampi. Such associations have been 
found, with positive relationships between years of training and anterior hippocampal grey matter volume in musicians (Groussard et al., 2014, 2010a) and piano-tuners (Teki et al., 2012). For spatial navigation and other hippocampus-dependent tasks, the generalizibility of a volume-function relationship to a non-expert population has not been demonstrated (Clark et al., 2020; Weisberg and Ekstrom, 2021). However with respect to music, the volume of parahippocampal gyrus (hippocampus was not tested) was found to correlate positively with an index of musical sophistication in a group of 73 older adults after controlling for intracranial volume (ChaddockHeyman et al., 2021). Longitudinal studies, similar to those run for taxi drivers (Woollett and Maguire, 2011), will be important to establish causality in any of the above relationships.

Functional differences in hippocampus of musicians and non-musicians have been identified through task-related BOLD activity and connectivity (Alluri et al., 2017, 2015; Burunat et al., 2018; Chapin et al., 2010a; Gagnepain et al., 2017) as well as in scalp EEG responses, for example to incongruous harmonic endings to phrases (James et al., 2008). These cross-sectional studies again may to some extent reflect pre-existing group differences in personality traits, socio-economic status or cognitive factors (Corrigall et al., 2013; Orsmond and Miller, 1999). In one longitudinal study, right hippocampal BOLD activity during music listening and imagery was positively correlated with success in a subsequent six-week piano training course; this marker of predisposition contrasted with cerebellar and fronto-parietal activity that increased over the course of that training (Herholz et al., 2016). In another study, tone patterns containing rhythmic deviants were presented to musicians before and after two semesters of university-level musical training, and to a control group of musicians receiving no such additional training (Herdener et al., 2010). Greater left anterior hippocampal responses to deviants occurred in the second session, only for the group receiving training. A positive relationship between musical aptitude and degree of activity in that same region was also found in cross-sectional analysis of a separate group. Taken together, the results suggest that at least some music-related differences in hippocampal function arise from training rather than innate ability.

The eye-catching finding that listening to a Mozart sonata improves spatial reasoning (Rauscher et al., 1993), an effect later established to be a rather non-specific consequence of arousal (Pietschnig et al., 2010; Thompson et al., 2001), led to interest in whether passive music exposure brings about hippocampal changes in animal models. Such exposure, especially prenatally or in development, can result in differential gene expression and regulation, elevated markers of neurogenesis, and changes in synaptic density and regulation in rodent (Angelucci et al., 2007; Chikahisa et al., 2006; Kim et al., 2006; Lee et al., 2016; Meng et al., 2009; Xing et al., 2016a) and avian (Chaudhury et al., 2010, 2008, 2006; Chaudhury and Wadhwa, 2009) hippocampus (but see Rizzolo et al., 2021). These effects are often accompanied by functional improvements, including in spatial (e.g. Xing et al., 2016a) and fear learning (e.g. Meng et al., 2009) tasks. Findings are mixed as to whether particular musical elements, such as rhythmic structure, versus more general auditory arousal, are responsible for the behavioral and neuronal changes (Angelucci et al., 2007; Chaudhury et al., 2008, 2006; Field et al., 2007; Hu et al., 2014; Kirste et al., 2015; Sanyal et al., 2013a, 2013b; Xing et al., 2016b; Yang et al., 2014b, 2014a). Understanding how and why levels of the protein brain-derived neurotrophic factor increase through music exposure and engagement may be key to this question (Brattico et al., 2021).

In contrast to positive effects of music listening, noise exposure elevates hippocampal stress hormone levels 


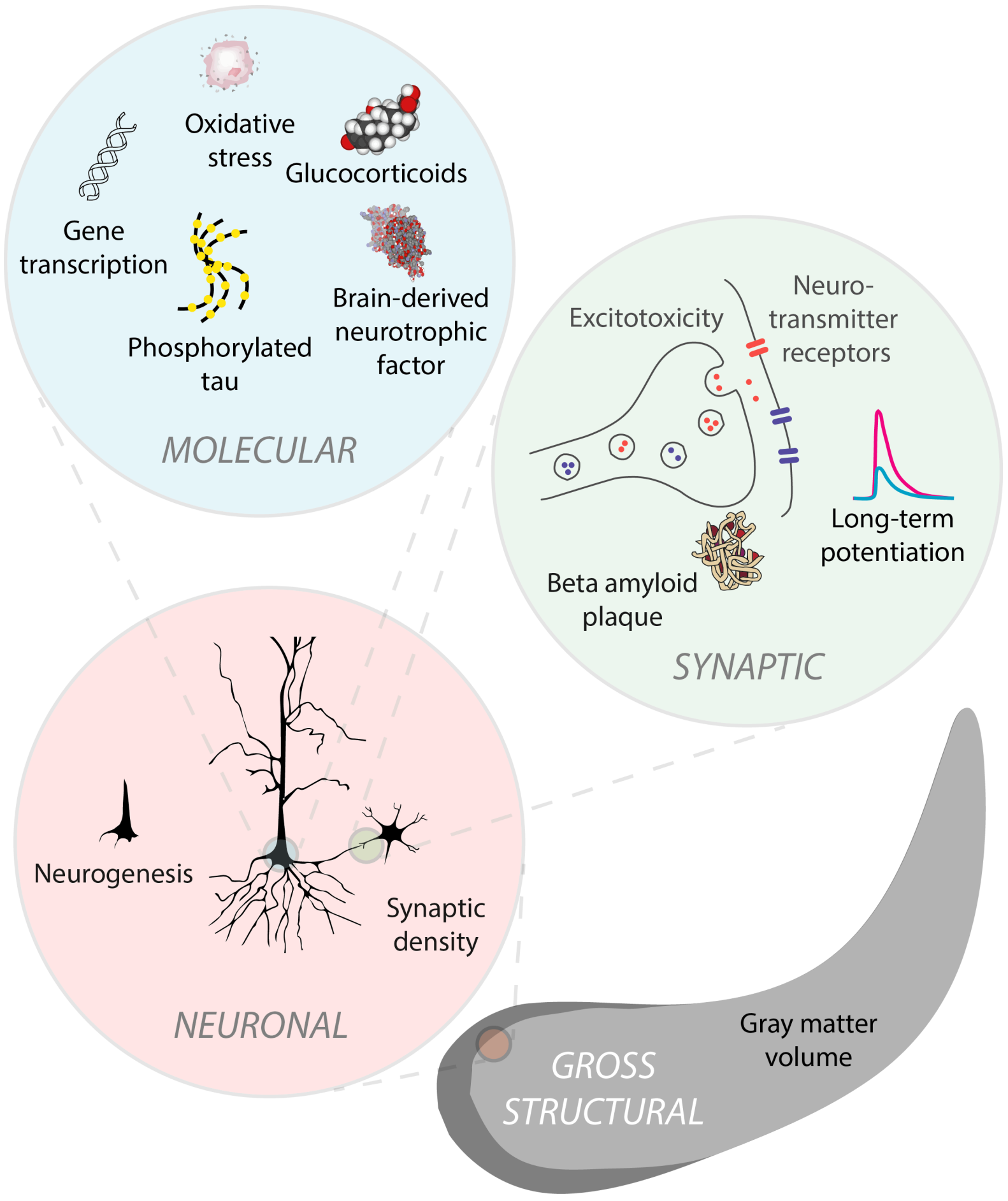

Fig. 5. Examples of processes at molecular, synaptic, neuronal and gross structural hippocampal levels on which auditory experience (such as music listening or training, noise exposure, and auditory deprivation) can act. See Section 19 of main text and Supplementary Table N for details and references. The causal pathways underlying such effects are largely yet to be established.

(Barzegar et al., 2015; Britton et al., 1992; Campeau and Watson, 1997; Ferrarese et al., 1991; Gai et al., 2017; Jáuregui-Huerta et al., 2011; Jin et al., 2017) and markers of oxidative stress (Ambrosini et al., 2005; Cheng et al., 2016, 2011; Manikandan et al., 2006; Uran et al., 2014), often resulting in accelerated cell death and reduced neurogenesis (Cui et al., 2009, 2013; Frenzilli et al., 2017; Gonzalez-Perez et al., 2011; B.-K. Kim et al., 2013; 
Kim et al., 2006; Kraus et al., 2010; Liu et al., 2010; Manikandan et al., 2006; Säljö et al., 2000, 2002; Uran et al., 2012). Noise-induced reductions in NMDA receptors and related protein expression (Cui et al., 2013, 2009; Kapolowicz and Thompson, 2016; Singer et al., 2013) impair hippocampal long term potentiation (Barzegar et al., 2015; Cunha et al., 2018, 2015; de Deus et al., 2017) and learning that depends on it (Barzegar et al., 2015; Cheng et al., 2011; Cui et al., 2009, 2012a; Cunha et al., 2015, 2018; de Deus et al., 2017, 2021; Di and Qin, 2018; Haider et al., 2012; B.-K. Kim et al., 2013; Kim et al., 2006; Manikandan et al., 2006; Uran et al., 2010, 2012, 2014). Spatial memory deficits may also result through destabilization of place cell receptive fields (Goble et al., 2009). A range of neurotransmitters and other markers of neural activity in the hippocampus are sensitive to noise exposure (Campeau and Watson, 1997; Cui et al., 2009, 2012a; de Deus et al., 2021; Di and Qin, 2018; Fernandes and File, 1993; Haider et al., 2012; Lai, 1987, 1988; Lai et al., 1989; Lai and Carino, 1990; Manikandan et al., 2006), as is DNA integrity (Frenzilli et al., 2017). Abnormalities in glial cells and levels of their activating proteins have also been described following noise presentation (Cui et al., 2015; Frenzilli et al., 2017; Huet-Bello et al., 2017) as have signatures of Alzheimer's disease pathology, including tau hyperphosphorylation (Cheng et al., 2011; Cui et al., 2015, 2013, 2012a, 2012b; Frenzilli et al., 2017; Gai et al., 2017) and elevated amyloid- $\beta$ and pro-inflammatory protein levels (Cui et al., 2015; Jafari et al., 2019).

Some of these changes depend on the duration (Barzegar et al., 2015; Cheng et al., 2011) or level (HosseiniSharifabad and Sabahi, 2008; Matt et al., 2018; Singer et al., 2013) of the noise, others can reverse over time (Cui et al., 2012a; Di and Qin, 2018; Frenzilli et al., 2017) or be protected against by various compounds (Abousetta et al., 2014; Azman et al., 2016; Li et al., 2014; Sundaramahalingam et al., 2013; S. Wang et al., 2016) or exercise (T.-W. Kim et al., 2013). Many are not specific to sound, arising due to a range of stressors (Chen et al., 2010; B.-K. Kim et al., 2013; Kim and Diamond, 2002). Given rodent work showing that rapid subcortical auditory pathways are more important for conveying noise than tone presence to hippocampus (Zhang et al., 2018), it will be important to establish whether such pathways also exist in humans to judge the clinical relevance of these findings. More in-depth reviews of the effects of noise on hippocampus are available elsewhere (Kraus and Canlon, 2012; Manukyan, 2022; Nadhimi and Llano, 2021; Zhang et al., 2022).

While noise exposure can cause deafness, most acquired hearing loss occurs gradually over the lifetime. Agerelated hearing loss (presbycusis) is an independent risk factor for dementia, estimated to account for $9 \%$ of cases (Livingston et al., 2017). The hippocampus and entorhinal cortex are among the earliest sites showing dysfunction and atrophy in Alzheimer's disease (Braak and Braak, 1991; Khan et al., 2014), and poorer hearing in midlife is associated with steeper volumetric declines in these regions later in life (Armstrong et al., 2019). A large longitudinal study also found that individuals developing a hearing loss between scans an average of two years apart showed greater decline in left hippocampal gray matter volume than those developing no such hearing loss (Fitzhugh and $\mathrm{Pa}$, 2022). This group also showed a greater decrease in functional connectivity over time between auditory cortex and hippocampus (see also Andine and Holmer (2022) for lower fucntional connectivity between superior temporal regions and hippocampus in individuals who are deaf from birth compared to controls). Connectivity in subjects with presbycusis is also disrupted between hippocampus and the inferior parietal lobule, to an extent that correlates with the degree of working memory impairment (Chen et al., 2020). The nature of any causal link between age-related hearing loss (or other listening impairments) and the hippocampal pathology and cognitive decline associated with dementia has not been fully established (Griffiths et al., 2020; Nadhimi and Llano, 2021; Tuwaig et al., 2017) but recent animal work offers some clues. The C57BL/6 mouse exhibits 
progressive hearing impairment and is widely used as a model for presbycusis. These animals, which also demonstrate impaired spatial behavior, suffer synaptic degeneration, decreased cell numbers and abnormal morphology in hippocampal CA1 and CA3 as well as altered neurotransmitter receptor expression (Beckmann et al., 2020; Dong et al., 2018; Yu et al., 2011). In studies with otherwise healthy mice, occluding ears to simulate conductive hearing loss also impairs hippocampal neurogenesis and increases microglial invasion and stress responses (Kurioka et al., 2021). Temporary conductive hearing loss can also be induced through ear drum perforation; in rats this interferes with NMDA receptor-mediated currents in CA1, reducing local field potentials and impairing spatial learning (Zhao et al., 2018). Finally, administration of ototoxic drugs to induce sensorineural hearing loss leads to hippocampal degeneration, impaired spatial learning, and tau phosphorylation (Shen et al., 2021). Whether similar effects occur in humans is not yet known.

\section{Synthesis and outstanding questions}

We have reviewed imaging, recording, lesion and neuropsychological work across species, charting diverse interactions between sound and the hippocampus. These include a hierarchy of responses, from reacting nonselectively during passive listening, through tracking associations between particular sounds and rewards, to mapping out auditory dimensions during behavior. We identified hippocampal involvement in linking sounds with each other, with stimuli in other sensory modalities, and with spatiotemporal context to form episodic memories. We also detailed roles of the hippocampus in processing music, speech, emotional sound, and aberrant auditory percepts, as well as how hippocampal structure and function can be shaped by auditory experience. We described how the machinery that supports spatial navigation and memory may be harnessed in more general sequential processing, including that relating to sound. Questions remain as to the extent to which the hippocampus helps build representations of auditory objects and scenes, which are structured in time, frequency and space. However, the synthesis of auditory-hippocampal interaction that emerges does not support an adequate explanation of hippocampal function based on spatial navigation and episodic memory. The auditory work requires an explanation based on broader aspects of perception and cognition.

What does it mean to say the hippocampus is "involved in" a function? It may not be critical for a given task, but the stimulus and behavioral information it tracks becomes available either for an optimal solution to the current problem, or as part of a more complex representation than is available upstream and/or that may be drawn on subsequently. Although single-neuron tuning to specific auditory features is most pronounced when they are behaviorally relevant (Aronov et al., 2017; Itskov et al., 2012) we have also identified plenty of cases of hippocampal responses to sound during passive listening. Determining the critical dependency of certain auditory computations and functions on the hippocampus will require a combination of optogenetic techniques in animal models to temporally shut down pathways, invasive and non-invasive stimulation in humans, and administering standardized batteries of auditory cognition (e.g. those previously applied to dementia patients, Hardy et al., 2020) to groups with particularly circumscribed hippocampal damage (e.g. autoimmune limbic encephalitis, Lad et al., 2019).

What characteristics of hippocampal circuits might lend themselves to auditory cognition? A non-exhaustive list could include: theta phase precession to track past, present and future in sound sequences; a monosynaptic pathway that can learn probabilistic contingencies between sounds and other stimuli; the means to separate 
patterns of signals arising from distinct auditory objects or in different contexts through sparseness and inhibition in dentate gyrus; the means to compare stored representations in CA3 with, or to complete, or predict from, current auditory input; connectivity with subcortical and cortical sites that provide auditory information processed with respect to distinct features or to different extents.

A number of steps can be taken to further test the idea that circuits in hippocampus and entorhinal cortex that support memory and navigation in physical space can be harnessed for auditory processing. Neuroimaging work that has indirectly found evidence for grid-cell like structures supporting non-spatial navigation (e.g. of semantic spaces) could be extended to cover auditory dimensions. Human intracranial recordings may provide more direct evidence for tuning to auditory features if coupled with the right task. Methodological advances in magnetic sensory technology, headcasts and source localization may facilitate temporally resolved magnetoencephalographic hippocampal recordings during listening (Alberto et al., 2021; Hill et al., 2020; Meyer et al., 2017; Pizzo et al., 2019; Recasens et al., 2018; Tierney et al., 2021).

An important outstanding question is the extent to which spatial coding and processing in the hippocampus dominates over other dimensions of experience. O'Keefe \& Krupic (2021) argue that cells responding selectively to non-spatial stimuli are in fact feature-in-place cells, at least in non-humans; for humans they argue the spatial cognitive map has been enhanced by language and its metaphorical use). That is, responses that appear to be determined by stimulus characteristics are in fact primarily driven by the animal's spatial location - but this is masked because animals are not typically tested in different locations. This argument finds support in the Itskov et al. (2012) result that tuning to a particular sound only held when the animal's location was fixed. Also relevant is a finding that hippocampal responses during eyeblink trace conditioning only occurred in cells in whose place field the animal was situated, with conditioning leading to an arousal-related enhancement in firing (Shan et al., 2016). However, Mount et al. (2021) identified different hippocampal populations responding during acquisition versus extinction of trace conditioning. This suggests that arousal-modulated place tuning is unlikely alone to account for selective responses to particular sounds in individual cells.

Relatedly, is the hippocampus only engaged in forming auditory scenes and objects when there is an element of spatial variation rather than when spectral and/or temporal information alone are present? Experiments are needed in which subjects have to perceive, remember or mentally construct combinations of sounds composed of different feature conjunctions (such as a sound with a high amplitude modulation rate and low carrier frequency, followed by one with the reverse configuration). Comparable experiments with visual objects by Maguire and colleagues (Dalton et al., 2018; Zeidman et al., 2015) have been important in establishing the nature of hippocampal involvement in visual scene processing.

More straightforward analogues of experiments in other sensory modalities can answer pressing questions that have already been addressed for olfaction (in rodents) and vision (in humans and non-human primates), and that might be even more relevant for audition for which stimulation always unfolds over time. For example, does the phase at which rat hippocampal neurons fire for sequential sounds precess as the sequence unfolds (c.f. Terada et al., 2017)? Can calcium imaging reveal sparse encoding of behaviorally irrelevant auditory stimuli in mouse dentate gyrus (c.f. Woods et al., 2020)? Do sharp wave ripples in human hippocampus during auditory memory tasks reflect content-specific encoding, retrieval, replay and pre-play, in partnership with cortex (c.f. Norman et 
al., 2019)? Can predicted auditory content be decoded from human hippocampus and does this depend on stimulus complexity (c.f. Kok et al., 2020)? In addition, some proposed hippocampal coding schemes, based on spatiotemporal similarity (Turk-Browne, 2019), or modality/exemplar-invariant concepts (Quiroga et al., 2009) have not yet been tested against substantial auditory data.

We have seen how rhythmically structured sound at a range of timescales provides a non-invasive means of driving or entraining neural oscillations, which hippocampus plays a role in orchestrating across cortex. There are promising signs that such auditory interventions can improve memory and even disrupt pathology in animal models of disease. Compared to electrical, magnetic and invasive stimulation, an acoustic approach is accessible, inexpensive and generally non-intrusive/intimidating. To harness its full potential it will be important to understand how the auditory pathway and hippocampus work together with other regions including prefrontal cortex in bringing about these changes. More broadly, although we have focused on the hippocampus throughout this piece, considering such a richly connected structure in isolation will have only given part of the picture whether with respect to conditioning, sound sequences, spatial and temporal aspects of sound, auditory memory, musical emotion, or phantom percepts.

If the findings from animal models concerning links between hippocampus, hearing loss, tinnitus and dementia are to translate into to clinical advances in humans it is important that we understand the degree of overlap between species in terms of anatomy, connectivity, physiology, and function. Recent results are reassuring in this regard. For example the identification of human hippocampal place cells, time cells, and phase precession - originally discovered in rodents - indicate that at least some of the coding and computational principles overlap. At the same time, post-mortem anatomical data on human auditory-hippocampal pathways is almost non-existent. We also acknowledge that different species evolved in the context of unique environmental constraints, with a range of sensory and higher-level capabilities (Basile et al., 2020). Despite these varied contexts under which the hippocampus operates, there are considerable areas of convergence in the auditory results we have reported. Another caveat is that we have surveyed literature linking sound and hippocampus as comprehensively as possible, but have not systematically reviewed each sub-topic in a meta-analytical manner, nor included every study in which no hippocampal involvement was reported. Findings should be interpreted accordingly and we hope that this review provides a launching point for further study.

In conclusion, the hippocampus receives all manner of auditory information regardless of its behavioral relevance at the time. Any tuning to acoustic features or criticality of involvement is strongest when there is a requirement to associate sounds with locations, rewards or punishments separated in time, other sounds, or stimuli in other sensory modalities - either for perception or memory. The structural, synaptic and biochemical components that facilitate such processing are themselves sensitive to the organism's auditory experience - both in the short and long-term.

\section{Acknowledgments}

This work was supported by the Wellcome Trust, United Kingdom (WT106964MA), Medical Research Council, United Kingdom (MR/T032553/1), and the National Institutes of Health, United States of America (5P50DC000242-35). 


\section{References}

Abe, R., Sakaguchi, T., Kitajo, K., Ishikawa, D., Matsumoto, N., Matsuki, N., Ikegaya, Y., 2014a. Sound-induced modulation of hippocampal $\theta$ oscillations: NeuroReport 25, 1368-1374. https://doi.org/10.1097/WNR.0000000000000274

Abe, R., Sakaguchi, T., Matsumoto, N., Matsuki, N., Ikegaya, Y., 2014b. Sound-induced hyperpolarization of hippocampal neurons: NeuroReport 25, 1013-1017. https://doi.org/10.1097/WNR.0000000000000206

Abousetta, A., Makhlouf, N.A., El-Beshbishy, R.A., 2014. The effects of concomitant Ginkgo intake on noise induced Hippocampus injury. Possible auditory clinical correlate. Egyptian Journal of Ear, Nose, Throat and Allied Sciences 15, 231-239. https://doi.org/10.1016/j.ejenta.2014.05.003

Abrams, D.A., Chen, T., Odriozola, P., Cheng, K.M., Baker, A.E., Padmanabhan, A., Ryali, S., Kochalka, J., Feinstein, C., Menon, V., 2016. Neural circuits underlying mother's voice perception predict social communication abilities in children. Proceedings of the National Academy of Sciences 113, 6295-6300. https://doi.org/10.1073/pnas.1602948113

Adey, W.R., Dunlop, C.W., Hendrix, C.E., 1960. Hippocampal Slow Waves: Distribution and Phase Relationships in the Course of Approach Learning. Archives of Neurology 3, 74. https://doi.org/10.1001/archneur.1960.00450010074007

Adjamian, P., Hall, D.A., Palmer, A.R., Allan, T.W., Langers, D.R.M., 2014. Neuroanatomical abnormalities in chronic tinnitus in the human brain. Neuroscience \& Biobehavioral Reviews 45, 119-133. https://doi.org/10.1016/j.neubiorev.2014.05.013

Aggleton, J.P., Brown, M.W., 2006. Interleaving brain systems for episodic and recognition memory. Trends in Cognitive Sciences 10, 455-463. https://doi.org/10.1016/j.tics.2006.08.003

Ahmed, M.S., Priestley, J.B., Castro, A., Stefanini, F., Solis Canales, A.S., Balough, E.M., Lavoie, E., Mazzucato, L., Fusi, S., Losonczy, A., 2020. Hippocampal Network Reorganization Underlies the Formation of a Temporal Association Memory. Neuron 107, 283-291.e6. https://doi.org/10.1016/j.neuron.2020.04.013

Aitken, F., Kok, P., 2021. Hippocampal representations switch from errors to predictions during acquisition of predictive associations (preprint). Neuroscience. https://doi.org/10.1101/2021.09.21.461228

Alain, C., Arnott, S.R., Picton, T.W., 2001. Bottom-up and top-down influences on auditory scene analysis: Evidence from eventrelated brain potentials. Journal of Experimental Psychology: Human Perception and Performance 27, 1071-1089.

Alba-Ferrara, L., Hausmann, M., Mitchell, R.L., Weis, S., 2011. The Neural Correlates of Emotional Prosody Comprehension: Disentangling Simple from Complex Emotion. PLoS ONE 6, e28701. https://doi.org/10.1371/journal.pone.0028701

Alberto, G.E., Stapleton-Kotloski, J.R., Klorig, D.C., Rogers, E.R., Constantinidis, C., Daunais, J.B., Godwin, D.W., 2021. MEG source imaging detects optogenetically-induced activity in cortical and subcortical networks. Nat Commun 12, 5259. https://doi.org/10.1038/s41467-021-25481-y

Alluri, V., Brattico, E., Toiviainen, P., Burunat, I., Bogert, B., Numminen, J., Kliuchko, M., 2015. Musical expertise modulates functional connectivity of limbic regions during continuous music listening. Psychomusicology: Music, Mind, and Brain 25, 443-454. https://doi.org/10.1037/pmu0000124

Alluri, V., Toiviainen, P., Burunat, I., Kliuchko, M., Vuust, P., Brattico, E., 2017. Connectivity patterns during music listening: Evidence for action-based processing in musicians: Connectivity Patterns During Music Listening. Human Brain Mapping 38, 2955-2970. https://doi.org/10.1002/hbm.23565

Altafullah, I., Halgren, E., Stapleton, J.M., Crandall, P.H., 1986. Interictal spike-wave complexes in the human medial temporal lobe: Typical topography and comparisons with cognitive potentials. Electroencephalography and Clinical Neurophysiology 63, 503-516.

Alvernhe, A., Save, E., Poucet, B., 2011. Local remapping of place cell firing in the Tolman detour task. European Journal of Neuroscience 33, 1696-1705. https://doi.org/10.1111/j.1460-9568.2011.07653.x

Amaral, D.G., Insausti, R., Cowan, W.M., 1983. Evidence for a direct projection from the superior temporal gyrus to the entorhinal cortex in the monkey. Brain Research 275, 263-277. https://doi.org/10.1016/0006-8993(83)90987-3

Ambrosini, M.V., Mariucci, G., Tantucci, M., Van Hooijdonk, L., Ammassari-Teule, M., 2005. Hippocampal 72-kDa heat shock protein expression varies according to mice learning performance independently from chronic exposure to stress. Hippocampus 15, 413-417. https://doi.org/10.1002/hipo.20069

Anderson, M.I., Jeffery, K.J., 2003. Heterogeneous Modulation of Place Cell Firing by Changes in Context. J. Neurosci. 23, 8827-8835. https://doi.org/10.1523/JNEUROSCI.23-26-08827.2003

Andin, J., Holmer, E., 2022. Reorganization of large-scale brain networks in deaf signing adults: The role of auditory cortex in functional reorganization following deafness. Neuropsychologia 166, 108139. https://doi.org/10.1016/j.neuropsychologia.2021.108139

Angelucci, F., Fiore, M., Ricci, E., Padua, L., Sabino, A., Tonali, P.A., 2007. Investigating the neurobiology of music: brainderived neurotrophic factor modulation in the hippocampus of young adult mice: Behavioural Pharmacology 18, $491-$ 496. https://doi.org/10.1097/FBP.0b013e3282d28f50

Armstrong, N.M., An, Y., Doshi, J., Erus, G., Ferrucci, L., Davatzikos, C., Deal, J.A., Lin, F.R., Resnick, S.M., 2019. Association of Midlife Hearing Impairment With Late-Life Temporal Lobe Volume Loss. JAMA Otolaryngology-Head \& Neck Surgery 145, 794. https://doi.org/10.1001/jamaoto.2019.1610

Arnal, L.H., Kleinschmidt, A., Spinelli, L., Giraud, A.-L., Mégevand, P., 2019. The rough sound of salience enhances aversion through neural synchronisation. Nature Communications 10. https://doi.org/10.1038/s41467-019-11626-7

Aronov, D., Nevers, R., Tank, D.W., 2017. Mapping of a non-spatial dimension by the hippocampal-entorhinal circuit. Nature 543, 719-722. https://doi.org/10.1038/nature21692

Azman, K.F., Zakaria, R., Abdul Aziz, C.B., Othman, Z., 2016. Tualang Honey Attenuates Noise Stress-Induced Memory Deficits in Aged Rats. Oxidative Medicine and Cellular Longevity 2016, 1-11. https://doi.org/10.1155/2016/1549158

Babiloni, C., Vecchio, F., Mirabella, G., Buttiglione, M., Sebastiano, F., Picardi, A., Di Gennaro, G., Quarato, P.P., Grammaldo, L.G., Buffo, P., Esposito, V., Manfredi, M., Cantore, G., Eusebi, F., 2009. Hippocampal, amygdala, and neocortical synchronization of theta rhythms is related to an immediate recall during rey auditory verbal learning test. Human 
Brain Mapping 30, 2077-2089. https://doi.org/10.1002/hbm.20648

Baird, A., Samson, S., 2009. Memory for Music in Alzheimer's Disease: Unforgettable? Neuropsychology Review 19, 85-101. https://doi.org/10.1007/s11065-009-9085-2

Baker, C.M., Burks, J.D., Briggs, R.G., Milton, C.K., Conner, A.K., Glenn, C.A., Sali, G., McCoy, T.M., Battiste, J.D., O’Donoghue, D.L., Sughrue, M.E., 2018. A Connectomic Atlas of the Human Cerebrum—Chapter 6: The Temporal Lobe. Operative Neurosurgery 15, S245-S294. https://doi.org/10.1093/ons/opy260

Baldassano, C., Chen, J., Zadbood, A., Pillow, J.W., Hasson, U., Norman, K.A., 2017. Discovering Event Structure in Continuous Narrative Perception and Memory. Neuron 95, 709-721.e5. https://doi.org/10.1016/j.neuron.2017.06.041

Bang, S.J., Brown, T.H., 2009. Perirhinal cortex supports acquired fear of auditory objects. Neurobiology of Learning and Memory 92, 53-62. https://doi.org/10.1016/j.nlm.2009.01.002

Banks, M.I., Krause, B.M., Berger, D.G., Campbell, D.I., Boes, A.D., Bruss, J.E., Kovach, C.K., Kawasaki, H., Steinschneider, M., Nourski, K.V., 2022. Functional geometry of auditory cortical resting state networks derived from intracranial electrophysiology. bioRxiv. https://doi.org/10.1101/2022.02.06.479292

Banquet, J.-P., Gaussier, P., Cuperlier, N., Hok, V., Save, E., Poucet, B., Quoy, M., Wiener, S.I., 2021. Time as the fourth dimension in the hippocampus. Progress in Neurobiology 199, 101920. https://doi.org/10.1016/j.pneurobio.2020.101920

Bao, X., Gjorgieva, E., Shanahan, L.K., Howard, J.D., Kahnt, T., Gottfried, J.A., 2019. Grid-like Neural Representations Support Olfactory Navigation of a Two-Dimensional Odor Space. Neuron 102, 1066-1075.e5. https://doi.org/10.1016/j.neuron.2019.03.034

Barascud, N., Pearce, M.T., Griffiths, T.D., Friston, K.J., Chait, M., 2016. Brain responses in humans reveal ideal observer-like sensitivity to complex acoustic patterns. Proceedings of the National Academy of Sciences 113, E616-E625. https://doi.org/10.1073/pnas.1508523113

Barbeau, E.J., Felician, O., Joubert, S., Sontheimer, A., Ceccaldi, M., Poncet, M., 2005. Preserved visual recognition memory in an amnesic patient with hippocampal lesions. Hippocampus 15, 587-596. https://doi.org/10.1002/hipo.20079

Barense, M.D., Henson, R.N.A., Lee, A.C.H., Graham, K.S., 2009. Medial temporal lobe activity during complex discrimination of faces, objects, and scenes: Effects of viewpoint. Hippocampus NA-NA. https://doi.org/10.1002/hipo.20641

Barron, H.C., Auksztulewicz, R., Friston, K., 2020. Prediction and memory: A predictive coding account. Progress in Neurobiology 192, 101821. https://doi.org/10.1016/j.pneurobio.2020.101821

Barzegar, M., Sajjadi, F.S., Talaei, S.A., Hamidi, G., Salami, M., 2015. Prenatal exposure to noise stress: Anxiety, impaired spatial memory, and deteriorated hippocampal plasticity in postnatal life: PRENATAL SOUND STRESS AND COGNITION. Hippocampus 25, 187-196. https://doi.org/10.1002/hipo.22363

Başar, E., Demir, N., Gönder, A., Ungan, P., 1979a. Combined dynamics of EEG and evoked potentials I. Studies of simultaneously recorded EEG-EPograms in the auditory pathway, reticular formation, and hippocampus of the cat brain during the waking stage. Biological Cybernetics 34, 1-19.

Başar, E., Demiralp, T., 1995. Fast rhythms in the hippocampus are a part of the diffuse gamma-response system. Hippocampus 5, 240-241. https://doi.org/10.1002/hipo.450050311

Başar, E., Durusan, R., Gönder, A., Ungan, P., 1979b. Combined dynamics of EEG and evoked potentials II. Studies of simultaneously recorded EEG-EPograms in the auditory pathway, reticular formation, and hippocampus of the cat brain during sleep. Biological Cybernetics 34, 21-30.

Başar, E., Özesmi, Ç., 1972. The hippocampal EEG-activity and a systems analytical interpretation of averaged evoked potentials of the brain. Kybernetik 12, 45-54. https://doi.org/10.1007/BF00289236

Başar, E., Ungan, P., 1973. A component analysis and principles derived for the understanding of evoked potentials of the brain: Studies in the hippocampus. Kybernetik 12,133-140. https://doi.org/10.1007/BF00289165

Başar-Eroglu, C., Başar, E., Schmielau, F., 1991. P300 In Freely Moving Cats with Intracranial Electrodes. International Journal of Neuroscience 60, 215-226. https://doi.org/10.3109/00207459109080641

Basile, B.M., Templer, V.L., Gazes, R.P., Hampton, R.R., 2020. Preserved visual memory and relational cognition performance in monkeys with selective hippocampal lesions. Sci. Adv. 6, eaaz0484. https://doi.org/10.1126/sciadv.aaz0484

Baumann, S., Joly, O., Rees, A., Petkov, C.I., Sun, L., Thiele, A., Griffiths, T.D., 2015. The topography of frequency and time representation in primate auditory cortices. eLife 4, e03256. https://doi.org/10.7554/eLife.03256

Baumgartner, T., Lutz, K., Schmidt, C.F., Jäncke, L., 2006. The emotional power of music: How music enhances the feeling of affective pictures. Brain Research 1075, 151-164. https://doi.org/10.1016/j.brainres.2005.12.065

Beaucousin, V., Lacheret, A., Turbelin, M.-R., Morel, M., Mazoyer, B., Tzourio-Mazoyer, N., 2006. FMRI Study of Emotional Speech Comprehension. Cerebral Cortex 17, 339-352. https://doi.org/10.1093/cercor/bhj151

Beckmann, D., Feldmann, M., Shchyglo, O., Manahan-Vaughan, D., 2020. Hippocampal Synaptic Plasticity, Spatial Memory, and Neurotransmitter Receptor Expression Are Profoundly Altered by Gradual Loss of Hearing Ability. Cerebral Cortex. https://doi.org/10.1093/cercor/bhaa061

Behrens, T.E.J., Muller, T.H., Whittington, J.C.R., Mark, S., Baram, A.B., Stachenfeld, K.L., Kurth-Nelson, Z., 2018. What Is a Cognitive Map? Organizing Knowledge for Flexible Behavior. Neuron 100, 490-509. https://doi.org/10.1016/j.neuron.2018.10.002

Bellace, M., Williams, J.M., Mohamed, F.B., Faro, S.H., 2012. An fMRI Study of the Activation of the Hippocampus by Emotional Memory. International Journal of Neuroscience 123, 121-127. https://doi.org/10.3109/00207454.2012.742894

Bellmund, J.L., Deuker, L., Navarro Schröder, T., Doeller, C.F., 2016. Grid-cell representations in mental simulation. eLife 5. https://doi.org/10.7554/eLife.17089

Bendor, D., Wilson, M.A., 2012. Biasing the content of hippocampal replay during sleep. Nature Neuroscience 15, 1439-1444. https://doi.org/10.1038/nn.3203

Bentaleb, L.A., Beauregard, M., Liddle, P., Stip, E., 2002. Cerebral activity associated with auditory verbal hallucinations: a functional magnetic resonance imaging case study. Journal of Psychiatry 27, 6. 
Ben-Yakov, A., Henson, R.N. (Eds.), 2018. The Hippocampal Film Editor: Sensitivity and Specificity to Event Boundaries in Continuous Experience. J. Neurosci. 38, 10057-10068. https://doi.org/10.1523/JNEUROSCI.0524-18.2018

Berger, T., Alger, B., Thompson, R., 1976. Neuronal substrate of classical conditioning in the hippocampus. Science 192, 483485. https://doi.org/10.1126/science.1257783

Berger, T.W., Laham, R.I., Thompson, R.F., 1980. Hippocampal unit-behavior correlations during classical conditioning. Brain Research 193, 229-248. https://doi.org/10.1016/0006-8993(80)90960-9

Berger, T.W., Orr, W.B., 1983. Hippocampectomy selectively disrupts discrimination reversal conditioning of the rabbit nictitating membrane response. Behavioural Brain Research 8, 49-68. https://doi.org/10.1016/0166-4328(83)90171-7

Berger, T.W., Orr, W.B., 1982. Role of the hippocampus in reversal learning of the rabbit nictitating membrane response, in: Woody (Ed.), Conditioning: Representation of Involved Neural Functions. p. 12.

Berger, T.W., Thompson, R.F., 1982. Hippocampal cellular plasticity during extinction of classically conditioned nictitating membrane behavior. Behavioural Brain Research 4, 63-76. https://doi.org/10.1016/0166-4328(82)90165-6

Bergmann, E., Zur, G., Bershadsky, G., Kahn, I., 2016. The Organization of Mouse and Human Cortico-Hippocampal Networks Estimated by Intrinsic Functional Connectivity. Cereb. Cortex 26, 4497-4512. https://doi.org/10.1093/cercor/bhw327

Berry, S.D., Seager, M.A., 2001. Hippocampal Theta Oscillations and Classical Conditioning. Neurobiology of Learning and Memory 76, 298-313. https://doi.org/10.1006/nlme.2001.4025

Beylin, A.V., Gandhi, C.C., Wood, G.E., Talk, A.C., Matzel, L.D., Shors, T.J., 2001. The Role of the Hippocampus in Trace Conditioning: Temporal Discontinuity or Task Difficulty? Neurobiology of Learning and Memory 76, 447-461. https://doi.org/10.1006/nlme.2001.4039

Bhatt, J.M., Bhattacharyya, N., Lin, H.W., 2017. Relationships between tinnitus and the prevalence of anxiety and depression: Tinnitus and Mood Disorders. The Laryngoscope 127, 466-469. https://doi.org/10.1002/lary.26107

Bickford, P.C., Luntz-Leybman, V., Freedman, R., 1993. Auditory sensory gating in the rat hippocampus: modulation by brainstem activity. Brain Research 607, 33-38. https://doi.org/10.1016/0006-8993(93)91486-C

Bickford-Wimer, P.C., Nagamoto, H., Johnson, R., Adler, L.E., Egan, M., Rose, G.M., Freedman, R., 1990. Auditory sensory gating in hippocampal neurons: A model system in the rat. Biological Psychiatry 27, 183-192. https://doi.org/10.1016/0006-3223(90)90648-L

Billig, A.J., Davis, M.H., Carlyon, R.P., 2018. Neural Decoding of Bistable Sounds Reveals an Effect of Intention on Perceptual Organization. The Journal of Neuroscience 38, 2844-2853. https://doi.org/10.1523/JNEUROSCI.3022-17.2018

Bizley, J.K., Cohen, Y.E., 2013. The what, where and how of auditory-object perception. Nature Reviews Neuroscience 14, 693 707. https://doi.org/10.1038/nrn3565

Bizley, J.K., Walker, K.M.M., Silverman, B.W., King, A.J., Schnupp, J.W.H., 2009. Interdependent Encoding of Pitch, Timbre, and Spatial Location in Auditory Cortex. Journal of Neuroscience 29, 2064-2075. https://doi.org/10.1523/JNEUROSCI.4755-08.2009

Blank, H., Spangenberg, M., Davis, M.H., 2018. Neural Prediction Errors Distinguish Perception and Misperception of Speech. J. Neurosci. 38, 6076-6089. https://doi.org/10.1523/JNEUROSCI.3258-17.2018

Blaxton, T.A., Zeffiro, T.A., Gabrieli, J.D.E., Bookheimer, S.Y., Carrillo, M.C., Theodore, W.H., Disterhoft, J.F., 1996. Functional Mapping of Human Learning: A Positron Emission Tomography Activation Study of Eyeblink Conditioning. The Journal of Neuroscience 16, 4032-4040. https://doi.org/10.1523/JNEUROSCI.16-12-04032.1996

Blood, A.J., Zatorre, R.J., 2001. Intensely pleasurable responses to music correlate with activity in brain regions implicated in reward and emotion. Proceedings of the National Academy of Sciences 98, 11818-11823. https://doi.org/10.1073/pnas.191355898

Blood, A.J., Zatorre, R.J., Bermudez, P., Evans, A.C., 1999. Emotional responses to pleasant and unpleasant music correlate with activity in paralimbic brain regions. Nature Neuroscience 2, 382-387. https://doi.org/10.1038/7299

Bonetti, L., Brattico, E., Carlomagno, F., Donati, G., Cabral, J., Haumann, N.T., Deco, G., Vuust, P., Kringelbach, M.L., 2021. Rapid encoding of musical tones discovered in whole-brain connectivity. NeuroImage 245, 118735. https://doi.org/10.1016/j.neuroimage.2021.118735

Bonora, A., Benuzzi, F., Monti, G., Mirandola, L., Pugnaghi, M., Nichelli, P., Meletti, S., 2011. Recognition of emotions from faces and voices in medial temporal lobe epilepsy. Epilepsy \& Behavior 20, 648-654. https://doi.org/10.1016/j.yebeh.2011.01.027

Boon, M.E., Melis, R.J.F., Rikkert, M.O., Kessels, R.P.C., 2011. Atrophy in the Medial Temporal Lobe is Specifically Associated With Encoding and Storage of Verbal Information in MCI and Alzheimer Patients. Journal of Neurology Research. https://doi.org/10.4021/jnr18w

Borders, A.A., Aly, M., Parks, C.M., Yonelinas, A.P., 2017. The hippocampus is particularly important for building associations across stimulus domains. Neuropsychologia 99, 335-342. https://doi.org/10.1016/j.neuropsychologia.2017.03.032

Bordi, F., LeDoux, J.E., 1994. Response properties of single units in areas of rat auditory thalamus that project to the amygdala. Experimental Brain Research 98, 275-286.

Born, J., Wilhelm, I., 2012. System consolidation of memory during sleep. Psychological Research 76, 192-203. https://doi.org/10.1007/s00426-011-0335-6

Boutros, N.N., Mears, R., Pflieger, M.E., Moxon, K.A., Ludowig, E., Rosburg, T., 2008. Sensory gating in the human hippocampal and rhinal regions: Regional differences. Hippocampus 18, 310-316. https://doi.org/10.1002/hipo.20388

Boyen, K., Langers, D.R.M., de Kleine, E., van Dijk, P., 2013. Gray matter in the brain: Differences associated with tinnitus and hearing loss. Hearing Research 295, 67-78. https://doi.org/10.1016/j.heares.2012.02.010

Braak, H., Braak, E., 1991. Neuropathological stageing of Alzheimer-related changes. Acta Neuropathol 82, 239-259. https://doi.org/10.1007/BF00308809

Brady, J.V., Hunt, H.F., 1955. An Experimental Approach to the Analysis of Emotional Behavior. The Journal of Psychology 40, 313-324. https://doi.org/10.1080/00223980.1955.9712986 
Brady, J.V., Schreiner, L., Geller, I., Kling, A., 1954. Subcortical mechanisms in emotional behavior: The effect of rhinencephalic injury upon the acquisition and retention of a conditioned avoidance response in cats. Journal of Comparative and Physiological Psychology.

Brankačk, J., Buzsáki, G., 1986. Hippocampal responses evoked by tooth pulp and acoustic stimulation: Depth profiles and effect of behavior. Brain Research 378, 303-314. https://doi.org/10.1016/0006-8993(86)90933-9

Brankačk, J., Seidenbecher, T., M ller-G rtner, H.-W., 1996. Task-relevant late positive component in rats: Is it related to hippocampal theta rhythm? Hippocampus 6, 475-482. https://doi.org/10.1002/(SICI)1098-1063(1996)6:5<475::AIDHIPO1>3.0.CO;2-I

Brattico, E., Bonetti, L., Ferretti, G., Vuust, P., Matrone, C., 2021. Putting Cells in Motion: Advantages of Endogenous Boosting of BDNF Production. Cells 10, 183. https://doi.org/10.3390/cells10010183

Bregman, A.S., 1990. Auditory Scene Analysis: The Perceptual Organization of Sound. MIT Press, Cambridge.

Britton, K.T., S. Segal, D., Kuczenski, R., Hauger, R., 1992. Dissociation between in vivo hippocampal norepinephrine response and behavioral/neuroendocrine responses to noise stress in rats. Brain Research 574, 125-130. https://doi.org/10.1016/0006-8993(92)90808-M

Brown, K.A., Buchwald, J.S., 1973. Acoustic responses and plasticity of limbic units in cats. Experimental Neurology 40, 608631. https://doi.org/10.1016/0014-4886(73)90099-X

Brown, M.W., Aggleton, J.P., 2001. Recognition memory: What are the roles of the perirhinal cortex and hippocampus? Nature Reviews Neuroscience 2, 51-61. https://doi.org/10.1038/35049064

Brown, S., Martinez, M.J., Parsons, L.M., 2004. Passive music listening spontaneously engages limbic and paralimbic systems: NeuroReport 15, 2033-2037. https://doi.org/10.1097/00001756-200409150-00008

Brun, V.H., Solstad, T., Kjelstrup, K.B., Fyhn, M., Witter, M.P., Moser, E.I., Moser, M.-B., 2008. Progressive increase in grid scale from dorsal to ventral medial entorhinal cortex. Hippocampus 18, 1200-1212. https://doi.org/10.1002/hipo.20504

Buffalo, E.A., 2015. Bridging the gap between spatial and mnemonic views of the hippocampal formation. Hippocampus 25, 713-718. https://doi.org/10.1002/hipo.22444

Burunat, I., Alluri, V., Toiviainen, P., Numminen, J., Brattico, E., 2014. Dynamics of brain activity underlying working memory for music in a naturalistic condition. Cortex 57, 254-269. https://doi.org/10.1016/j.cortex.2014.04.012

Burunat, I., Brattico, E., Hartmann, M., Vuust, P., Särkämö, T., Toiviainen, P., 2018. Musical training predicts cerebellohippocampal coupling during music listening. Psychomusicology: Music, Mind, and Brain 28, 152-163. https://doi.org/10.1037/pmu0000215

Burwell, R.D., Amaral, D.G., 1998. Cortical afferents of the perirhinal, postrhinal, and entorhinal cortices of the rat. The Journal of Comparative Neurology 398, 179-205. https://doi.org/10.1002/(SICI)1096-9861(19980824)398:2<179::AIDCNE3 $>3.0 . \mathrm{CO} ; 2-\mathrm{Y}$

Butler, A.J., James, K.H., 2011. Cross-modal versus within-modal recall: Differences in behavioral and brain responses. Behavioural Brain Research. https://doi.org/10.1016/j.bbr.2011.06.017

Buzsáki, G., 2015. Hippocampal sharp wave-ripple: A cognitive biomarker for episodic memory and planning. Hippocampus 25, 1073-1188. https://doi.org/10.1002/hipo.22488

Buzsáki, G., 2002. Theta Oscillations in the Hippocampus. Neuron 33, 325-340. https://doi.org/10.1016/S0896-6273(02)00586$\mathrm{X}$

Buzsáki, G., Lai-Wo S., L., Vanderwolf, C.H., 1983. Cellular bases of hippocampal EEG in the behaving rat. Brain Research Reviews 6, 139-171. https://doi.org/10.1016/0165-0173(83)90037-1

Campeau, S., Watson, S.J., 1997. Neuroendocrine and Behavioral Responses and Brain Pattern of c-fos Induction Associated with Audiogenic Stress. Journal of Neuroendocrinology 9, 577-588. https://doi.org/10.1046/j.13652826.1997.00593.x

Carpenter-Thompson, J.R., Akrofi, K., Schmidt, S.A., Dolcos, F., Husain, F.T., 2014. Alterations of the emotional processing system may underlie preserved rapid reaction time in tinnitus. Brain Research 1567, $28-41$. https://doi.org/10.1016/j.brainres.2014.04.024

Catenoix, H., Magnin, M., Mauguière, F., Ryvlin, P., 2011. Evoked potential study of hippocampal efferent projections in the human brain. Clinical Neurophysiology 122, 2488-2497. https://doi.org/10.1016/j.clinph.2011.05.007

Cavaco, S., Feinstein, J.S., van Twillert, H., Tranel, D., 2012. Musical memory in a patient with severe anterograde amnesia. Journal of Clinical and Experimental Neuropsychology 34, 1089-1100. https://doi.org/10.1080/13803395.2012.728568

Cavazzuti, V., Winston, K., Baker, R., Welch, K., 1980. Psychological changes following surgery for tumors in the temporal lobe. Journal of Neurosurgery 53, 618-626. https://doi.org/10.3171/jns.1980.53.5.0618

Cave, C.B., Squire, L.R., 1992. Intact verbal and nonverbal short-term memory following damage to the human hippocampus. Hippocampus 2, 151-163. https://doi.org/10.1002/hipo.450020207

Cazard, P., Buser, P., 1963. Modification des réponses sensorielles corticales par stimulation de l'hippocampe dorsal chez le lapin. Electroencephalography and Clinical Neurophysiology 15, 413-425.

Cenquizca, L.A., Swanson, L.W., 2007. Spatial organization of direct hippocampal field CA1 axonal projections to the rest of the cerebral cortex. Brain Research Reviews 56, 1-26. https://doi.org/10.1016/j.brainresrev.2007.05.002

Chaddock-Heyman, L., Loui, P., Weng, T.B., Weisshappel, R., McAuley, E., Kramer, A.F., 2021. Musical Training and Brain Volume in Older Adults. Brain Sciences 11, 50. https://doi.org/10.3390/brainsci11010050

Chadwick, M.J., Mullally, S.L., Maguire, E.A., 2013. The hippocampus extrapolates beyond the view in scenes: An fMRI study of boundary extension. Cortex 49, 2067-2079. https://doi.org/10.1016/j.cortex.2012.11.010

Chan, D., Suk, H.-J., Jackson, B., Milman, N.P., Stark, D., Klerman, E.B., Kitchener, E., Fernandez Avalos, V.S., Banerjee, A., Beach, S.D., Blanchard, J., Stearns, C., Boes, A., Uitermarket, B., Gander, P., Howard, M., Sternberg, E.J., NietoCastanon, A., Anteraper, S., Whitfield-Gabrieli, S., Brown, E.N., Boyden, E.S., Dickerson, B., Tsai, L.-H., 2021. Gamma Frequency Sensory Stimulation in Probable Mild Alzheimer's Dementia Patients: Results of a Preliminary 
Clinical Trial. bioRxiv 34 .

Chan, R.W., Leong, A.T.L., Ho, L.C., Gao, P.P., Wong, E.C., Dong, C.M., Wang, X., He, J., Chan, Y.-S., Lim, L.W., Wu, E.X., 2017. Low-frequency hippocampal-cortical activity drives brain-wide resting-state functional MRI connectivity. Proceedings of the National Academy of Sciences 114, E6972-E6981. https://doi.org/10.1073/pnas.1703309114

Chao, L.L., Knight, R.T., 1995. Human prefrontal lesions increase distractibility to irrelevant sensory inputs. NeuroReport 6, $1605-1610$.

Chapin, H., Jantzen, K., Scott Kelso, J.A., Steinberg, F., Large, E., 2010a. Dynamic Emotional and Neural Responses to Music Depend on Performance Expression and Listener Experience. PLoS ONE 5, e13812. https://doi.org/10.1371/journal.pone.0013812

Chapin, H., Zanto, T., Jantzen, K.J., Kelso, S.J.A., Steinberg, F., Large, E.W., 2010b. Neural Responses to Complex Auditory Rhythms: The Role of Attending. Frontiers in Psychology 1. https://doi.org/10.3389/fpsyg.2010.00224

Chatani, H., Hagiwara, K., Hironaga, N., Ogata, K., Shigeto, H., Morioka, T., Sakata, A., Hashiguchi, K., Murakami, N., Uehara, T., Kira, J., Tobimatsu, S., 2016. Neuromagnetic evidence for hippocampal modulation of auditory processing. NeuroImage 124, 256-266. https://doi.org/10.1016/j.neuroimage.2015.09.006

Chaudhury, S., Jain, S., Wadhwa, S., 2010. Expression of Synaptic Proteins in the Hippocampus and Spatial Learning in Chicks following Prenatal Auditory Stimulation. Developmental Neuroscience 32, 114-124. https://doi.org/10.1159/000279758

Chaudhury, S., Nag, T.C., Wadhwa, S., 2008. Calbindin D-28K and parvalbumin expression in embryonic chick hippocampus is enhanced by prenatal auditory stimulation. Brain Research 1191, 96-106. https://doi.org/10.1016/j.brainres.2007.11.021

Chaudhury, S., Nag, T.C., Wadhwa, S., 2006. Prenatal acoustic stimulation influences neuronal size and the expression of calcium-binding proteins (calbindin D-28K and parvalbumin) in chick hippocampus. Journal of Chemical Neuroanatomy 32, 117-126. https://doi.org/10.1016/j.jchemneu.2006.07.002

Chaudhury, S., Wadhwa, S., 2009. Prenatal auditory stimulation alters the levels of CREB mRNA, p-CREB and BDNF expression in chick hippocampus. International Journal of Developmental Neuroscience 27, 583-590. https://doi.org/10.1016/j.ijdevneu.2009.06.004

Chen, G.-D., Radziwon, K.E., Kashanian, N., Manohar, S., Salvi, R., 2014. Salicylate-Induced Auditory Perceptual Disorders and Plastic Changes in Nonclassical Auditory Centers in Rats. Neural Plasticity 2014, 1-18. https://doi.org/10.1155/2014/658741

Chen, Y., Rex, C.S., Rice, C.J., Dube, C.M., Gall, C.M., Lynch, G., Baram, T.Z., 2010. Correlated memory defects and hippocampal dendritic spine loss after acute stress involve corticotropin-releasing hormone signaling. Proceedings of the National Academy of Sciences 107, 13123-13128. https://doi.org/10.1073/pnas.1003825107

Chen, Y.-C., Li, X., Liu, L., Wang, J., Lu, C.-Q., Yang, M., Jiao, Y., Zang, F.-C., Radziwon, K., Chen, G.-D., Sun, W., Krishnan Muthaiah, V.P., Salvi, R., Teng, G.-J., 2015. Tinnitus and hyperacusis involve hyperactivity and enhanced connectivity in auditory-limbic-arousal-cerebellar network. eLife 4. https://doi.org/10.7554/eLife.06576

Chen, Y.-C., Wang, F., Wang, J., Bo, F., Xia, W., Gu, J.-P., Yin, X., 2017a. Resting-State Brain Abnormalities in Chronic Subjective Tinnitus: A Meta-Analysis. Frontiers in Human Neuroscience 11. https://doi.org/10.3389/fnhum.2017.00022

Chen, Y.-C., Xia, W., Chen, H., Feng, Y., Xu, J.-J., Gu, J.-P., Salvi, R., Yin, X., 2017b. Tinnitus distress is linked to enhanced resting-state functional connectivity from the limbic system to the auditory cortex: Altered Effective Connectivity in Tinnitus. Human Brain Mapping 38, 2384-2397. https://doi.org/10.1002/hbm.23525

Chen, Y.-C., Yong, W., Xing, C., Feng, Y., Haidari, N.A., Xu, J.-J., Gu, J.-P., Yin, X., Wu, Y., 2020. Directed functional connectivity of the hippocampus in patients with presbycusis. Brain Imaging and Behavior 14, 917-926. https://doi.org/10.1007/s11682-019-00162-z

Cheng, D.T., Disterhoft, J.F., Power, J.M., Ellis, D.A., Desmond, J.E., 2008. Neural substrates underlying human delay and trace eyeblink conditioning. Proceedings of the National Academy of Sciences 105, 8108-8113. https://doi.org/10.1073/pnas.0800374105

Cheng, L., Wang, S.-H., Chen, Q.-C., Liao, X.-M., 2011. Moderate noise induced cognition impairment of mice and its underlying mechanisms. Physiology \& Behavior 104, 981-988. https://doi.org/10.1016/j.physbeh.2011.06.018

Cheng, L., Wang, S.-H., Huang, Y., Liao, X.-M., 2016. The hippocampus may be more susceptible to environmental noise than the auditory cortex. Hearing Research 333, 93-97. https://doi.org/10.1016/j.heares.2016.01.001

Cheung, V.K.M., Harrison, P.M.C., Meyer, L., Pearce, M.T., Haynes, J.-D., Koelsch, S., 2019. Uncertainty and Surprise Jointly Predict Musical Pleasure and Amygdala, Hippocampus, and Auditory Cortex Activity. Current Biology 29. https://doi.org/10.1016/j.cub.2019.09.067

Chikahisa, S., Sei, H., Morishima, M., Sano, A., Kitaoka, K., Nakaya, Y., Morita, Y., 2006. Exposure to music in the perinatal period enhances learning performance and alters BDNF/TrkB signaling in mice as adults. Behavioural Brain Research 169, 312-319. https://doi.org/10.1016/j.bbr.2006.01.021

Christison-Lagay, K.L., Gifford, A.M., Cohen, Y.E., 2015. Neural correlates of auditory scene analysis and perception. International Journal of Psychophysiology 95, 238-245. https://doi.org/10.1016/j.ijpsycho.2014.03.004

Clark, I.A., Monk, A.M., Hotchin, V., Pizzamiglio, G., Liefgreen, A., Callaghan, M.F., Maguire, E.A., 2020. Does hippocampal volume explain performance differences on hippocampal-dependant tasks? NeuroImage 221, 117211. https://doi.org/10.1016/j.neuroimage.2020.117211

Clark, R.E., Squire, 1998. Classical Conditioning and Brain Systems: The Role of Awareness. Science 280, 77-81. https://doi.org/10.1126/science.280.5360.77

Clark, R.E., Squire, L.R., 2013. Similarity in form and function of the hippocampus in rodents, monkeys, and humans. Proceedings of the National Academy of Sciences 110, 10365-10370. https://doi.org/10.1073/pnas.1301225110

Clos, M., Langner, R., Meyer, M., Oechslin, M.S., Zilles, K., Eickhoff, S.B., 2014. Effects of prior information on decoding degraded speech: An fMRI study. Hum. Brain Mapp 35, 61-74. https://doi.org/10.1002/hbm.22151 
Cohen, N.J., Eichenbaum, H., 1993. Memory, Amnesia, and the Hippocampal System. MIT Press, Cambridge.

Cole, S., Stone, A.D., Petrovich, G.D., 2017. The dorsomedial striatum mediates Pavlovian appetitive conditioning and food consumption. Behavioral Neuroscience 131, 447-453. https://doi.org/10.1037/bne0000216

Colgin, L.L., Denninger, T., Fyhn, M., Hafting, T., Bonnevie, T., Jensen, O., Moser, M.-B., Moser, E.I., 2009. Frequency of gamma oscillations routes flow of information in the hippocampus. Nature 462, 353-357. https://doi.org/10.1038/nature08573

Constantinescu, A.O., OReilly, J.X., Behrens, T.E.J., 2016. Organizing conceptual knowledge in humans with a gridlike code. Science 352, 1464-1468. https://doi.org/10.1126/science.aaf0941

Cooper, R.A., Ritchey, M., 2020. Progression from Feature-Specific Brain Activity to Hippocampal Binding during Episodic Encoding. The Journal of Neuroscience 40, 1701-1709. https://doi.org/10.1523/JNEUROSCI.1971-19.2019

Cooper, R.A., Ritchey, M., 2019. Cortico-hippocampal network connections support the multidimensional quality of episodic memory. eLife 8. https://doi.org/10.7554/eLife.45591

Copolov, D.L., Seal, M.L., Maruff, P., Ulusoy, R., Wong, M.T.H., Tochon-Danguy, H.J., Egan, G.F., 2003. Cortical activation associated with the experience of auditory hallucinations and perception of human speech in schizophrenia: a PET correlation study. Psychiatry Research: Neuroimaging 122, 139-152. https://doi.org/10.1016/S0925-4927(02)00121-X

Coras, R., Pauli, E., Li, J., Schwarz, M., Rössler, K., Buchfelder, M., Hamer, H., Stefan, H., Blumcke, I., 2014. Differential influence of hippocampal subfields to memory formation: insights from patients with temporal lobe epilepsy. Brain 137, 1945-1957. https://doi.org/10.1093/brain/awu100

Corcoran, K.A., Maren, S., 2001. Hippocampal Inactivation Disrupts Contextual Retrieval of Fear Memory after Extinction. The Journal of Neuroscience 21, 1720-1726. https://doi.org/10.1523/JNEUROSCI.21-05-01720.2001

Corkin, S., Amaral, D.G., González, R.G., Johnson, K.A., Hyman, B.T., 1997. H. M.’s Medial Temporal Lobe Lesion: Findings from Magnetic Resonance Imaging. The Journal of Neuroscience 17, 3964-3979. https://doi.org/10.1523/JNEUROSCI.17-10-03964.1997

Corrigall, K.A., Schellenberg, E.G., Misura, N.M., 2013. Music Training, Cognition, and Personality. Frontiers in Psychology 4. https://doi.org/10.3389/fpsyg.2013.00222

Cousins, J.N., El-Deredy, W., Parkes, L.M., Hennies, N., Lewis, P.A., 2016. Cued Reactivation of Motor Learning during Sleep Leads to Overnight Changes in Functional Brain Activity and Connectivity. PLoS Biol 14, e1002451. https://doi.org/10.1371/journal.pbio.1002451

Covington, N.V., Brown-Schmidt, S., Duff, M.C., 2018. The Necessity of the Hippocampus for Statistical Learning. Journal of Cognitive Neuroscience 30, 680-697. https://doi.org/10.1162/jocn_a_01228

Cromwell, H.C., Mears, R.P., Wan, L., Boutros, N.N., 2008. Sensory Gating: A Translational Effort from Basic to Clinical Science. Clinical EEG and Neuroscience 39, 69-72. https://doi.org/10.1177/155005940803900209

Crönlein, T., Langguth, B., Geisler, P., Hajak, G., 2007. Tinnitus and insomnia, in: Progress in Brain Research. Elsevier, pp. $227-$ 233. https://doi.org/10.1016/S0079-6123(07)66021-X

Crottaz-Herbette, S., Lau, K.M., Glover, G.H., Menon, V., 2005. Hippocampal involvement in detection of deviant auditory and visual stimuli. Hippocampus 15, 132-139. https://doi.org/10.1002/hipo.20039

Crowley, R., Bendor, D., Javadi, A.-H., 2019. A review of neurobiological factors underlying the selective enhancement of memory at encoding, consolidation, and retrieval. Progress in Neurobiology 179, 101615. https://doi.org/10.1016/j.pneurobio.2019.04.004

Csépe, V., Karmos, G., Molnár, M., 1989. Subcortical Evoked Potential Correlates of Early Information Processing: Mismatch Negativity in Cats, in: Brain Dynamics: Progress and Perspectives. Springer, Berlin.

Cuddy, L.L., Sikka, R., Vanstone, A., 2015. Preservation of musical memory and engagement in healthy aging and Alzheimer's disease: Musical memory in Alzheimer's disease. Annals of the New York Academy of Sciences 1337, $223-231$. https://doi.org/10.1111/nyas.12617

Cui, B., Li, K., Gai, Z., She, X., Zhang, N., Xu, C., Chen, X., An, G., Ma, Q., Wang, R., 2015. Chronic Noise Exposure Acts Cumulatively to Exacerbate Alzheimer's Disease-Like Amyloid- $\beta$ Pathology and Neuroinflammation in the Rat Hippocampus. Scientific Reports 5. https://doi.org/10.1038/srep12943

Cui, B., Wu, M., She, X., 2009. Effects of Chronic Noise Exposure on Spatial Learning and Memory of Rats in Relation to Neurotransmitters and NMDAR2B Alteration in the Hippocampus. Journal of Occupational Health 51, 152-158. https://doi.org/10.1539/joh.L8084

Cui, B., Wu, M., She, X., Liu, H., 2012a. Impulse noise exposure in rats causes cognitive deficits and changes in hippocampal neurotransmitter signaling and tau phosphorylation. Brain Research 1427, 35-43. https://doi.org/10.1016/j.brainres.2011.08.035

Cui, B., Wu, M.Q., Zhu, L.X., She, X.J., Qiang, M.A., Liu, H.T., 2013. Effect of chronic noise exposure on expression of NMethyl-D-Aspartic acid receptor $2 \mathrm{~B}$ and tau phosphorylation in hippocampus of rats. Biomedical Environmental Science 26, 163-168.

Cui, B., Zhu, L., She, X., Wu, M., Ma, Q., Wang, T., Zhang, N., Xu, C., Chen, X., An, G., Liu, H., 2012b. Chronic noise exposure causes persistence of tau hyperphosphorylation and formation of NFT tau in the rat hippocampus and prefrontal cortex. Experimental Neurology 238, 122-129. https://doi.org/10.1016/j.expneurol.2012.08.028

Cunha, A.O.S., Ceballos, C.C., de Deus, J.L., Leão, R.M., 2018. Long-term high-intensity sound stimulation inhibits h current ( $I_{\mathrm{h}}$ ) in CA1 pyramidal neurons. European Journal of Neuroscience 47, 1401-1413. https://doi.org/10.1111/ejn.13954

Cunha, A.O.S., de Oliveira, J.A.C., Almeida, S.S., Garcia-Cairasco, N., Leão, R.M., 2015. Inhibition of long-term potentiation in the schaffer-CA1 pathway by repetitive high-intensity sound stimulation. Neuroscience 310, 114-127. https://doi.org/10.1016/j.neuroscience.2015.09.040

Curtu, R., Wang, X., Brunton, B.W., Nourski, K.V., 2019. Neural Signatures of Auditory Perceptual Bistability Revealed by Large-Scale Human Intracranial Recordings. J. Neurosci. 39, 6482-6497. https://doi.org/10.1523/JNEUROSCI.065518.2019

Dalton, M.A., Zeidman, P., McCormick, C., Maguire, E.A., 2018. Differentiable Processing of Objects, Associations, and Scenes 
within the Hippocampus. The Journal of Neuroscience 38, 8146-8159. https://doi.org/10.1523/JNEUROSCI.026318.2018

Danker, J.F., Tompary, A., Davachi, L., 2016. Trial-by-Trial Hippocampal Encoding Activation Predicts the Fidelity of Cortical Reinstatement During Subsequent Retrieval. Cerebral Cortex bhw146. https://doi.org/10.1093/cercor/bhw146

Daum, I., Schugens, M.M., Ackermann, H., Lutzenberger, W., Dichgans, J., Birbaumer, N., 1993. Classical conditioning after cerebellar lesions in humans. Behav Neurosci 107, 748-756. https://doi.org/10.1037//0735-7044.107.5.748

Davis, M.H., Di Betta, A.M., Macdonald, M.J.E., Gaskell, M.G., 2009. Learning and Consolidation of Novel Spoken Words. Journal of Cognitive Neuroscience 21, 803-820. https://doi.org/10.1162/jocn.2009.21059

Davis, M.H., Ford, M.A., Kherif, F., Johnsrude, I.S., 2011. Does Semantic Context Benefit Speech Understanding through "Top-Down" Processes? Evidence from Time-resolved Sparse fMRI. Journal of Cognitive Neuroscience 23, 3914 3932. https://doi.org/10.1162/jocn_a_00084

Davis, M.H., Johnsrude, I.S., 2003. Hierarchical Processing in Spoken Language Comprehension. The Journal of Neuroscience 23, 3423-3431. https://doi.org/10.1523/JNEUROSCI.23-08-03423.2003

Dayan, P., 1993. Improving Generalization for Temporal Difference Learning: The Successor Representation. Neural Computation 5, 613-624. https://doi.org/10.1162/neco.1993.5.4.613

de Deus, J.L., Amorim, M.R., Ribeiro, A.B., Barcellos-Filho, P.C.G., Ceballos, C.C., Branco, L.G.S., Cunha, A.O.S., Leão, R.M., 2021. Loss of Brain-Derived Neurotrophic Factor Mediates Inhibition of Hippocampal Long-Term Potentiation by High-Intensity Sound. Cell Mol Neurobiol 41, 751-763. https://doi.org/10.1007/s10571-020-00881-8

de Deus, J.L., Cunha, A.O.S., Terzian, A.L., Resstel, L.B., Elias, L.L.K., Antunes-Rodrigues, J., Almeida, S.S., Leão, R.M., 2017. A single episode of high intensity sound inhibits long-term potentiation in the hippocampus of rats. Scientific Reports 7. https://doi.org/10.1038/s41598-017-14624-1

De Ridder, D., Fransen, H., Francois, O., Sunaert, S., Kovacs, S., Van De Heyning, P., 2006. Amygdalohippocampal involvement in tinnitus and auditory memory. Acta Oto-Laryngologica 126, 50-53. https://doi.org/10.1080/03655230600895580

De Ridder, D., Vanneste, S., Weisz, N., Londero, A., Schlee, W., Elgoyhen, A.B., Langguth, B., 2014. An integrative model of auditory phantom perception: Tinnitus as a unified percept of interacting separable subnetworks. Neuroscience \& Biobehavioral Reviews 44, 16-32. https://doi.org/10.1016/j.neubiorev.2013.03.021

Deadwyler, S.A., West, M.O., Christian, E.P., Hampson, R.E., Foster, T.C., 1985. Sequence-related changes in sensory-evoked potentials in the dentate gyrus: A mechanism for item-specific short-term information storage in the hippocampus. Behavioral and Neural Biology 44, 201-212. https://doi.org/10.1016/S0163-1047(85)90198-0

DeLisi, L.E., S. Buchsbaum, M., Holcomb, H.H., Langston, K.C., King, A.C., Kessler, R., Pickar, D., Carpenter, W.T., Morihisa, J.M., Margolin, R., Weinberger, D.R., 1989. Increased temporal lobe glucose use in chronic schizophrenic patients. Biological Psychiatry 25, 835-851. https://doi.org/10.1016/0006-3223(89)90263-1

Dellacherie, D., Bigand, E., Molin, P., Baulac, M., Samson, S., 2011. Multidimensional scaling of emotional responses to music in patients with temporal lobe resection. Cortex 47,1107-1115. https://doi.org/10.1016/j.cortex.2011.05.007

Dellacherie, D., Ehrlé, N., Samson, S., 2008. Is the neutral condition relevant to study musical emotion in patients? Music Perception: An Interdisciplinary Journal 25, 285-294. https://doi.org/10.1525/mp.2008.25.4.285

Demiralp, T., Başar-Eroglu, C., Başar, E., 1996. Distributed Gamma Band Responses in the Brain Studied in Cortex, Reticular Formation, Hippocampus and Cerebellum. International Journal of Neuroscience 84, 1-13. https://doi.org/10.3109/00207459608987246

Derner, M., Chaieb, L., Dehnen, G., Reber, T.P., Borger, V., Surges, R., Staresina, B.P., Mormann, F., Fell, J., 2021. Auditory Beat Stimulation Modulates Memory-Related Single-Neuron Activity in the Human Medial Temporal Lobe. Brain Sciences 11, 364. https://doi.org/10.3390/brainsci11030364

Derner, M., Dehnen, G., Chaieb, L., Reber, T.P., Borger, V., Surges, R., Staresina, B.P., Mormann, F., Fell, J., 2020. Patterns of single-neuron activity during associative recognition memory in the human medial temporal lobe. NeuroImage 221, 117214. https://doi.org/10.1016/j.neuroimage.2020.117214

Derner, M., Jahanbekam, A., Bauckhage, C., Axmacher, N., Fell, J., 2018. Prediction of memory formation based on absolute electroencephalographic phases in rhinal cortex and hippocampus outperforms prediction based on stimulus-related phase shifts. European Journal of Neuroscience 47, 824-831. https://doi.org/10.1111/ejn.13878

Devlin, J.T., Price, C.J., 2007. Perirhinal Contributions to Human Visual Perception. Current Biology 17, 1484-1488. https://doi.org/10.1016/j.cub.2007.07.066

Dheerendra, P., Baumann, S., Joly, O., Balezeau, F., Petkov, C.I., Thiele, A., Griffiths, T.D., 2021. The Representation of Time Windows in Primate Auditory Cortex. Cerebral Cortex bhab434. https://doi.org/10.1093/cercor/bhab434

Di, G.-Q., Qin, Z.-Q., 2018. Influences of combined traffic noise on the ability of learning and memory in mice. Noise Health 20, 9-15.

Diba, K., Buzsáki, G., 2007. Forward and reverse hippocampal place-cell sequences during ripples. Nature Neuroscience 10, 1241-1242. https://doi.org/10.1038/nn1961

Diederen, K.M.J., Neggers, S.F.W., Daalman, K., Blom, J.D., Goekoop, R., Kahn, R.S., Sommer, I.E.C., 2010. Deactivation of the Parahippocampal Gyrus Preceding Auditory Hallucinations in Schizophrenia. American Journal of Psychiatry 167, 427-435. https://doi.org/10.1176/appi.ajp.2009.09040456

Dierks, T., Linden, D.E.J., Jandl, M., Formisano, E., Goebel, R., Lanfermann, H., Singer, W., 1999. Activation of Heschl's Gyrus during Auditory Hallucinations. Neuron 22, 615-621. https://doi.org/10.1016/S0896-6273(00)80715-1

Dietz, B., Manahan-Vaughan, D., 2017. Hippocampal long-term depression is facilitated by the acquisition and updating of memory of spatial auditory content and requires mGlu5 activation. Neuropharmacology 115, 30-41. https://doi.org/10.1016/j.neuropharm.2016.02.026

Disterhoft, J.F., Segal, M., 1978. Neuron activity in rat hippocampus and motor cortex during discrimination reversal. Brain Research Bulletin 3, 583-588. https://doi.org/10.1016/0361-9230(78)90003-5

Doeller, C.F., Barry, C., Burgess, N., 2010. Evidence for grid cells in a human memory network. Nature 463, 657-661. https://doi.org/10.1038/nature08704 
Dolan, R.J., Fletcher, P.C., 1997. Dissociating prefrontal and hippocampal function in episodic memory encoding. Nature 388, 582-585. https://doi.org/10.1038/41561

Dong, Y., Guo, C.-R., Chen, D., Chen, S.-M., Peng, Y., Song, H., Shi, J.-R., 2018. Association between age-related hearing loss and cognitive decline in C57BL/6J mice. Molecular Medicine Reports 18, 1726-1732. https://doi.org/10.3892/mmr.2018.9118

Donzis, E.J., Rennaker, R.L., Thompson, L.T., 2013. Fear conditioning alters neuron-specific hippocampal place field stability via the basolateral amygdala. Brain Research 1525, 16-25. https://doi.org/10.1016/j.brainres.2013.06.015

Dragoi, G., Tonegawa, S., 2013. Distinct preplay of multiple novel spatial experiences in the rat. Proceedings of the National Academy of Sciences 110, 9100-9105. https://doi.org/10.1073/pnas.1306031110

Dulay, M.F., Schefft, B.K., Fargo, J.D., Privitera, M.D., Yeh, H., 2004. Severity of depressive symptoms, hippocampal sclerosis, auditory memory, and side of seizure focus in temporal lobe epilepsy. Epilepsy \& Behavior 5, 522-531. https://doi.org/10.1016/j.yebeh.2004.04.007

Eggermont, J.J., 2013. Hearing loss, hyperacusis, or tinnitus: What is modeled in animal research? Hearing Research 295, 140149. https://doi.org/10.1016/j.heares.2012.01.005

Ehlers, C.L., Kaneko, W.M., Robledo, P., Lopez, A.L., 1994. Long-latency event-related potentials in rats: Effects of task and stimulus parameters. Neuroscience 62, 759-769. https://doi.org/10.1016/0306-4522(94)90474-X

Ehrlé, N., 2001. Processing of rapid auditory information in epileptic patients with left temporal lobe damage. Neuropsychologia 39, 525-531. https://doi.org/10.1016/S0028-3932(00)00121-4

Eichenbaum, H., 2017. Elements of Information Processing in Hippocampal Neuronal Activity: Space, Time, and Memory, in: Hannula, D.E., Duff, M.C. (Eds.), The Hippocampus from Cells to Systems. Springer International Publishing, Cham, pp. 69-94. https://doi.org/10.1007/978-3-319-50406-3_3

Eidelberg, E., White, J.C., Brazier, M.A.B., 1959. The hippocampal arousal pattern in rabbits. Experimental Neurology 1, 483490. https://doi.org/10.1016/0014-4886(59)90045-7

Eldar, E., Ganor, O., Admon, R., Bleich, A., Hendler, T., 2007. Feeling the Real World: Limbic Response to Music Depends on Related Content. Cerebral Cortex 17, 2828-2840. https://doi.org/10.1093/cercor/bhm011

Enatsu, R., Gonzalez-Martinez, J., Bulacio, J., Kubota, Y., Mosher, J., Burgess, R.C., Najm, I., Nair, D.R., 2015. Connections of the limbic network: A corticocortical evoked potentials study. Cortex 62, 20-33. https://doi.org/10.1016/j.cortex.2014.06.018

Engel, A., Keller, P.E., 2011. The Perception of Musical Spontaneity in Improvised and Imitated Jazz Performances. Frontiers in Psychology 2. https://doi.org/10.3389/fpsyg.2011.00083

Esfahani-Bayerl, N., Finke, C., Kopp, U., Moon, D.-U., Ploner, C.J., 2019. Musical memory and hippocampus revisited: Evidence from a musical layperson with highly selective hippocampal damage. Cortex 119, 519-527. https://doi.org/10.1016/j.cortex.2018.12.023

Ezzyat, Y., Davachi, L., 2014. Similarity Breeds Proximity: Pattern Similarity within and across Contexts Is Related to Later Mnemonic Judgments of Temporal Proximity. Neuron 81, 1179-1189. https://doi.org/10.1016/j.neuron.2014.01.042

Feldman, S., Dafny, N., 1968. Acoustic responses in the hypothalamus. Electroencephalography and Clinical Neurophysiology $25,150-159$.

Felleman, D.J., Van Essen, D.C., 1991. Distributed Hierarchical Processing in the Primate Cerebral Cortex. Cerebral Cortex 1,147. https://doi.org/10.1093/cercor/1.1.1

Fernandes, C., File, S.E., 1993. Beware the builders: Construction noise changes [14C]GABA release and uptake from amygdaloid and hippocampal slices in the rat. Neuropharmacology 32, 1333-1336. https://doi.org/10.1016/00283908(93)90028-2

Ferrarese, C., Mennini, T., Pecora, N., Gobbi, M., Appollonio, I., Bernasconi, P., Frigo, M., Regondi, C., Pierpaoli, C., Frattola, L., Garattini, S., 1991. Acute noise stress in rats increases the levels of diazepam binding inhibitor (DBI) in hippocampus and adrenal gland. Psychopharmacology 103, 339-342. https://doi.org/10.1007/BF02244287

Ferri, S., Meini, C., Guiot, G., Tagliafico, D., Gilli, G., Di Dio, C., 2014. The Effect of Simple Melodic Lines on Aesthetic Experience: Brain Response to Structural Manipulations. Advances in Neuroscience 2014, 1-9. https://doi.org/10.1155/2014/482126

Field, S.E., Rickard, N.S., Toukhsati, S.R., Gibbs, M.E., 2007. Maternal hen calls modulate memory formation in the day-old chick: The role of noradrenaline. Neurobiology of Learning and Memory 88, 321-330. https://doi.org/10.1016/j.nlm.2007.04.001

Finke, C., Esfahani, N.E., Ploner, C.J., 2012. Preservation of musical memory in an amnesic professional cellist. Current Biology 22, R591-R592. https://doi.org/10.1016/j.cub.2012.05.041

Fitzhugh, M.C., Pa, J., 2022. Longitudinal Changes in Resting-State Functional Connectivity and Gray Matter Volume Are Associated with Conversion to Hearing Impairment in Older Adults. JAD 1-14. https://doi.org/10.3233/JAD215288

Fletcher, P.D., Downey, L.E., Witoonpanich, P., Warren, J.D., 2013. The brain basis of musicophilia: evidence from frontotemporal lobar degeneration. Frontiers in Psychology 4. https://doi.org/10.3389/fpsyg.2013.00347

Flores-Gutiérrez, E.O., Díaz, J.-L., Barrios, F.A., Favila-Humara, R., Guevara, M.Á., del Río-Portilla, Y., Corsi-Cabrera, M., 2007. Metabolic and electric brain patterns during pleasant and unpleasant emotions induced by music masterpieces. International Journal of Psychophysiology 65, 69-84. https://doi.org/10.1016/j.ijpsycho.2007.03.004

Fonken, Y.M., Kam, J.W.Y., Knight, R.T., 2019. A differential role for human hippocampus in novelty and contextual processing: Implications for P300. Psychophysiology e13400. https://doi.org/10.1111/psyp.13400

Ford, J.H., Addis, D.R., Giovanello, K.S., 2011. Differential neural activity during search of specific and general autobiographical memories elicited by musical cues. Neuropsychologia 49, 2514-2526. https://doi.org/10.1016/j.neuropsychologia.2011.04.032

Foster, D.J., Wilson, M.A., 2006. Reverse replay of behavioural sequences in hippocampal place cells during the awake state. Nature 440, 680-683. https://doi.org/10.1038/nature04587 
Fowler, H.L., Baker, G.A., Tipples, J., Hare, D.J., Keller, S., Chadwick, D.W., Young, A.W., 2006. Recognition of emotion with temporal lobe epilepsy and asymmetrical amygdala damage. Epilepsy \& Behavior 9, 164-172. https://doi.org/10.1016/j.yebeh.2006.04.013

Fox, S.S., Liebeskind, J.C., O’Brien, J.H., Dingle, R.D.H., 1967. Mechanisms for Limbic Modification of Cerebellar and Cortical Afferent Information, in: Progress in Brain Research. Elsevier, pp. 254-280. https://doi.org/10.1016/S00796123(08)63104-0

Freeman, F., Kramarcy, N., Lee, J., 1973. Discrimination learning and stimulus generalization in rats with hippocampal lesions. Physiology \& Behavior 11, 273-275. https://doi.org/10.1016/0031-9384(73)90362-4

Frenzilli, G., Ryskalin, L., Ferrucci, M., Cantafora, E., Chelazzi, S., Giorgi, F.S., Lenzi, P., Scarcelli, V., Frati, A., Biagioni, F., Gambardella, S., Falleni, A., Fornai, F., 2017. Loud Noise Exposure Produces DNA, Neurotransmitter and Morphological Damage within Specific Brain Areas. Frontiers in Neuroanatomy 11. https://doi.org/10.3389/fnana.2017.00049

Frisk, V., Milner, B., 1990. The role of the left hippocampal region in the acquisition and retention of story content. Neuropsychologia 28, 349-359. https://doi.org/10.1016/0028-3932(90)90061-R

Friston, K., Buzsáki, G., 2016. The Functional Anatomy of Time: What and When in the Brain. Trends in Cognitive Sciences 20, 500-511. https://doi.org/10.1016/j.tics.2016.05.001

Friston, K.J., Liddle, P.F., Frith, C.D., Hirsch, S.R., Frackowiak, R.S.J., 1992. The left medial temporal region and schizophrenia: A PET study. Brain 115, 367-382. https://doi.org/10.1093/brain/115.2.367

Fritz, J., Mishkin, M., Saunders, R.C., 2005. In search of an auditory engram. Proceedings of the National Academy of Sciences 102, 9359-9364. https://doi.org/10.1073/pnas.0503998102

Fuhrer, J., Glette, K., Ivanovic, J., Larsson, P.G., Bekinschtein, T., Kochen, S., Knight, R.T., Tørresen, J., Solbakk, A.-K., Endestad, T., Blenkmann, A., 2021. Direct brain recordings reveal continuous encoding of structure in random stimuli. bioRxiv, o 14 .

Fyhn, M., Hafting, T., Treves, A., Moser, M.-B., Moser, E.I., 2007. Hippocampal remapping and grid realignment in entorhinal cortex. Nature 446, 190-194. https://doi.org/10.1038/nature05601

Gadian, D.G., Aicardi, J., Watkins, K.E., Porter, D.A., Mishkin, M., Vargha-Khadem, F., 2000. Developmental amnesia associated with early hypoxic-ischaemic injury. Brain 123, 499-507. https://doi.org/10.1093/brain/123.3.499

Gagnepain, P., Fauvel, B., Desgranges, B., Gaubert, M., Viader, F., Eustache, F., Groussard, M., Platel, H., 2017. Musical Expertise Increases Top-Down Modulation Over Hippocampal Activation during Familiarity Decisions. Frontiers in Human Neuroscience 11. https://doi.org/10.3389/fnhum.2017.00472

Gagnepain, P., Henson, R., Chételat, G., Desgranges, B., Lebreton, K., Eustache, F., 2011. Is Neocortical-Hippocampal Connectivity a Better Predictor of Subsequent Recollection than Local Increases in Hippocampal Activity? New Insights on the Role of Priming. Journal of Cognitive Neuroscience 23, 391-403. https://doi.org/10.1162/jocn.2010.21454

Gai, Z., Su, D., Wang, Y., Li, W., Cui, B., Li, K., She, X., Wang, R., 2017. Effects of chronic noise on the corticotropin-releasing factor system in the rat hippocampus: relevance to Alzheimer's disease-like tau hyperphosphorylation. Environmental Health and Preventive Medicine 22. https://doi.org/10.1186/s12199-017-0686-8

Galambos, R., Makeig, S., Talmachoff, P.J., 1981. A 40-Hz auditory potential recorded from the human scalp. Proceedings of the National Academy of Sciences 78, 2643-2647. https://doi.org/10.1073/pnas.78.4.2643

Gao, J., Wu, Y., Zhu, Z., Yang, C., Cheng, P., Liu, L., Sui, J., 2010. Neuronal firing activity of hippocampal pyramidal cells during an auditory discrimination task in conscious guinea pigs. Behavioural Brain Research 212, 35-40. https://doi.org/10.1016/j.bbr.2010.03.031

Garcia, A.D., Buffalo, E.A., 2020. Anatomy and Function of the Primate Entorhinal Cortex. Annual Review of Vision Science 6, 411-432. https://doi.org/10.1146/annurev-vision-030320-041115

Geerts, J.P., Chersi, F., Stachenfeld, K.L., Burgess, N., 2020. A general model of hippocampal and dorsal striatal learning and decision making. Proc Natl Acad Sci USA 117, 31427-31437. https://doi.org/10.1073/pnas.2007981117

Gelbard-Sagiv, H., Mukamel, R., Harel, M., Malach, R., Fried, I., 2008. Internally Generated Reactivation of Single Neurons in Human Hippocampus During Free Recall. Science 322, 96-101. https://doi.org/10.1126/science.1164685

Gershman, S.J., 2018. The Successor Representation: Its Computational Logic and Neural Substrates. The Journal of Neuroscience 38, 7193-7200. https://doi.org/10.1523/JNEUROSCI.0151-18.2018

Gilmartin, M.R., McEchron, M.D., 2005. Single Neurons in the Dentate Gyrus and CA1 of the Hippocampus Exhibit Inverse Patterns of Encoding During Trace Fear Conditioning. Behavioral Neuroscience 119, 164-179. https://doi.org/10.1037/0735-7044.119.1.164

Goble, T.J., Møller, A.R., Thompson, L.T., 2009. Acute high-intensity sound exposure alters responses of place cells in hippocampus. Hearing Research 253, 52-59. https://doi.org/10.1016/j.heares.2009.03.002

Golden, H.L., Agustus, J.L., Goll, J.C., Downey, L.E., Mummery, C.J., Schott, J.M., Crutch, S.J., Warren, J.D., 2015. Functional neuroanatomy of auditory scene analysis in Alzheimer's disease. NeuroImage: Clinical 7, 699-708. https://doi.org/10.1016/j.nicl.2015.02.019

Goldstein, L.H., Canavan, A.G.M., Polkey, C.E., 1988. Verbal and Abstract Designs Paired Associate Learning After Unilateral Temporal Lobectomy. Cortex 24, 41-52. https://doi.org/10.1016/S0010-9452(88)80016-9

Goll, J.C., Kim, L.G., Ridgway, G.R., Hailstone, J.C., Lehmann, M., Buckley, A.H., Crutch, S.J., Warren, J.D., 2012. Impairments of auditory scene analysis in Alzheimer's disease. Brain 135, 190-200. https://doi.org/10.1093/brain/awr260

Gonzalez-Perez, O., Chavez-Casillas, O., Jauregui-Huerta, F., Lopez-Virgen, V., Guzman-Muniz, J., Moy-Lopez, N., GonzalezCastaneda, R.E., Luquin, S., 2011. Stress by noise produces differential effects on the proliferation rate of radial astrocytes and survival of neuroblasts in the adult subgranular zone. Neuroscience Research 70, 243-250. https://doi.org/10.1016/j.neures.2011.03.013

Gosselin, N., 2006. Emotional responses to unpleasant music correlates with damage to the parahippocampal cortex. Brain 129, 2585-2592. https://doi.org/10.1093/brain/awl240 
Gosselin, N., Peretz, I., Hasboun, D., Baulac, M., Samson, S., 2011. Impaired recognition of musical emotions and facial expressions following anteromedial temporal lobe excision. Cortex 47, 1116-1125. https://doi.org/10.1016/j.cortex.2011.05.012

Gosselin, N., Peretz, I., Noulhiane, M., Hasboun, D., Beckett, C., Baulac, M., Samson, S., 2005. Impaired recognition of scary music following unilateral temporal lobe excision. Brain 128, 628-640. https://doi.org/10.1093/brain/awh420

Gottlieb, L.J., Uncapher, M.R., Rugg, M.D., 2010. Dissociation of the neural correlates of visual and auditory contextual encoding. Neuropsychologia 48, 137-144. https://doi.org/10.1016/j.neuropsychologia.2009.08.019

Graham, K.S., Barense, M.D., Lee, A.C.H., 2010. Going beyond LTM in the MTL: A synthesis of neuropsychological and neuroimaging findings on the role of the medial temporal lobe in memory and perception. Neuropsychologia 48, 831 853. https://doi.org/10.1016/j.neuropsychologia.2010.01.001

Grastyán, E., Lissák, K., Madarász, I., Donhoffer, H., 1959. Hippocampal electrical activity during the development of conditioned reflexes. Electroencephalography and Clinical Neurophysiology 11, 409-430. https://doi.org/10.1016/0013-4694(59)90040-9

Grau-Perales, A.B., Levy, E.R.J., Fenton, A.A., Gallo, M., 2019. Dorsal hippocampal damage disrupts the auditory contextdependent attenuation of taste neophobia in mice. Neurobiology of Learning and Memory 157, 121-127. https://doi.org/10.1016/j.nlm.2018.12.009

Green, J.D., Adey, W.R., 1956. Electrophysiological studies of hippocampal connections and excitability. Electroencephalography and Clinical Neurophysiology 8, 245-262. https://doi.org/10.1016/0013-4694(56)90117-1

Green, J.D., Arduini, A.A., 1954. Hippocampal electrical activity in arousal. Journal of Neurophysiology 17, 533-557. https://doi.org/10.1152/jn.1954.17.6.533

Griffiths, B.J., Fuentemilla, L., 2019. Event conjunction: How the hippocampus integrates episodic memories across event boundaries. Hippocampus. https://doi.org/10.1002/hipo.23161

Griffiths, T.D., Lad, M., Kumar, S., Holmes, E., McMurray, B., Maguire, E.A., Billig, A.J., Sedley, W., 2020. How Can Hearing Loss Cause Dementia? Neuron 108, 401-412. https://doi.org/10.1016/j.neuron.2020.08.003

Griffiths, T.D., Warren, J.D., 2004. What is an auditory object? Nature Reviews Neuroscience 5, 887-892. https://doi.org/10.1038/nrn1538

Groussard, M., La Joie, R., Rauchs, G., Landeau, B., Chételat, G., Viader, F., Desgranges, B., Eustache, F., Platel, H., 2010 a. When Music and Long-Term Memory Interact: Effects of Musical Expertise on Functional and Structural Plasticity in the Hippocampus. PLoS ONE 5, e13225. https://doi.org/10.1371/journal.pone.0013225

Groussard, M., Rauchs, G., Landeau, B., Viader, F., Desgranges, B., Eustache, F., Platel, H., 2010b. The neural substrates of musical memory revealed by fMRI and two semantic tasks. NeuroImage 53, 1301-1309. https://doi.org/10.1016/j.neuroimage.2010.07.013

Groussard, M., Viader, F., Landeau, B., Desgranges, B., Eustache, F., Platel, H., 2014. The effects of musical practice on structural plasticity: The dynamics of grey matter changes. Brain and Cognition 90, 174-180. https://doi.org/10.1016/j.bandc.2014.06.013

Gruart, A., Leal-Campanario, R., López-Ramos, J.C., Delgado-García, J.M., 2015. Functional basis of associative learning and its relationships with long-term potentiation evoked in the involved neural circuits: Lessons from studies in behaving mammals. Neurobiology of Learning and Memory 124, 3-18. https://doi.org/10.1016/j.nlm.2015.04.006

Gunbey, H.P., Gunbey, E., Aslan, K., Bulut, T., Unal, A., Incesu, L., 2015. Limbic-Auditory Interactions of Tinnitus: An Evaluation Using Diffusion Tensor Imaging. Clinical Neuroradiology 27, 221-230. https://doi.org/10.1007/s00062 015-0473-0

Hafting, T., Fyhn, M., Molden, S., Moser, M.-B., Moser, E.I., 2005. Microstructure of a spatial map in the entorhinal cortex. Nature 436, 801-806. https://doi.org/10.1038/nature03721

Haider, S., Naqvi, F., Batool, Z., Tabassum, S., Perveen, T., Saleem, S., Haleem, D.H., 2012. Decreased Hippocampal 5-HT and DA Levels Following Sub-Chronic Exposure to Noise Stress: Impairment in both Spatial and Recognition Memory in Male Rats. Scientia Pharmaceutica 80, 1001-1011. https://doi.org/10.3797/scipharm.1207-15

Halgren, E., Baudena, P., Clarke, J.M., Heit, G., Marinkovic, K., Devaux, B., Vignal, J.-P., Biraben, A., 1995. Intracerebral potentials to rare target and distractor auditory and visual stimuli. II. Medial, lateral and posterior temporal lobe. Electroencephalography and Clinical Neurophysiology 94, 229-250. https://doi.org/10.1016/0013-4694(95)98475-N

Halgren, E., Squires, N., Wilson, C., Rohrbaugh, J., Babb, T., Crandall, P., 1980. Endogenous potentials generated in the human hippocampal formation and amygdala by infrequent events. Science 210, 803-805. https://doi.org/10.1126/science. 7434000

Hall, R.D., Borbely, A.A., 1970. Acoustically evoked potentials in the rat during sleep and waking. Experimental Brain Research 11. https://doi.org/10.1007/BF00234203

Hallam, R.S., Mckenna, L., Shurlock, L., 2004. Tinnitus impairs cognitive efficiency. International Journal of Audiology 43, 218 226. https://doi.org/10.1080/14992020400050030

Han, M.W., Ahn, J.H., Kang, J.K., Lee, E.M., Lee, J.H., Bae, J.H., Chung, J.W., 2011. Central auditory processing impairment in patients with temporal lobe epilepsy. Epilepsy \& Behavior 20, 370-374. https://doi.org/10.1016/j.yebeh.2010.12.032

Hannula, D.E., Ranganath, C., 2008. Medial Temporal Lobe Activity Predicts Successful Relational Memory Binding. Journal of Neuroscience 28, 116-124. https://doi.org/10.1523/JNEUROSCI.3086-07.2008

Hardy, C.J.D., Yong, K.X.X., Goll, J.C., Crutch, S.J., Warren, J.D., 2020. Impairments of auditory scene analysis in posterior cortical atrophy. Brain 143, 2689-2695. https://doi.org/10.1093/brain/awaa221

Harrington, M.O., Cairney, S.A., 2021. Sounding It Out: Auditory Stimulation and Overnight Memory Processing. Curr Sleep Medicine Rep. https://doi.org/10.1007/s40675-021-00207-0

Hassabis, D., Kumaran, D., Vann, S.D., Maguire, E.A., 2007. Patients with hippocampal amnesia cannot imagine new experiences. Proceedings of the National Academy of Sciences 104, 1726-1731. https://doi.org/10.1073/pnas.0610561104

Hasselmo, M.E., Wyble, B.P., 1997. Free recall and recognition in a network model of the hippocampus: simulating effects of 
scopolamine on human memory function. Behavioural Brain Research 89, 1-34. https://doi.org/10.1016/S01664328(97)00048-X

Hattori, M., Onoda, K., Sakata, S., 2010. Identification of rat P3-like processes in the anterior cingulate cortex and hippocampus. Neuroscience Letters 472, 43-46. https://doi.org/10.1016/j.neulet.2010.01.052

Hebscher, M., Wing, E., Ryan, J., Gilboa, A., 2019. Rapid Cortical Plasticity Supports Long-Term Memory Formation. Trends in Cognitive Sciences 23, 989-1002. https://doi.org/10.1016/j.tics.2019.09.009

Heinrich, A., Carlyon, R.P., Davis, M.H., Johnsrude, I.S., 2008. Illusory Vowels Resulting from Perceptual Continuity: A Functional Magnetic Resonance Imaging Study. Journal of Cognitive Neuroscience 20, 1737-1752. https://doi.org/10.1162/jocn.2008.20069

Heit, G., Smith, M.E., Halgren, E., 1990. Neuronal activity in the human medial temporal lobe during recognition memory. Brain 113, 1093-1112. https://doi.org/10.1093/brain/113.4.1093

Helmstaedter, C., Elger, C.E., 1996. Cognitive Consequences of Two-Thirds Anterior Temporal Lobectomy on Verbal Memory in 144 Patients: A Three-Month Follow-Up Study. Epilepsia 37, 171-180. https://doi.org/10.1111/j.15281157.1996.tb00009.x

Helmstaedter, C., Grunwald, Th., Lehnertz, K., Gleißner, U., Elger, C.E., 1997. Differential Involvement of Left Temporolateral and Temporomesial Structures in Verbal Declarative Learning and Memory: Evidence from Temporal Lobe Epilepsy. Brain and Cognition 35, 110-131. https://doi.org/10.1006/brcg.1997.0930

Henin, S., Turk-Browne, N.B., Friedman, D., Liu, A., Dugan, P., Flinker, A., Doyle, W., Devinsky, O., Melloni, L., 2021. Learning hierarchical sequence representations across human cortex and hippocampus. Sci. Adv. 7, eabc4530. https://doi.org/10.1126/sciadv.abc4530

Herdener, M., Esposito, F., di Salle, F., Boller, C., Hilti, C.C., Habermeyer, B., Scheffler, K., Wetzel, S., Seifritz, E., CattapanLudewig, K., 2010. Musical Training Induces Functional Plasticity in Human Hippocampus. Journal of Neuroscience 30, 1377-1384. https://doi.org/10.1523/JNEUROSCI.4513-09.2010

Herholz, S.C., Coffey, E.B.J., Pantev, C., Zatorre, R.J., 2016. Dissociation of Neural Networks for Predisposition and for Training-Related Plasticity in Auditory-Motor Learning. Cerebral Cortex 26, 3125-3134. https://doi.org/10.1093/cercor/bhv138

Hill, R.M., Boto, E., Rea, M., Holmes, N., Leggett, J., Coles, L.A., Papastavrou, M., Everton, S.K., Hunt, B.A.E., Sims, D., Osborne, J., Shah, V., Bowtell, R., Brookes, M.J., 2020. Multi-channel whole-head OPM-MEG: Helmet design and a comparison with a conventional system. NeuroImage 219, 116995. https://doi.org/10.1016/j.neuroimage.2020.116995

Hirano, T., Yamaguchi, M., 1985. Hippocampal unit response during temporal single alternation of classical conditioning with rewarding brain stimulation in the rat. Physiological Psychology 13, 7-14. https://doi.org/10.3758/BF03326488

Hirano, Y., Fujita, M., Watanabe, K., Niwa, M., Takahashi, T., Kanematsu, M., Ido, Y., Tomida, M., Onozuka, M., 2006. Effect of unpleasant loud noise on hippocampal activities during picture encoding: An fMRI study. Brain and Cognition 61, 280-285. https://doi.org/10.1016/j.bandc.2006.02.003

Hoefeijzers, S., Dewar, M., Della Sala, S., Zeman, A., Butler, C., 2013. Accelerated long-term forgetting in transient epileptic amnesia: An acquisition or consolidation deficit? Neuropsychologia 51, 1549-1555. https://doi.org/10.1016/j.neuropsychologia.2013.04.017

Hoehler, F.K., Thompson, R.F., 1979. The effect of temporal single alternation on learned increases in hippocampal unit activity in classical conditioning of the rabbit nictitating membrane response. Physiological Psychology 7, 345-351. https://doi.org/10.3758/BF03326655

Hoffman, R.E., Anderson, A.W., Varanko, M., Gore, J.C., Hampson, M., 2008. Time course of regional brain activation associated with onset of auditory/verbal hallucinations. British Journal of Psychiatry 193, 424-425. https://doi.org/10.1192/bjp.bp.107.040501

Hoffmann, L.C., Cicchese, J.J., Berry, S.D., 2015. Harnessing the power of theta: natural manipulations of cognitive performance during hippocampal theta-contingent eyeblink conditioning. Frontiers in Systems Neuroscience 9, 50. https://doi.org/10.3389/fnsys.2015.00050

Hollup, S.A., Molden, S., Donnett, J.G., Moser, M.-B., Moser, E.I., 2001. Accumulation of Hippocampal Place Fields at the Goal Location in an Annular Watermaze Task. The Journal of Neuroscience 21, 1635-1644. https://doi.org/10.1523/JNEUROSCI.21-05-01635.2001

Holmes, S.D., Roberts, B., 2011. The influence of adaptation and inhibition on the effects of onset asynchrony on auditory grouping. Journal of Experimental Psychology: Human Perception and Performance 37, 1988-2000. https://doi.org/10.1037/a0025642

Holt, W., Maren, S., 1999. Muscimol Inactivation of the Dorsal Hippocampus Impairs Contextual Retrieval of Fear Memory. J. Neurosci. 19, 9054-9062. https://doi.org/10.1523/JNEUROSCI.19-20-09054.1999

Hosseini-Sharifabad, M., Sabahi, A., 2008. Exposure to chronic noise reduces the volume of hippocampal subregions in rats. Iranian Journal of Basic Medical Sciences 11, 18-24.

Howard, M.W., Fotedar, M.S., Datey, A.V., Hasselmo, M.E., 2005. The Temporal Context Model in Spatial Navigation and Relational Learning: Toward a Common Explanation of Medial Temporal Lobe Function Across Domains. Psychological Review 112, 75-116. https://doi.org/10.1037/0033-295X.112.1.75

Hu, L., Yang, J., Song, T., Hou, N., Liu, Y., Zhao, X., Zhang, D., Wang, L., Wang, T., Huang, C., 2014. A new stress model, a scream sound, alters learning and monoamine levels in rat brain. Physiology \& Behavior 123, 105-113. https://doi.org/10.1016/j.physbeh.2013.09.010

Huet-Bello, O., Ruvalcaba-Delgadillo, Y., Feria-Velasco, A., Gonzalez-Castaneda, R.E., Garcia-Estrada, J., Macias-Islas, M.A., Jauregui-Huerta, F., Luquin, S., 2017. Environmental noise exposure modifies astrocyte morphology in hippocampus of young male rats. Noise and Health 19, 239-244.

Huijgen, J., Dellacherie, D., Tillmann, B., Clément, S., Bigand, E., Dupont, S., Samson, S., 2015. The feeling of familiarity for music in patients with a unilateral temporal lobe lesion: A gating study. Neuropsychologia 77, 313-320. 
https://doi.org/10.1016/j.neuropsychologia.2015.09.007

Hunsaker, M.R., Kesner, R.P., 2008. Dissociations across the dorsal-ventral axis of CA3 and CA1 for encoding and retrieval of contextual and auditory-cued fear. Neurobiology of Learning and Memory 89, 61-69. https://doi.org/10.1016/j.nlm.2007.08.016

Hunter, P.G., Schellenberg, E.G., Schimmack, U., 2010. Feelings and perceptions of happiness and sadness induced by music: Similarities, differences, and mixed emotions. Psychology of Aesthetics, Creativity, and the Arts 4, 47-56. https://doi.org/10.1037/a0016873

Hwang, J.-H., Chou, P.-H., Wu, C.-W., Chen, J.-H., Liu, T.-C., 2009. Brain activation in patients with idiopathic hyperacusis. American Journal of Otolaryngology 30, 432-434. https://doi.org/10.1016/j.amjoto.2008.08.005

Insausti, R., Amaral, D.G., Cowan, W.M., 1987. The entorhinal cortex of the monkey: III. Subcortical afferents. The Journal of Comparative Neurology 264, 396-408. https://doi.org/10.1002/cne.902640307

Inta, D., Vogt, M.A., Elkin, H., Weber, T., Lima-Ojeda, J.M., Schneider, M., Luoni, A., Riva, M.A., Gertz, K., Hellmann-Regen, J., Kronenberg, G., Meyer-Lindenberg, A., Sprengel, R., Gass, P., 2014. Phenotype of mice with inducible ablation of GluA1 AMPA receptors during late adolescence: Relevance for mental disorders: BEHAVIORAL CHANGES FOLLOWING ADOLESCENT GLUA1 ABLATION. Hippocampus 24, 424-435. https://doi.org/10.1002/hipo.22236

Iordanova, M.D., Burnett, D.J., Aggleton, J.P., Good, M., Honey, R.C., 2009. The role of the hippocampus in mnemonic integration and retrieval: complementary evidence from lesion and inactivation studies. European Journal of Neuroscience 30, 2177-2189. https://doi.org/10.1111/j.1460-9568.2009.07010.x

Irmiš, F., Radil-Weiss, T., Lát, J., Krekule, I., 1970. Inter-individual differences in hippocampal theta activity during habituation. Electroencephalography and Clinical Neurophysiology 28, 24-31. https://doi.org/10.1016/0013-4694(70)90004-0

Itskov, P.M., Vinnik, E., Honey, C., Schnupp, J., Diamond, M.E., 2012. Sound sensitivity of neurons in rat hippocampus during performance of a sound-guided task. Journal of Neurophysiology 107, 1822-1834. https://doi.org/10.1152/jn.00404.2011

Jablonowski, J., Taesler, P., Fu, Q., Rose, M., 2018. Implicit acoustic sequence learning recruits the hippocampus. PLOS ONE 13, e0209590. https://doi.org/10.1371/journal.pone.0209590

Jackson, J., Redish, A.D., 2007. Network dynamics of hippocampal cell-assemblies resemble multiple spatial maps within single tasks. Hippocampus 17, 1209-1229. https://doi.org/10.1002/hipo.20359

Jacobs, J., Weidemann, C.T., Miller, J.F., Solway, A., Burke, J.F., Wei, X.-X., Suthana, N., Sperling, M.R., Sharan, A.D., Fried, I., Kahana, M.J., 2013. Direct recordings of grid-like neuronal activity in human spatial navigation. Nat Neurosci 16, 1188-1190. https://doi.org/10.1038/nn.3466

Jacobsen, J.-H., Stelzer, J., Fritz, T.H., Chételat, G., La Joie, R., Turner, R., 2015. Why musical memory can be preserved in advanced Alzheimer's disease. Brain 138, 2438-2450. https://doi.org/10.1093/brain/awv135

Jafari, Z., Okuma, M., Karem, H., Mehla, J., Kolb, B.E., Mohajerani, M.H., 2019. Prenatal noise stress aggravates cognitive decline and the onset and progression of beta amyloid pathology in a mouse model of Alzheimer's disease. Neurobiology of Aging 77, 66-86. https://doi.org/10.1016/j.neurobiolaging.2019.01.019

Jafarpour, A., Piai, V., Lin, J.J., Knight, R.T., 2017. Human hippocampal pre-activation predicts behavior. Scientific Reports 7. https://doi.org/10.1038/s41598-017-06477-5

James, C.E., Britz, J., Vuilleumier, P., Hauert, C.-A., Michel, C.M., 2008. Early neuronal responses in right limbic structures mediate harmony incongruity processing in musical experts. NeuroImage 42, 1597-1608. https://doi.org/10.1016/j.neuroimage.2008.06.025

Janata, P., 2002. The Cortical Topography of Tonal Structures Underlying Western Music. Science 298, 2167-2170. https://doi.org/10.1126/science.1076262

Jang, S.H., Choi, E.B., 2022. Evaluation of Structural Neural Connectivity Between the Primary Auditory Cortex and CognitionRelated Brain Areas Using Diffusion Tensor Tractography in 43 Normal Adults. Med Sci Monit 28. https://doi.org/10.12659/MSM.936131

Jardri, R., Lucas, B., Delevoye-Turrell, Y., Delmaire, C., Delion, P., Thomas, P., Goeb, J.-L., 2007. An 11-year-old boy with drugresistant schizophrenia treated with temporo-parietal rTMS. Molecular Psychiatry 12, 320-320. https://doi.org/10.1038/sj.mp.4001968

Jardri, R., Pins, D., Bubrovszky, M., Lucas, B., Lethuc, V., Delmaire, C., Vantyghem, V., Despretz, P., Thomas, P., 2009. Neural functional organization of hallucinations in schizophrenia: Multisensory dissolution of pathological emergence in consciousness. Consciousness and Cognition 18, 449-457. https://doi.org/10.1016/j.concog.2008.12.009

Jardri, R., Pouchet, A., Pins, D., Thomas, P., 2011. Cortical Activations During Auditory Verbal Hallucinations in Schizophrenia: A Coordinate-Based Meta-Analysis. American Journal of Psychiatry 168, 73-81. https://doi.org/10.1176/appi.ajp.2010.09101522

Jastreboff, P.J., 1990. Phantom auditory perception (tinnitus): mechanisms of generation and perception. Neuroscience Research 8, 221-254. https://doi.org/10.1016/0168-0102(90)90031-9

Jáuregui-Huerta, F., García-Estrada, J., Ruvalcaba-Delgadillo, Y., Trujillo, X., Huerta, M., Feria-Velasco, A., Gonzalez-Perez, O., Luquín, S., 2011. Chronic exposure of juvenile rats to environmental noise impairs hippocampal cell proliferation in adulthood. Noise and Health 13, 286. https://doi.org/10.4103/1463-1741.82961

Jayakar, R., King, T.Z., Morris, R., Na, S., 2015. Hippocampal volume and auditory attention on a verbal memory task with adult survivors of pediatric brain tumor. Neuropsychology 29, 303-319. https://doi.org/10.1037/neu0000183

Jeneson, A., Squire, L.R., 2011. Working memory, long-term memory, and medial temporal lobe function. Learning \& Memory 19, 15-25. https://doi.org/10.1101/lm.024018.111

Jin, J., Maren, S., 2015. Prefrontal-Hippocampal Interactions in Memory and Emotion. Frontiers in Systems Neuroscience 9, 170. https://doi.org/10.3389/fnsys.2015.00170

Jin, S.G., Kim, M.J., Park, S.Y., Park, S.N., 2017. Stress hormonal changes in the brain and plasma after acute noise exposure in mice. Auris Nasus Larynx 44, 272-276. https://doi.org/10.1016/j.anl.2016.07.013 
Joassin, F., Pesenti, M., Maurage, P., Verreckt, E., Bruyer, R., Campanella, S., 2011. Cross-modal interactions between human faces and voices involved in person recognition. Cortex 47, 367-376. https://doi.org/10.1016/j.cortex.2010.03.003

Joris, P.X., Schreiner, C.E., Rees, A., 2004. Neural Processing of Amplitude-Modulated Sounds. Physiological Reviews 84, 541577. https://doi.org/10.1152/physrev.00029.2003

Julian, J.B., Keinath, A.T., Frazzetta, G., Epstein, R.A., 2018. Human entorhinal cortex represents visual space using a boundaryanchored grid. Nat Neurosci 21, 191-194. https://doi.org/10.1038/s41593-017-0049-1

Jung, R., Kornmüller, A.E., 1938. Eine Methodik der Ableitung Iokalisierter Potentialschwankungen aus subcorticalen Hirngebieten. Archiv für Psychiatrie und Nervenkrankheiten 109, 1-30. https://doi.org/10.1007/BF02157817

Kahn, I., Andrews-Hanna, J.R., Vincent, J.L., Snyder, A.Z., Buckner, R.L., 2008. Distinct Cortical Anatomy Linked to Subregions of the Medial Temporal Lobe Revealed by Intrinsic Functional Connectivity. Journal of Neurophysiology 100, 129139. https://doi.org/10.1152/jn.00077.2008

Kaifosh, P., Lovett-Barron, M., Turi, G.F., Reardon, T.R., Losonczy, A., 2013. Septo-hippocampal GABAergic signaling across multiple modalities in awake mice. Nature Neuroscience 16, 1182-1184. https://doi.org/10.1038/nn.3482

Kalm, K., Davis, M.H., Norris, D., 2013. Individual Sequence Representations in the Medial Temporal Lobe. Journal of Cognitive Neuroscience 25, 1111-1121. https://doi.org/10.1162/jocn_a_00378

Kamiński, J., Sullivan, S., Chung, J.M., Ross, I.B., Mamelak, A.N., Rutishauser, U., 2017. Persistently active neurons in human medial frontal and medial temporal lobe support working memory. Nature Neuroscience 20, 590-601. https://doi.org/10.1038/nn.4509

Kaneko, W.M., Riley, E.P., Ehlers, C.L., 1993. Electrophysiological and behavioral findings in rats prenatally exposed to alcohol. Alcohol 10, 169-178. https://doi.org/10.1016/0741-8329(93)90099-A

Kapolowicz, M.R., Thompson, L.T., 2016. Acute high-intensity noise induces rapid Arc protein expression but fails to rapidly change GAD expression in amygdala and hippocampus of rats: Effects of treatment with D-cycloserine. Hearing Research 342, 69-79. https://doi.org/10.1016/j.heares.2016.09.010

Kato, T., Erhard, P., Takayama, Y., Strupp, J., Le, T.H., Ogawa, S., Ugurbil, K., 1998. Human hippocampal long-term sustained response during word memory processing. NeuroReport 9, 1041-1047.

Keane, M.M., Gabrieli, J.D.E., Mapstone, H.C., Johnson, K.A., Corkin, S., 1995. Double dissociation of memory capacities after bilateral occipital-lobe or medial temporal-lobe lesions. Brain 118, 1129-1148. https://doi.org/10.1093/brain/118.5.1129

Kemble, E.D., Ison, J.R., 1971. Limbic lesions and the inhibition of startle reactions in the rat by conditions of preliminary stimulation. Physiology \& Behavior 7, 925-928. https://doi.org/10.1016/0031-9384(71)90068-0

Khalfa, S., Guye, M., Peretz, I., Chapon, F., Girard, N., Chauvel, P., Liégeois-Chauvel, C., 2008. Evidence of lateralized anteromedial temporal structures involvement in musical emotion processing. Neuropsychologia 46, 2485-2493. https://doi.org/10.1016/j.neuropsychologia.2008.04.009

Khan, U.A., Liu, L., Provenzano, F.A., Berman, D.E., Profaci, C.P., Sloan, R., Mayeux, R., Duff, K.E., Small, S.A., 2014. Molecular drivers and cortical spread of lateral entorhinal cortex dysfunction in preclinical Alzheimer's disease. Nat Neurosci 17, 304-311. https://doi.org/10.1038/nn.3606

Killian, N.J., Jutras, M.J., Buffalo, E.A., 2012. A map of visual space in the primate entorhinal cortex. Nature 491, 761-764. https://doi.org/10.1038/nature11587

Kim, B.-K., Ko, I.-G., Kim, S.-E., Kim, C.-J., Yoon, J.-S., Baik, H.-H., Jin, B.-K., Lee, C.-Y., Baek, S.-B., Shin, M.-S., 2013. Impact of Several Types of Stresses on Short-term Memory and Apoptosis in the Hippocampus of Rats. Int Neurourol J 17, 114-120. https://doi.org/10.5213/inj.2013.17.3.114

Kim, H., Lee, M.-H., Chang, H.-K., Lee, T.-H., Lee, H.-H., Shin, M.-C., Shin, M.-S., Won, R., Shin, H.-S., Kim, C.-J., 2006. Influence of prenatal noise and music on the spatial memory and neurogenesis in the hippocampus of developing rats. Brain and Development 28, 109-114. https://doi.org/10.1016/j.braindev.2005.05.008

Kim, J.J., Diamond, D.M., 2002. The stressed hippocampus, synaptic plasticity and lost memories. Nature Reviews Neuroscience 3, 453-462. https://doi.org/10.1038/nrn849

Kim, T.-W., Shin, M.-S., Park, J.-K., Shin, M.-A., Lee, H.-H., Lee, S.-J., 2013. Treadmill exercise alleviates prenatal noise stressinduced impairment of spatial learning ability through enhancing hippocampal neurogenesis in rat pups. Journal of Exercise Rehabilitation 9, 451-456. https://doi.org/10.12965/jer.130064

Kimble, D.P., 1968. Hippocampus and internal inhibition. Psychological Bulletin 70, 285-295. https://doi.org/10.1037/h0026470

Kirste, I., Nicola, Z., Kronenberg, G., Walker, T.L., Liu, R.C., Kempermann, G., 2015. Is silence golden? Effects of auditory stimuli and their absence on adult hippocampal neurogenesis. Brain Structure and Function 220, 1221-1228. https://doi.org/10.1007/s00429-013-0679-3

Klee, J.L., Souza, B.C., Battaglia, F.P., 2021. Learning differentially shapes prefrontal and hippocampal activity during classical conditioning. eLife 10, e65456. https://doi.org/10.7554/eLife.65456

Kliuchko, M., Puoliväli, T., Heinonen-Guzejev, M., Tervaniemi, M., Toiviainen, P., Sams, M., Brattico, E., 2018. Neuroanatomical substrate of noise sensitivity. NeuroImage 167, 309-315. https://doi.org/10.1016/j.neuroimage.2017.11.041

Knierim, J.J., 2015. The hippocampus. Current Biology 25, R1116-R1121. https://doi.org/10.1016/j.cub.2015.10.049

Knösche, T.R., Neuhaus, C., Haueisen, J., Alter, K., Maess, B., Witte, O.W., Friederici, A.D., 2005. Perception of phrase structure in music: Perception of Phrase Structure in Music. Human Brain Mapping 24, 259-273. https://doi.org/10.1002/hbm.20088

Koelsch, S., Fritz, T., v. Cramon, D.Y., Müller, K., Friederici, A.D., 2006. Investigating emotion with music: An fMRI study. Human Brain Mapping 27, 239-250. https://doi.org/10.1002/hbm.20180

Koelsch, S., Remppis, A., Sammler, D., Jentschke, S., Mietchen, D., Fritz, T., Bonnemeier, H., Siebel, W.A., 2007. A cardiac signature of emotionality: A cardiac signature of emotionality. European Journal of Neuroscience 26, 3328-3338. https://doi.org/10.1111/j.1460-9568.2007.05889.x 
Koelsch, S., Skouras, S., 2014. Functional centrality of amygdala, striatum and hypothalamus in a "small-world" network underlying joy: An fMRI study with music: A Neural Network Underlying Joy. Human Brain Mapping 35, 3485-3498. https://doi.org/10.1002/hbm.22416

Koelsch, S., Skouras, S., Fritz, T., Herrera, P., Bonhage, C., Küssner, M.B., Jacobs, A.M., 2013. The roles of superficial amygdala and auditory cortex in music-evoked fear and joy. NeuroImage 81, 49-60. https://doi.org/10.1016/i.neuroimage.2013.05.008

Koelsch, S., Skouras, S., Lohmann, G., 2018. The auditory cortex hosts network nodes influential for emotion processing: An fMRI study on music-evoked fear and joy. PLOS ONE 13, e0190057. https://doi.org/10.1371/journal.pone.0190057

Koike, A., Ishijima, B., 1996. Preserved musical abilities following right temporal lobectomy. J. Neurosurg. 85, 5.

Kok, P., Rait, L.I., Turk-Browne, N.B., 2020. Content-based Dissociation of Hippocampal Involvement in Prediction. Journal of Cognitive Neuroscience 32, 527-545. https://doi.org/10.1162/jocn_a_01509

Kok, P., Turk-Browne, N.B., 2018. Associative Prediction of Visual Shape in the Hippocampus. The Journal of Neuroscience 38, 6888-6899. https://doi.org/10.1523/JNEUROSCI.0163-18.2018

Korb, E., Finkbeiner, S., 2011. Arc in synaptic plasticity: from gene to behavior. Trends in Neurosciences 34, 591-598. https://doi.org/10.1016/j.tins.2011.08.007

Kornblith, S., Quian Quiroga, R., Koch, C., Fried, I., Mormann, F., 2017. Persistent Single-Neuron Activity during Working Memory in the Human Medial Temporal Lobe. Current Biology 27, 1026-1032. https://doi.org/10.1016/j.cub.2017.02.013

Kotelenko, L.M., Nikitin, N.I., Altman, Ya.A., 2013. Estimation by humans of signals simulating different sound movement directions and specificity of the perception of these signals by patients with temporal epilepsy. Human Physiology 39, 241-247. https://doi.org/10.1134/S0362119713030122

Kotz, S.A., Kalberlah, C., Bahlmann, J., Friederici, A.D., Haynes, J.-D., 2013. Predicting vocal emotion expressions from the human brain. Human Brain Mapping 34, 1971-1981. https://doi.org/10.1002/hbm.22041

Kowalska, D.M., 1999. Effects of the anterior temporal lobe lesions, separate or combined with hippocampal damage, on spatial delayed responses guided by auditory stimulus. Acta Neurobiol Exp (Wars) 59, 303-313.

Kowalska, D.M., Kuśmierek, P., Kosmal, A., Mishkin, M., 2001. Neither perirhinal/entorhinal nor hippocampal lesions impair short-term auditory recognition memory in dogs. Neuroscience 104, 965-978. https://doi.org/10.1016/S03064522(01)00140-3

Kragel, J.E., Schuele, S., VanHaerents, S., Rosenow, J.M., Voss, J.L., 2021. Rapid coordination of effective learning by the human hippocampus. Science Advances 7.

Kraus, K.S., Canlon, B., 2012. Neuronal connectivity and interactions between the auditory and limbic systems. Effects of noise and tinnitus. Hearing Research 288, 34-46. https://doi.org/10.1016/j.heares.2012.02.009

Kraus, K.S., Mitra, S., Jimenez, Z., Hinduja, S., Ding, D., Jiang, H., Gray, L., Lobarinas, E., Sun, W., Salvi, R.J., 2010. Noise trauma impairs neurogenesis in the rat hippocampus. Neuroscience 167, 1216-1226. https://doi.org/10.1016/j.neuroscience.2010.02.071

Kreyberg, S., Torvik, A., Bjørneboe, A., Wilk-Larsen, W., Jacobsen, D., 1992. Trimethyltin poisoning: report of a case with postmortem examination. Clinical Neuropathology 11, 256-259.

Kropotov, J.D., Alho, K., Näätänen, R., Ponomarev, V.A., Kropotova, O.V., Anichkov, A.D., Nechaev, V.B., 2000. Human auditory-cortex mechanisms of preattentive sound discrimination. Neuroscience Letters 280, 87-90. https://doi.org/10.1016/S0304-3940(00)00765-5

Kropotov, J.D., Naatanen, R., Sevostianov, A.V., Alho, K., Reinikainen, K., Kropotova, O.V., 1995. Mismatch negativity to auditory stimulus change recorded directly from the human temporal cortex. Psychophysiology 32, 418-422.

Kubit, B., Janata, P., 2018. Listening for memories: Attentional focus dissociates functional brain networks engaged by memoryevoking music. Psychomusicology: Music, Mind, and Brain 28, 82-100. https://doi.org/10.1037/pmu0000210

Kubovy, M., Van Valkenburg, D., 2001. Auditory and visual objects. Cognition 80, 97-126. https://doi.org/10.1016/S00100277(00)00155-4

Kumar, S., Bonnici, H.M., Teki, S., Agus, T.R., Pressnitzer, D., Maguire, E.A., Griffiths, T.D., 2014. Representations of specific acoustic patterns in the auditory cortex and hippocampus. Proceedings of the Royal Society B: Biological Sciences 281 , 20141000-20141000. https://doi.org/10.1098/rspb.2014.1000

Kumar, S., Gander, P.E., Berger, J.I., Billig, A.J., Nourski, K.V., Oya, H., Kawasaki, H., Howard, M.A., Griffiths, T.D., 2021. Oscillatory correlates of auditory working memory examined with human electrocorticography. Neuropscyhologia 150. https://doi.org/10.1101/2020.06.19.161901

Kumar, S., Joseph, S., Gander, P.E., Barascud, N., Halpern, A.R., Griffiths, T.D., 2016. A Brain System for Auditory Working Memory. Journal of Neuroscience 36, 4492-4505. https://doi.org/10.1523/JNEUROSCI.4341-14.2016

Kumar, S., Stephan, K.E., Warren, J.D., Friston, K.J., Griffiths, T.D., 2007. Hierarchical Processing of Auditory Objects in Humans. PLoS Computational Biology 3, 9.

Kumar, S., Tansley-Hancock, O., Sedley, W., Winston, J.S., Callaghan, M.F., Allen, M., Cope, T.E., Gander, P.E., Bamiou, D.-E., Griffiths, T.D., 2017. The Brain Basis for Misophonia. Current Biology 27, 527-533. https://doi.org/10.1016/j.cub.2016.12.048

Kumaran, D., Maguire, E.A., 2007. Which computational mechanisms operate in the hippocampus during novelty detection? Hippocampus 17, 735-748. https://doi.org/10.1002/hipo.20326

Kurczek, J., Brown-Schmidt, S., Duff, M., 2013. Hippocampal contributions to language: Evidence of referential processing deficits in amnesia. Journal of Experimental Psychology: General 142, 1346-1354. https://doi.org/10.1037/a0034026

Kurioka, T., Mogi, S., Yamashita, T., 2021. Decreasing auditory input induces neurogenesis impairment in the hippocampus. Sci Rep 11, 423. https://doi.org/10.1038/s41598-020-80218-z

Lad, M., Mullally, S.L., Houston, A.L., Kelly, T., Griffiths, T.D., 2019. Characterizing memory loss in patients with autoimmune limbic encephalitis hippocampal lesions. Hippocampus 29, 1114-1120. https://doi.org/10.1002/hipo.23150

Lai, H., 1988. Effects of repeated exposure to white noise on central cholinergic activity in the rat. Brain Research 442, 403-406. 
https://doi.org/10.1016/0006-8993(88)91535-1

Lai, H., 1987. Acute exposure to noise affects sodium-dependent high-affinity choline uptake in the central nervous system of the rat. Pharmacology Biochemistry and Behavior 28, 147-151. https://doi.org/10.1016/0091-3057(87)90205-X

Lai, H., Carino, M.A., 1990. Acute White Noise Exposure Affects the Concentration of Benzodiazepine Receptors in the Brain of the Rat. Pharmacology Biochemistry and Behavior 36, 985-987.

Lai, H., Carino, M.A., Wen, Y.-F., 1989. Repeated noise exposure affects muscarinic cholinergic receptors in the rat brain. Brain Research 488, 361-364. https://doi.org/10.1016/0006-8993(89)90731-2

Lan, L., Chen, Y.-C., Shang, S., Lu, L., Xu, J.-J., Yin, X., Wu, Y., Cai, Y., 2022. Topological features of limbic dysfunction in chronicity of tinnitus with intact hearing: New hypothesis for 'noise-cancellation' mechanism. Progress in NeuroPsychopharmacology and Biological Psychiatry 113, 110459. https://doi.org/10.1016/j.pnpbp.2021.110459

Lancelot, C., Ahad, P., Noulhiane, M., Hasboun, D., Baulac, M., Samson, S., 2005. Loss of memory for auditory-spatial associations following unilateral medial temporal-lobe damage. Neuropsychologia 43, 1975-1982. https://doi.org/10.1016/i.neuropsychologia.2005.03.006

Lancelot, C., Samson, S., Ahad, P., Baulac, M., 2003. Effect of unilateral temporal lobe resection on short-term memory for auditory object and sound location. Annals of the New York Academy of Sciences 999, 377-380.

Landgrebe, M., Langguth, B., Rosengarth, K., Braun, S., Koch, A., Kleinjung, T., May, A., de Ridder, D., Hajak, G., 2009. Structural brain changes in tinnitus: Grey matter decrease in auditory and non-auditory brain areas. NeuroImage 46, 213-218. https://doi.org/10.1016/j.neuroimage.2009.01.069

Lane, R.D., Reiman, E.M., Bradley, M.M., Lang, P.J., Ahern, G.L., Davidson, R.J., Schwartz, G.E., 1997. Neuroanatomical correlates of pleasant and unpleasant emotion. Neuropsychologia 35, 1437-1444. https://doi.org/10.1016/S00283932(97)00070-5

Langers, D.R.M., Melcher, J.R., 2011. Hearing Without Listening: Functional Connectivity Reveals the Engagement of Multiple Nonauditory Networks During Basic Sound Processing. Brain Connectivity 1, 233-244. https://doi.org/10.1089/brain.2011.0023

Laroche, S., Neuenschwander-el Massioui, N., Edeline, J.-M., Dutrieux, G., 1987. Hippocampal associative cellular responses: dissociation with behavioral responses revealed by a transfer-of-control technique. Behavioral and Neural Biology 47, 356-368. https://doi.org/10.1016/S0163-1047(87)90474-2

Laureano, M.R., Onishi, E.T., Bressan, R.A., Castiglioni, M.L.V., Batista, I.R., Reis, M.A., Garcia, M.V., de Andrade, A.N., de Almeida, R.R., Garrido, G.J., Jackowski, A.P., 2014. Memory Networks in Tinnitus: A Functional Brain Image Study. PLoS ONE 9, e87839. https://doi.org/10.1371/journal.pone.0087839

Lavasani, A.N., Mohammadkhani, G., Motamedi, M., Karimi, L.J., Jalaie, S., 2013. Evaluation of temporal resolution in patients with unilateral temporal lobe epilepsy by the gaps-in-noise test. Audiology 22, 75-84.

LeDoux, J.E., Ruggiero, D.A., Reis, Donald.J., 1985. Projections to the subcortical forebrain from anatomically defined regions of the medial geniculate body in the rat. J. Comp. Neurol. 242, 182-213. https://doi.org/10.1002/cne.902420204

Lee, A.C.H., Yeung, L.-K., Barense, M.D., 2012. The hippocampus and visual perception. Front. Hum. Neurosci. 6. https://doi.org/10.3389/fnhum.2012.00091

Lee, I., Kim, J., 2010. The shift from a response strategy to object-in-place strategy during learning is accompanied by a matching shift in neural firing correlates in the hippocampus. Learn. Mem. 17, 381-393. https://doi.org/10.1101/lm.1829110

Lee, S.-M., Kim, B.-K., Kim, T.-W., Ji, E.-S., Choi, H.-H., 2016. Music application alleviates short-term memory impairments through increasing cell proliferation in the hippocampus of valproic acid-induced autistic rat pups. Journal of Exercise Rehabilitation 12, 148-155. https://doi.org/10.12965/jer.1632638.319

Lehmann, H., Sparks, F.T., Spanswick, S.C., Hadikin, C., McDonald, R.J., Sutherland, R.J., 2009. Making context memories independent of the hippocampus. Learning \& Memory 16, 417-420. https://doi.org/10.1101/lm.1385409

Lehne, M., Rohrmeier, M., Koelsch, S., 2014. Tension-related activity in the orbitofrontal cortex and amygdala: an fMRI study with music. Social Cognitive and Affective Neuroscience 9, 1515-1523. https://doi.org/10.1093/scan/nst141

Leitman, 2010. "It's not what you say, but how you say it": a reciprocal temporo-frontal network for affective prosody. Frontiers in Human Neuroscience. https://doi.org/10.3389/fnhum.2010.00019

Lennox, B.R., Park, S.B.G., Medley, I., Morris, P.G., Jones, P.B., 2000. The functional anatomy of auditory hallucinations in schizophrenia. Psychiatry Research: Neuroimaging 100,13-20. https://doi.org/10.1016/S0925-4927(00)00068-8

Lerner, Y., Papo, D., Zhdanov, A., Belozersky, L., Hendler, T., 2009. Eyes Wide Shut: Amygdala Mediates Eyes-Closed Effect on Emotional Experience with Music. PLoS ONE 4, e6230. https://doi.org/10.1371/journal.pone.0006230

Leutgeb, S., Leutgeb, J.K., Treves, A., Moser, M.-B., Moser, E.I., 2004. Distinct Ensemble Codes in Hippocampal Areas CA3 and CA1. Science 305, 1295-1298. https://doi.org/10.1126/science.1100265

Lever, C., Burton, S., Jeewajee, A., O'Keefe, J., Burgess, N., 2009. Boundary Vector Cells in the Subiculum of the Hippocampal Formation. Journal of Neuroscience 29, 9771-9777. https://doi.org/10.1523/JNEUROSCI.1319-09.2009

Li, K., Jia, H., She, X., Cui, B., Zhang, N., Chen, X., Xu, C., An, G., Ma, Q., 2014. Role of NMDA receptors in noise-induced tau hyperphosphorylation in rat hippocampus and prefrontal cortex. Journal of the Neurological Sciences 340, 191-197. https://doi.org/10.1016/j.jns.2014.03.027

Li, S., Ma, L., Wang, Y., Wang, X., Li, Y., Qin, L., 2018. Auditory steady-state responses in primary and non-primary regions of the auditory cortex in neonatal ventral hippocampal lesion rats. PLOS ONE 13, e0192103. https://doi.org/10.1371/journal.pone.0192103

Liang, J.C., Wagner, A.D., Preston, A.R., 2013. Content Representation in the Human Medial Temporal Lobe. Cerebral Cortex 23, 80-96. https://doi.org/10.1093/cercor/bhr379

Liberman, T., Velluti, R.A., Pedemonte, M., 2009. Temporal correlation between auditory neurons and the hippocampal theta rhythm induced by novel stimulations in awake guinea pigs. Brain Research 1298, 70-77. https://doi.org/10.1016/j.brainres.2009.08.061

Liberson, W.T., Cadilhac, J.G., 1953. Electroshock and Rhinencephalic Seizure States. Stereotactic and Functional Neurosurgery 13, 278-286. https://doi.org/10.1159/000105425 
Liddle, P.F., Friston, K.J., Frith, C.D., Hirsch, S.R., Jones, T., Frackowiak, R.S.J., 1992. Patterns of Cerebral Blood Flow in Schizophrenia. British Journal of Psychiatry 160, 179-186. https://doi.org/10.1192/bjp.160.2.179

Lidsky, T.I., Levine, M.S., MacGregor, S., 1974. Tonic and phasic effects evoked concurrently by sensory stimuli in hippocampal units. Experimental Neurology 44, 130-134.

Lisman, J., Buzsáki, G., Eichenbaum, H., Nadel, L., Ranganath, C., Redish, A.D., 2017. Viewpoints: how the hippocampus contributes to memory, navigation and cognition. Nature Neuroscience 20, 1434-1447. https://doi.org/10.1038/nn.4661

Lisman, J.E., Grace, A.A., 2005. The Hippocampal-VTA Loop: Controlling the Entry of Information into Long-Term Memory. Neuron 46, 703-713. https://doi.org/10.1016/j.neuron.2005.05.002

Liu, J., Lin, T., Yan, X., Jiang, W., Shi, M., Ye, R., Rao, Z., Zhao, G., 2010. Effects of infrasound on cell proliferation in the dentate gyrus of adult rats: NeuroReport 21, 585-589. https://doi.org/10.1097/WNR.0b013e32833a7dc4

Livingston, G., Sommerlad, A., Orgeta, V., Costafreda, S.G., Huntley, J., Ames, D., Ballard, C., Banerjee, S., Burns, A., CohenMansfield, J., Cooper, C., Fox, N., Gitlin, L.N., Howard, R., Kales, H.C., Larson, E.B., Ritchie, K., Rockwood, K., Sampson, E.L., Samus, Q., Schneider, L.S., Selbæk, G., Teri, L., Mukadam, N., 2017. Dementia prevention, intervention, and care. The Lancet 390, 2673-2734. https://doi.org/10.1016/S0140-6736(17)31363-6

Lockwood, A.H., Salvi, R.J., Coad, M.L., Towsley, M.L., Wack, D.S., Murphy, B.W., 1998. The functional neuroanatomy of tinnitus. Neurology 50, 114-120.

Loechner, K.J., Weisz, D.J., 1987. Hippocampectomy and feature-positive discrimination. Behavioural Brain Research 26, 63-73. https://doi.org/10.1016/0166-4328(87)90017-9

Logan, C.G., Grafton, S.T., 1995. Functional anatomy of human eyeblink conditioning determined with regional cerebral glucose metabolism and positron-emission tomography. Proceedings of the National Academy of Sciences 92, 7500-7504. https://doi.org/10.1073/pnas.92.16.7500

Long, L.L., Hinman, J.R., Chen, C.-M.A., Stevenson, I.H., Read, H.L., Escabi, M.A., Chrobak, J.J., 2014. Novel acoustic stimuli can alter locomotor speed to hippocampal theta relationship: Sound and Hippocampal Theta. Hippocampus 24, 1053 1058. https://doi.org/10.1002/hipo.22308

Lositsky, O., Chen, J., Toker, D., Honey, C.J., Shvartsman, M., Poppenk, J.L., Hasson, U., Norman, K.A., 2016. Neural pattern change during encoding of a narrative predicts retrospective duration estimates. eLife 5, 40.

Love, S., Pollick, F.E., Latinus, M., 2011. Cerebral Correlates and Statistical Criteria of Cross-Modal Face and Voice Integration. Seeing and Perceiving 24, 351-367. https://doi.org/10.1163/187847511X584452

Mably, A.J., Colgin, L.L., 2018. Gamma oscillations in cognitive disorders. Current Opinion in Neurobiology 52, $182-187$. https://doi.org/10.1016/j.conb.2018.07.009

MacDonald, C.J., Carrow, S., Place, R., Eichenbaum, H., 2013. Distinct Hippocampal Time Cell Sequences Represent Odor Memories in Immobilized Rats. Journal of Neuroscience 33, 14607-14616. https://doi.org/10.1523/JNEUROSCI.1537-13.2013

MacDonald, C.J., Lepage, K.Q., Eden, U.T., Eichenbaum, H., 2011. Hippocampal “Time Cells” Bridge the Gap in Memory for Discontiguous Events. Neuron 71, 737-749. https://doi.org/10.1016/j.neuron.2011.07.012

Macedo, C.E., Angst, M.-J., Guiberteau, T., Brasse, D., O’Brien, T.J., Sandner, G., 2010. Acoustic hypersensitivity in adult rats after neonatal ventral hippocampus lesions. Behavioural Brain Research 207, 161-168. https://doi.org/10.1016/j.bbr.2009.10.002

Maguire, E.A., Gadian, D.G., Johnsrude, I.S., Good, C.D., Ashburner, J., Frackowiak, R.S.J., Frith, C.D., 2000. Navigationrelated structural change in the hippocampi of taxi drivers. Proceedings of the National Academy of Sciences 97, 4398-4403. https://doi.org/10.1073/pnas.070039597

Maguire, E.A., Mullally, S.L., 2013. The hippocampus: A manifesto for change. Journal of Experimental Psychology: General 142, 1180-1189. https://doi.org/10.1037/a0033650

Maller, J.J., Daskalakis, Z.J., Thomson, R.H.S., Daigle, M., Barr, M.S., Fitzgerald, P.B., 2012. Hippocampal volumetrics in treatment-resistant depression and schizophrenia: The devil's in De-Tail. Hippocampus 22, 9-16. https://doi.org/10.1002/hipo.20873

Maller, J.J., Welton, T., Middione, M., Callaghan, F.M., Rosenfeld, J.V., Grieve, S.M., 2019. Revealing the Hippocampal Connectome through Super-Resolution 1150-Direction Diffusion MRI. Scientific Reports 9. https://doi.org/10.1038/s41598-018-37905-9

Manan, H.A., Franz, E.A., Yusoff, A.N., Sarah Mukari, S.Z.-M., 2012. Hippocampal-cerebellar involvement in enhancement of performance in word-based BRT with the presence of background noise: An initial fMRI study. Psychology \& Neuroscience 5, 247-256. https://doi.org/10.3922/j.psns.2012.2.16

Manikandan, S., Padma, M.K., Srikumar, R., Jeya Parthasarathy, N., Muthuvel, A., Devi, R.S., 2006. Effects of chronic noise stress on spatial memory of rats in relation to neuronal dendritic alteration and free radical-imbalance in hippocampus and medial prefrontal cortex. Neuroscience Letters 399, 17-22. https://doi.org/10.1016/j.neulet.2006.01.037

Manns, J.R., Howard, M.W., Eichenbaum, H., 2007. Gradual Changes in Hippocampal Activity Support Remembering the Order of Events. Neuron 56, 530-540. https://doi.org/10.1016/j.neuron.2007.08.017

Manukyan, A.L., 2022. Noise as a cause of neurodegenerative disorders: molecular and cellular mechanisms. Neurol Sci. https://doi.org/10.1007/s10072-022-05948-6

Marr, D., 1971. Simple memory: a theory for archicortex. Philosophical Transactions of the Royal Society of London. Series B: Biological Sciences 262, 23-81.

Martins, M.J.D., Fischmeister, F.Ph.S., Gingras, B., Bianco, R., Puig-Waldmueller, E., Villringer, A., Fitch, W.T., Beisteiner, R., 2020. Recursive music elucidates neural mechanisms supporting the generation and detection of melodic hierarchies. Brain Struct Funct 225, 1997-2015. https://doi.org/10.1007/s00429-020-02105-7

Martorell, A.J., Paulson, A.L., Suk, H.-J., Abdurrob, F., Drummond, G.T., Guan, W., Young, J.Z., Kim, D.N.-W., Kritskiy, O., Barker, S.J., Mangena, V., Prince, S.M., Brown, E.N., Chung, K., Boyden, E.S., Singer, A.C., Tsai, L.-H., 2019. Multisensory Gamma Stimulation Ameliorates Alzheimer's-Associated Pathology and Improves Cognition. Cell 177, 256- 
271.e22. https://doi.org/10.1016/j.cell.2019.02.014

Matsubara, T., Ogata, K., Hironaga, N., Kikuchi, Y., Uehara, T., Chatani, H., Mitsudo, T., Shigeto, H., Tobimatsu, S., 2018.

Altered neural synchronization to pure tone stimulation in patients with mesial temporal lobe epilepsy: An MEG study. Epilepsy \& Behavior 88, 96-105. https://doi.org/10.1016/j.yebeh.2018.08.036

Matt, L., Eckert, P., Panford-Walsh, R., Geisler, H.-S., Bausch, A.E., Manthey, M., Müller, N.I.C., Harasztosi, C., Rohbock, K., Ruth, P., Friauf, E., Ott, T., Zimmermann, U., Rüttiger, L., Schimmang, T., Knipper, M., Singer, W., 2018. Visualizing BDNF Transcript Usage During Sound-Induced Memory Linked Plasticity. Frontiers in Molecular Neuroscience 11. https://doi.org/10.3389/fnmol.2018.00260

Mau, W., Sullivan, D.W., Kinsky, N.R., Hasselmo, M.E., Howard, M.W., Eichenbaum, H., 2018. The Same Hippocampal CA1 Population Simultaneously Codes Temporal Information over Multiple Timescales. Current Biology 28, 1499-1508.e4. https://doi.org/10.1016/j.cub.2018.03.051

Mayes, A.R., Holdstock, J.S., Isaac, C.L., Montaldi, D., Grigor, J., Gummer, A., Cariga, P., Downes, J.J., Tsivilis, D., Gaffan, D., Gong, Q., Norman, K.A., 2004. Associative recognition in a patient with selective hippocampal lesions and relatively normal item recognition. Hippocampus 14, 763-784. https://doi.org/10.1002/hipo.10211

Mays, L.E., Best, P.J., 1975. Hippocampal unit activity to tonal stimuli during arousal from sleep and in awake rats. Experimental Neurology 47, 268-279. https://doi.org/10.1016/0014-4886(75)90256-3

McCarthy, G., Wood, C., Williamson, P., Spencer, D., 1989. Task-dependent field potentials in human hippocampal formation. The Journal of Neuroscience 9, 4253-4268. https://doi.org/10.1523/JNEUROSCI.09-12-04253.1989

McClelland, J.L., O’Reilly, R.C., 1995. Why There Are Complementary Learning Systems in the Hippocampus and Neocortex:InsightsFrom the Successesand Failuresof Connectionist Models of Learning and Memory. Psychological Review 102, 419-457.

McCormick, C., Dalton, M.A., Zeidman, P., Maguire, E.A., 2021. Characterising the hippocampal response to perception, construction and complexity. Cortex 137, 1-17. https://doi.org/10.1016/j.cortex.2020.12.018

McCormick, D.A., Clark, G.A., Lavond, D.G., Thompson, R.F., 1982. Initial localization of the memory trace for a basic form of learning. Proceedings of the National Academy of Sciences 79, 2731-2735. https://doi.org/10.1073/pnas.79.8.2731

McEchron, M.D., Disterhoft, J.F., 1997. Sequence of Single Neuron Changes in CA1 Hippocampus of Rabbits During Acquisition of Trace Eyeblink Conditioned Responses. Journal of Neurophysiology 78, 1030-1044. https://doi.org/10.1152/jn.1997.78.2.1030

McEchron, M.D., Tseng, W., Disterhoft, J.F., 2003. Single Neurons in CA1 Hippocampus Encode Trace Interval Duration during Trace Heart Rate (Fear) Conditioning in Rabbit. The Journal of Neuroscience 23, 1535-1547. https://doi.org/10.1523/JNEUROSCI.23-04-01535.2003

McGuire, P.K., Murray, R.M., Shah, G.M.S., 1993. Increased blood flow in Broca's area during auditory hallucinations in schizophrenia. The Lancet 342, 703-706. https://doi.org/10.1016/0140-6736(93)91707-S

McKenzie, S., Frank, A.J., Kinsky, N.R., Porter, B., Rivière, P.D., Eichenbaum, H., 2014. Hippocampal Representation of Related and Opposing Memories Develop within Distinct, Hierarchically Organized Neural Schemas. Neuron 83, 202 215. https://doi.org/10.1016/j.neuron.2014.05.019

McMillan, T.M., Powell, G.E., Janota, I., Polkey, C.E., 1987. Relationships between neuropathology and cognitive functioning in temporal lobectomy patients. Journal of Neurology, Neurosurgery \& Psychiatry 50, 167-176. https://doi.org/10.1136/jnnp.50.2.167

McNaughton, B.L., Morris, R.G.M., 1987. Hippocampal synaptic enhancement and information storage within a distributed memory system. Trends in Neurosciences 10, 408-415.

Meck, W.H., Church, R.M., Olton, D.S., 1984. Hippocampus, time, and memory. Behavioral Neuroscience 98, 3-22.

Medoff, D.R., Holcomb, H.H., Lahti, A.C., Tamminga, C.A., 2001. Probing the human hippocampus using rCBF: Contrasts in schizophrenia. Hippocampus 11, 543-550. https://doi.org/10.1002/hipo.1070

Mehta, M.R., Quirk, M.C., Wilson, M.A., 2000. Experience-Dependent Asymmetric Shape of Hippocampal Receptive Fields. Neuron 25, 707-715. https://doi.org/10.1016/S0896-6273(00)81072-7

Meister, M.L.R., Buffalo, E.A., 2018. Neurons in Primate Entorhinal Cortex Represent Gaze Position in Multiple Spatial Reference Frames. J. Neurosci. 38, 2430-2441. https://doi.org/10.1523/JNEUROSCI.2432-17.2018

Melcher, J.R., Knudson, I.M., Levine, R.A., 2013. Subcallosal brain structure: Correlation with hearing threshold at supra-clinical frequencies $(>8 \mathrm{kHz})$, but not with tinnitus. Hearing Research 295, 79-86. https://doi.org/10.1016/j.heares.2012.03.013

Meng, B., Zhu, S., Li, S., Zeng, Q., Mei, B., 2009. Global view of the mechanisms of improved learning and memory capability in mice with music-exposure by microarray. Brain Research Bulletin 80, 36-44. https://doi.org/10.1016/j.brainresbull.2009.05.020

Meyer, S.S., Bonaiuto, J., Lim, M., Rossiter, H., Waters, S., Bradbury, D., Bestmann, S., Brookes, M., Callaghan, M.F., Weiskopf, N., Barnes, G.R., 2017. Flexible head-casts for high spatial precision MEG. Journal of Neuroscience Methods 276, 38 45. https://doi.org/10.1016/j.jneumeth.2016.11.009

Meyer, V., Yates, A.J., 1955. Intellectual changes following temporal lobectomy for psychomotor epilepsy: Preliminary communication. Journal of Neurology, Neurosurgery \& Psychiatry 18, 44-52. https://doi.org/10.1136/jnnp.18.1.44

Micco, D.J., Schwartz, M., 1971. Effects of hippocampal lesions upon the development of Pavlovian internal inhibition in rats. Journal of Comparative and Physiological Psychology 76, 371-377. https://doi.org/10.1037/h0031376

Michelmann, S., Price, A.R., Aubrey, B., Strauss, C.K., Doyle, W.K., Friedman, D., Dugan, P.C., Devinsky, O., Devore, S., Flinker, A., Hasson, U., Norman, K.A., 2021. Moment-by-moment tracking of naturalistic learning and its underlying hippocampo-cortical interactions. Nat Commun 12, 5394. https://doi.org/10.1038/s41467-021-25376-y

Miller, C.L., Freedman, R., 1995. The activity of hippocampal interneurons and pyramidal cells during the response of the hippocampus to repeated auditory stimuli. Neuroscience 69, 371-381. https://doi.org/10.1016/0306-4522(95)00249-I

Miller, C.L., Freedman, R., 1993. Medial septal neuron activity in relation to an auditory sensory gating paradigm. Neuroscience 55, 373-380. https://doi.org/10.1016/0306-4522(93)90506-B 
Milner, B., 1972. Disorders of Learning and Memory after Temporal Lobe Lesions in Man. Neurosurgery 19, 421-446. https://doi.org/10.1093/neurosurgery/19.CN_suppl_1.421

Milner, B., 1962. Les troubles de la mémoire accompagnant des lésions hippocampiques bilatérales, in: Passouant, P. (Ed.), Physiologie de l'Hippocampe. Centre National de la Recherche Scientifique, Paris, pp. 257-272.

Milner, B., Teuber, H.L., 1968. Alteration of perception and memory in man: Reflections on methods, in: Weiskrantz, L. (Ed.), Analysis of Behavioral Change. Harper \& Row, New York.

Mirz, F., 2000. Cortical Networks Subserving the Perception of Tinnitus - a PET Study. Acta Oto-Laryngologica 120, $241-243$. https://doi.org/10.1080/000164800454503

Mirz, F., Gjedde, A., Sdkilde-Jrgensen, H., Pedersen, C.B., 2000. Functional brain imaging of tinnitus-like perception induced by aversive auditory stimuli: NeuroReport 11, 633-637. https://doi.org/10.1097/00001756-200002280-00039

Mitchell, R.L.C., Elliott, R., Barry, M., Cruttenden, A., Woodruff, P.W.R., 2003. The neural response to emotional prosody, as revealed by functional magnetic resonance imaging. Neuropsychologia 41, 1410-1421. https://doi.org/10.1016/S0028-3932(03)00017-4

Modi, M.N., Dhawale, A.K., Bhalla, U.S., 2014. CA1 cell activity sequences emerge after reorganization of network correlation structure during associative learning. eLife 3. https://doi.org/10.7554/eLife.01982

Moita, M.A.P., Rosis, S., Zhou, Y., LeDoux, J.E., Blair, H.T., 2004. Putting Fear in Its Place: Remapping of Hippocampal Place Cells during Fear Conditioning. Journal of Neuroscience 24, 7015-7023. https://doi.org/10.1523/JNEUROSCI.549203.2004

Moita, M.A.P., Rosis, S., Zhou, Y., LeDoux, J.E., Blair, H.T., 2003. Hippocampal Place Cells Acquire Location-Specific Responses to the Conditioned Stimulus during Auditory Fear Conditioning. Neuron 37, 485-497. https://doi.org/10.1016/S0896-6273(03)00033-3

Momennejad, I., 2020. Learning Structures: Predictive Representations, Replay, and Generalization. Current Opinion in Behavioral Sciences 32, 155-166. https://doi.org/10.1016/j.cobeha.2020.02.017

Mount, R.A., Sridhar, S., Hansen, K.R., Mohammed, A.I., Abdulkerim, M., Kessel, R., Nazer, B., Gritton, H.J., Han, X., 2021. Distinct neuronal populations contribute to trace conditioning and extinction learning in the hippocampal CA1. eLife 10, e56491. https://doi.org/10.7554/eLife.56491

Moxon, K.A., Gerhardt, G.A., Bickford, P.C., Austin, K., Rose, G.M., Woodward, D.J., Adler, L.E., 1999. Multiple single units and population responses during inhibitory gating of hippocampal auditory response in freely-moving rats. Brain Research 825, 75-85. https://doi.org/10.1016/S0006-8993(99)01187-7

Mueller, K., Fritz, T., Mildner, T., Richter, M., Schulze, K., Lepsien, J., Schroeter, M.L., Möller, H.E., 2015. Investigating the dynamics of the brain response to music: A central role of the ventral striatum/nucleus accumbens. NeuroImage 116, 68-79. https://doi.org/10.1016/j.neuroimage.2015.05.006

Mueller, K., Mildner, T., Fritz, T., Lepsien, J., Schwarzbauer, C., Schroeter, M.L., Möller, H.E., 2011. Investigating brain response to music: A comparison of different fMRI acquisition schemes. NeuroImage 54, 337-343. https://doi.org/10.1016/j.neuroimage.2010.08.029

Mueller, S.G., Laxer, K.D., Scanlon, C., Garcia, P., McMullen, W.J., Loring, D.W., Meador, K.J., Weiner, M.W., 2012. Different structural correlates for verbal memory impairment in temporal lobe epilepsy with and without mesial temporal lobe sclerosis. Human Brain Mapping 33, 489-499. https://doi.org/10.1002/hbm.21226

Mullally, S.L., Intraub, H., Maguire, E.A., 2012. Attenuated Boundary Extension Produces a Paradoxical Memory Advantage in Amnesic Patients. Current Biology 22, 261-268. https://doi.org/10.1016/j.cub.2012.01.001

Müller, N., Keil, J., Obleser, J., Schulz, H., Grunwald, T., Bernays, R.-L., Huppertz, H.-J., Weisz, N., 2013. You can’t stop the music: Reduced auditory alpha power and coupling between auditory and memory regions facilitate the illusory perception of music during noise. NeuroImage 79, 383-393. https://doi.org/10.1016/i.neuroimage.2013.05.001

Muller, R., Kubie, J., 1987. The effects of changes in the environment on the spatial firing of hippocampal complex-spike cells. J. Neurosci. 7, 1951-1968. https://doi.org/10.1523/JNEUROSCI.07-07-01951.1987

Muñoz, M., Insausti, R., 2005. Cortical efferents of the entorhinal cortex and the adjacent parahippocampal region in the monkey (Macaca fascicularis). European Journal of Neuroscience 22, 1368-1388. https://doi.org/10.1111/j.14609568.2005.04299.x

Munoz-Lopez, M., Insausti, R., Mohedano-Moriano, A., Mishkin, M., Saunders, R.C., 2015. Anatomical pathways for auditory memory II: Information from rostral superior temporal gyrus to dorsolateral temporal pole and medial temporal cortex. Frontiers in Neuroscience 9.

Munoz-Lopez, M.M., Mohedano-Moriano, A., Insausti, R., 2010. Anatomical Pathways for Auditory Memory in Primates. Frontiers in Neuroanatomy 4. https://doi.org/10.3389/fnana.2010.00129

Musalek, M., Podreka, I., Walter, H., Suess, E., Passweg, V., Nutzinger, D., Strobl, R., Lesch, O.M., 1989. Regional brain function in hallucinations: A study of regional cerebral blood flow with 99m-Tc-HMPAO-SPECT in patients with auditory hallucinations, tactile hallucinations, and normal controls. Comprehensive Psychiatry 30, 99-108. https://doi.org/10.1016/0010-440X(89)90123-5

Mutschler, I., Wieckhorst, B., Speck, O., Schulze-Bonhage, A., Hennig, J., Seifritz, E., Ball, T., 2010. Time Scales of Auditory Habituation in the Amygdala and Cerebral Cortex. Cerebral Cortex 20, 2531-2539. https://doi.org/10.1093/cercor/bhq001

Muzzio, I.A., Levita, L., Kulkarni, J., Monaco, J., Kentros, C., Stead, M., Abbott, L.F., Kandel, E.R., 2009. Attention Enhances the Retrieval and Stability of Visuospatial and Olfactory Representations in the Dorsal Hippocampus. PLoS Biol 7 , e1000140. https://doi.org/10.1371/journal.pbio.1000140

Näätänen, R., 1990. The role of attention in auditory information processing as revealed by event-related potentials and other brain measures of cognitive function. Behav Brain Sci 13, 201-233. https://doi.org/10.1017/S0140525X00078407

Nadel, L., Samsonovich, A., Ryan, L., Moscovitch, M., 2000. Multiple trace theory of human memory: Computational, neuroimaging, and neuropsychological results. Hippocampus 10, 352-368. https://doi.org/10.1002/10981063(2000)10:4<352::AID-HIPO2>3.0.CO;2-D 
Nadhimi, Y., Llano, D.A., 2021. Does hearing loss lead to dementia? A review of the literature. Hearing Research 402, 108038. https://doi.org/10.1016/j.heares.2020.108038

Nani, A., Manuello, J., Liloia, D., Duca, S., Costa, T., Cauda, F., 2019. The Neural Correlates of Time: A Meta-analysis of Neuroimaging Studies. Journal of Cognitive Neuroscience 31, 1796-1826. https://doi.org/10.1162/jocn_a_01459

Nau, M., Navarro Schröder, T., Bellmund, J.L.S., Doeller, C.F., 2018. Hexadirectional coding of visual space in human entorhinal cortex. Nature Neuroscience 21, 188-190. https://doi.org/10.1038/s41593-017-0050-8

Ngo, H.-V.V., Martinetz, T., Born, J., Mölle, M., 2013. Auditory Closed-Loop Stimulation of the Sleep Slow Oscillation Enhances Memory. Neuron 78, 545-553. https://doi.org/10.1016/j.neuron.2013.03.006

Niki, H., 1967. Effects of Hippocampal Ablation on Learning in the Rat, in: Progress in Brain Research. Elsevier, pp. 305-317. https://doi.org/10.1016/S0079-6123(08)63107-6

Nilssen, E.S., Doan, T.P., Nigro, M.J., Ohara, S., Witter, M.P., 2019. Neurons and networks in the entorhinal cortex: A reappraisal of the lateral and medial entorhinal subdivisions mediating parallel cortical pathways. Hippocampus. https://doi.org/10.1002/hipo.23145

Nir, Y., Staba, R.J., Andrillon, T., Vyazovskiy, V.V., Cirelli, C., Fried, I., Tononi, G., 2011. Regional Slow Waves and Spindles in Human Sleep. Neuron 70, 153-169. https://doi.org/10.1016/j.neuron.2011.02.043

Noreña, A.J., 2011. An integrative model of tinnitus based on a central gain controlling neural sensitivity. Neuroscience \& Biobehavioral Reviews 35, 1089-1109. https://doi.org/10.1016/j.neubiorev.2010.11.003

Norman, Y., Yeagle, E.M., Khuvis, S., Harel, M., Mehta, A.D., Malach, R., 2019. Hippocampal sharp-wave ripples linked to visual episodic recollection in humans. Science 365, eaax1030. https://doi.org/10.1126/science.aax1030

Nourski, K.V., Reale, R.A., Oya, H., Kawasaki, H., Kovach, C.K., Chen, H., Howard, M.A., Brugge, J.F., 2009. Temporal Envelope of Time-Compressed Speech Represented in the Human Auditory Cortex. Journal of Neuroscience 29, 15564-15574. https://doi.org/10.1523/JNEUROSCI.3065-09.2009

O’Brien, C.E., Bowden, S.C., Bardenhagen, F.J., Cook, M.J., 2003. Neuropsychological correlates of hippocampal and rhinal cortex volumes in patients with mesial temporal sclerosis. Hippocampus 13, 892-904. https://doi.org/10.1002/hipo.10128

O'Connor, J.J., Rowan, M.J., Anwyl, R., 1992. Serotoninergic depression of auditory evoked responses recorded in the rat hippocampus: effect of repeated buspirone treatment. Brain Research 573, 190-196. https://doi.org/10.1016/00068993(92) $90762-\mathrm{X}$

O'Keefe, J., Burgess, N., 1996. Geometric determinants of the place fields of hippocampal neurons. Nature 381, 425-428. https://doi.org/10.1038/381425a0

O'Keefe, J., Dostrovsky, J., 1971. The hippocampus as a spatial map. Preliminary evidence from unit activity in the freely-moving rat. Brain Research 34, 171-175. https://doi.org/10.1016/0006-8993(71)90358-1

O’Keefe, J., Krupic, J., 2021. Do hippocampal pyramidal cells respond to nonspatial stimuli? Physiological Reviews 101, 14271456. https://doi.org/10.1152/physrev.00014.2020

O’Keefe, J., Recce, M.L., 1993. Phase relationship between hippocampal place units and the EEG theta rhythm. Hippocampus 3, 317-330. https://doi.org/10.1002/hipo.450030307

Olds, J., Hirano, T., 1969. Conditioned responses of hippocampal and other neurons. Electroencephalography and Clinical Neurophysiology 26, 159-166. https://doi.org/10.1016/0013-4694(69)90206-5

Olsen, R.K., Moses, S.N., Riggs, L., Ryan, J.D., 2012. The hippocampus supports multiple cognitive processes through relational binding and comparison. Front. Hum. Neurosci. 6. https://doi.org/10.3389/fnhum.2012.00146

Omar, R., Henley, S.M.D., Bartlett, J.W., Hailstone, J.C., Gordon, E., Sauter, D.A., Frost, C., Scott, S.K., Warren, J.D., 2011. The structural neuroanatomy of music emotion recognition: Evidence from frontotemporal lobar degeneration. NeuroImage 56, 1814-1821. https://doi.org/10.1016/i.neuroimage.2011.03.002

Ong, J.L., Patanaik, A., Chee, N.I.Y.N., Lee, X.K., Poh, J.-H., Chee, M.W.L., 2018. Auditory stimulation of sleep slow oscillations modulates subsequent memory encoding through altered hippocampal function. Sleep 41. https://doi.org/10.1093/sleep/zsy031

Onoda, K., Takahashi, E., Sakata, S., 2003. Event-related potentials in the frontal cortex, hippocampus, and cerebellum during a temporal discrimination task in rats. Cognitive Brain Research 17, 380-387. https://doi.org/10.1016/S09266410(03)00139-3

O’Reilly, R.C., McClelland, J.L., 1994. Hippocampal conjunctive encoding, storage, and recall: Avoiding a trade-off. Hippocampus 4, 661-682. https://doi.org/10.1002/hipo.450040605

Orsmond, G.I., Miller, L.K., 1999. Cognitive, Musical and Environmental Correlates of Early Music Instruction. Psychology of Music 27, 18-37. https://doi.org/10.1177/0305735699271003

O'Sullivan, A.E., Lim, C.Y., Lalor, E.C., 2019. Look at me when I'm talking to you: Selective attention at a multisensory cocktail party can be decoded using stimulus reconstruction and alpha power modulations. European Journal of Neuroscience. https://doi.org/10.1111/ejn.14425

Pallesen, K.J., Brattico, E., Bailey, C.J., Korvenoja, A., Gjedde, A., 2009. Cognitive and Emotional Modulation of Brain Default Operation. Journal of Cognitive Neuroscience 21, 1065-1080. https://doi.org/10.1162/jocn.2009.21086

Papez, J.W., 1937. A proposed mechanism of emotion. Archives of Neurology And Psychiatry 38, 725-743. https://doi.org/10.1001/archneurpsyc.1937.02260220069003

Papp, G., Kovac, S., Frese, A., Evers, S., 2014. The impact of temporal lobe epilepsy on musical ability. Seizure 23, 533-536. https://doi.org/10.1016/j.seizure.2014.03.018

Paquette, S., Fournier, P., Dupont, S., Szabo de Edelenyi, F., Galan, P., Samson, S., 2017. Risk of Tinnitus After Medial Temporal Lobe Surgery. JAMA Neurology 74, 1376. https://doi.org/10.1001/jamaneurol.2017.2718

Park, H., Rugg, M.D., 2009. Prestimulus hippocampal activity predicts later recollection. Hippocampus NA-NA. https://doi.org/10.1002/hipo.20663

Parmeggiani, P.L., Rapisarda, C., 1969. Hippocampal output and sensory mechanisms. Brain Research 14, 387-400. https://doi.org/10.1016/0006-8993(69)90117-6 
Pastalkova, E., Itskov, V., Amarasingham, A., Buzsaki, G., 2008. Internally Generated Cell Assembly Sequences in the Rat Hippocampus. Science 321, 1322-1327. https://doi.org/10.1126/science.1159775

Patterson, M., Olah, J., Clement, J., 1977. Classical nictitating membrane conditioning in the awake, normal, restrained cat. Science 196, 1124-1126. https://doi.org/10.1126/science.870974

Paz, R., Gelbard-Sagiv, H., Mukamel, R., Harel, M., Malach, R., Fried, I., 2010. A neural substrate in the human hippocampus for linking successive events. Proceedings of the National Academy of Sciences 107, 6046-6051. https://doi.org/10.1073/pnas.0910834107

Pedemonte, M., Pérez-Perera, L., Peña, J.L., Velluti, R.A., 2001. Sleep and Wakefulness Auditory Processing: Cortical Units vs. Hippocampal Theta Rhythm. Sleep Research Online 4, 51-57.

Pedemonte, M., Pe a, J., Velluti, RicardoA., 1996. Firing of inferior colliculus auditory neurons is phase-locked to the hippocampus theta rhythm during paradoxical sleep and waking. Experimental Brain Research 112. https://doi.org/10.1007/BF00227176

Penfield, W., Milner, B., 1958. Memory Deficit Produced by Bilateral Lesions in the Hippocampal Zone 23.

Pereira, C.S., Teixeira, J., Figueiredo, P., Xavier, J., Castro, S.L., Brattico, E., 2011. Music and Emotions in the Brain: Familiarity Matters. PLoS ONE 6, e27241. https://doi.org/10.1371/journal.pone.0027241

Persson, J., Kalpouzos, G., Nilsson, L.-G., Ryberg, M., Nyberg, L., 2011. Preserved hippocampus activation in normal aging as revealed by fMRI. Hippocampus 21, 753-766. https://doi.org/10.1002/hipo.20794

Pertzov, Y., Miller, T.D., Gorgoraptis, N., Caine, D., Schott, J.M., Butler, C., Husain, M., 2013. Binding deficits in memory following medial temporal lobe damage in patients with voltage-gated potassium channel complex antibody-associated limbic encephalitis. Brain 136, 2474-2485. https://doi.org/10.1093/brain/awt129

Petersson, K.M., Reis, A., Castro-Caldas, A., Ingvar, M., 1999. Effective Auditory-Verbal Encoding Activates the Left Prefrontal and the Medial Temporal Lobes: A Generalization to Illiterate Subjects. NeuroImage 10, 45-54. https://doi.org/10.1006/nimg.1999.0446

Pfeiffer, B.E., Foster, D.J., 2013. Hippocampal place-cell sequences depict future paths to remembered goals. Nature 497, 74-79. https://doi.org/10.1038/nature12112

Phillips, G., LeDoux, E., 1994. Lesions of the Dorsal Hippocampal Formation Interfere with Background but Not Foreground Contextual Fear Conditioning. Learning \& Memory 1, 34-44.

Phillips, M.L., Young, A.W., Scott, S.K., Calder, A.J., Andrew, C., Giampietro, V., Williams, S.C.R., Bullmore, E.T., Brammer, M., Gray, J.A., 1998. Neural responses to facial and vocal expressions of fear and disgust. Proceedings of the Royal Society of London. Series B: Biological Sciences 265, 1809-1817. https://doi.org/10.1098/rspb.1998.0506

Piai, V., Anderson, K.L., Lin, J.J., Dewar, C., Parvizi, J., Dronkers, N.F., Knight, R.T., 2016. Direct brain recordings reveal hippocampal rhythm underpinnings of language processing. Proc Natl Acad Sci USA 113, 11366-11371. https://doi.org/10.1073/pnas.1603312113

Picton, T.W., Hillyard, S.A., Galambos, R., 1976. Habituation and Attention in the Auditory System, in: Keidel, W.D., Neff, W.D. (Eds.), Auditory System, Handbook of Sensory Physiology. Springer Berlin Heidelberg, Berlin, Heidelberg, pp. 343-389. https://doi.org/10.1007/978-3-642-66082-5_8

Pietschnig, J., Voracek, M., Formann, A.K., 2010. Mozart effect-Shmozart effect: A meta-analysis. Intelligence 38, $314-323$. https://doi.org/10.1016/j.intell.2010.03.001

Pizzo, F., Roehri, N., Medina Villalon, S., Trébuchon, A., Chen, S., Lagarde, S., Carron, R., Gavaret, M., Giusiano, B., McGonigal, A., Bartolomei, F., Badier, J.M., Bénar, C.G., 2019. Deep brain activities can be detected with magnetoencephalography. Nat Commun 10, 971. https://doi.org/10.1038/s41467-019-08665-5

Plailly, J., Tillmann, B., Royet, J.-P., 2007. The Feeling of Familiarity of Music and Odors: The Same Neural Signature? Cerebral Cortex 17, 2650-2658. https://doi.org/10.1093/cercor/bhl173

Prabhakar, J., Johnson, E.G., Nordahl, C.W., Ghetti, S., 2018. Memory-related hippocampal activation in the sleeping toddler. Proceedings of the National Academy of Sciences 115, 6500-6505. https://doi.org/10.1073/pnas.1805572115

Qasim, S.E., Fried, I., Jacobs, J., 2021. Phase precession in the human hippocampus and entorhinal cortex. Cell 184, 32423255.e10. https://doi.org/10.1016/j.cell.2021.04.017

Quiroga, R.Q., 2020. No Pattern Separation in the Human Hippocampus. Trends in Cognitive Sciences 24, 994-1007. https://doi.org/10.1016/j.tics.2020.09.012

Quiroga, R.Q., Kraskov, A., Koch, C., Fried, I., 2009. Explicit Encoding of Multimodal Percepts by Single Neurons in the Human Brain. Current Biology 19, 1308-1313. https://doi.org/10.1016/j.cub.2009.06.060

Rabelo, C., Weihing, J., Schochat, E., 2015. Temporal resolution in individuals with neurological disorders. Clinics 70, 606-611. https://doi.org/10.6061/clinics/2015(09)02

Raij, T.T., Valkonen-Korhonen, M., Holi, M., Therman, S., Lehtonen, J., Hari, R., 2009. Reality of auditory verbal hallucinations. Brain 132, 2994-3001. https://doi.org/10.1093/brain/awp186

Ranck, J.B., 1973. Studies on single neurons in dorsal hippocampal formation and septum in unrestrained rats. Experimental Neurology 41, 461-531.

Rausch, R., Crandall, P.H., 1982. Psychological Status Related to Surgical Control of Temporal Lobe Seizures. Epilepsia 23, $191-$ 202. https://doi.org/10.1111/j.1528-1157.1982.tb05067.x

Rauschecker, J.P., Leaver, A.M., Mühlau, M., 2010. Tuning Out the Noise: Limbic-Auditory Interactions in Tinnitus. Neuron 66, 819-826. https://doi.org/10.1016/j.neuron.2010.04.032

Rauschecker, J.P., Scott, S.K., 2016. Pathways and Streams in the Auditory Cortex, in: Neurobiology of Language. Elsevier, pp. 287-298. https://doi.org/10.1016/B978-0-12-407794-2.00024-9

Rauschecker, J.P., Tian, B., 2000. Mechanisms and streams for processing of "what" and "where" in auditory cortex. Proceedings of the National Academy of Sciences 97, 11800-11806. https://doi.org/10.1073/pnas.97.22.11800

Rauscher, F.H., Shaw, G.L., Ky, K.N., 1993. Music and spatial task performance. Nature 365, 1.

Recasens, M., Gross, J., Uhlhaas, P.J., 2018. Low-Frequency Oscillatory Correlates of Auditory Predictive Processing in CorticalSubcortical Networks: A MEG-Study. Scientific Reports 8. https://doi.org/10.1038/s41598-018-32385-3 
Redding, F.K., 1967. Modification of sensory cortical evoked potentials by hippocampal stimulation. Electroencephalography and Clinical Neurophysiology 22, 74-83. https://doi.org/10.1016/0013-4694(67)90009-0

Reddy, L., Zoefel, B., Possel, J.K., Peters, J., Dijksterhuis, D.E., Poncet, M., van Straaten, E.C.W., Baayen, J.C., Idema, S., Self, M.W., 2021. Human Hippocampal Neurons Track Moments in a Sequence of Events. J. Neurosci. 41, 6714-6725. https://doi.org/10.1523/JNEUROSCI.3157-20.2021

Ressler, R.L., Goode, T.D., Kim, S., Ramanathan, K.R., Maren, S., 2021. Covert capture and attenuation of a hippocampusdependent fear memory. Nat Neurosci 24, 677-684. https://doi.org/10.1038/s41593-021-00825-5

Rey, C., Reinecke, H., Besser, R., 1984. Methyltin intoxication in six men; toxicologic and clinical aspects. Vet Hum Toxicol 26, 121-122.

Rickert, E.J., Bennett, T.L., Lane, P., French, J., 1978. Hippocampectomy and the attenuation of blocking. Behavioral Biology 22, 147-160. https://doi.org/10.1016/S0091-6773(78)92170-3

Rickert, E.J., Lorden, J.F., Dawson, R., Smyly, E., Callahan, M.F., 1979. Stimulus processing and stimulus selection in rats with hippocampal lesions. Behavioral and Neural Biology 27, 454-465. https://doi.org/10.1016/S0163-1047(79)92040-5

Rizzolo, L., Leger, M., Corvaisier, S., Groussard, M., Platel, H., Bouet, V., Schumann-Bard, P., Freret, T., 2021. Long-Term Music Exposure Prevents Age-Related Cognitive Deficits in Rats Independently of Hippocampal Neurogenesis. Cerebral Cortex 31, 620-634. https://doi.org/10.1093/cercor/bhaa247

Roberts, B.M., Clarke, A., Addante, R.J., Ranganath, C., 2018. Entrainment enhances theta oscillations and improves episodic memory. Cognitive Neuroscience 9, 181-193. https://doi.org/10.1080/17588928.2018.1521386

Rocchi, F., Oya, H., Balezeau, F., Billig, A.J., Kocsis, Z., Jenison, R., Nourski, K.V., Kovach, C.K., Steinschneider, M., Kikuchi, Y., Rhone, A.E., Dlouhy, B.J., Kawasaki, H., Adolphs, R., Greenlee, J.D.W., Griffiths, T.D., Howard, M.A., Petkov, C.I., 2021. Common Fronto-temporal Effective Connectivity in Humans and Monkeys. Neuron 109, 1-17. https://doi.org/10.1101/2020.04.03.024042

Rolls, E.T., 2013. The mechanisms for pattern completion and pattern separation in the hippocampus. Frontiers in Systems Neuroscience 7. https://doi.org/10.3389/fnsys.2013.00074

Rolls, E.T., 1996. A theory of hippocampal function in memory. Hippocampus 6, 601-620. https://doi.org/10.1002/(SICI)1098-1063(1996)6:6<601::AID-HIPO5>3.0.CO;2-J

Rosburg, T., Trautner, P., Ludowig, E., Schaller, C., Kurthen, M., Elger, C.E., Boutros, N.N., 2007. Hippocampal event-related potentials to tone duration deviance in a passive oddball paradigm in humans. NeuroImage 37, 274-281. https://doi.org/10.1016/j.neuroimage.2007.05.002

Ross, R.T., Orr, W.B., Holland, P.C., Berger, T.W., 1984. Hippocampectomy Disrupts Acquisition and Retention of Learned Conditional Responding. Behavioral Neuroscience 98, 211-225.

Rothschild, G., Eban, E., Frank, L.M., 2017. A cortical-hippocampal-cortical loop of information processing during memory consolidation. Nature Neuroscience 20, 251-259. https://doi.org/10.1038/nn.4457

Rubin, R.D., Brown-Schmidt, S., Duff, M.C., Tranel, D., Cohen, N.J., 2011. How Do I Remember That I Know You Know That I Know? Psychological Science 22, 1574-1582. https://doi.org/10.1177/0956797611418245

Rudy, J.W., 2009. Context representations, context functions, and the parahippocampal-hippocampal system. Learning \& Memory 16, 573-585. https://doi.org/10.1101/lm.1494409

Rudy, J.W., O’Reilly, R.C., 2001. Conjunctive representations, the hippocampus, and contextual fear conditioning. Cognitive, Affective, \& Behavioral Neuroscience 1, 66-82. https://doi.org/10.3758/CABN.1.1.66

Rummell, B.P., Klee, J.L., Sigurdsson, T., 2016. Attenuation of Responses to Self-Generated Sounds in Auditory Cortical Neurons. The Journal of Neuroscience 36, 12010-12026. https://doi.org/10.1523/JNEUROSCI.1564-16.2016

Ruusuvirta, T., Korhonen, T., Arikoski, J., Kivirikko, K., 1996. ERPs to pitch changes: A result of reduced responses to standard tones in rabbits. NeuroReport 7, 413-416.

Ruusuvirta, T., Lipponen, A., Pellinen, E., Penttonen, M., Astikainen, P., 2013. Auditory Cortical and Hippocampal-System Mismatch Responses to Duration Deviants in Urethane-Anesthetized Rats. PLoS ONE 8, e54624. https://doi.org/10.1371/journal.pone.0054624

Sakurai, Y., 2002. Coding of auditory temporal and pitch information by hippocampal individual cells and cell assemblies in the rat. Neuroscience 115, 1153-1163. https://doi.org/10.1016/S0306-4522(02)00509-2

Salimpoor, V.N., van den Bosch, I., Kovacevic, N., McIntosh, A.R., Dagher, A., Zatorre, R.J., 2013. Interactions Between the Nucleus Accumbens and Auditory Cortices Predict Music Reward Value. Science 340, 216-219. https://doi.org/10.1126/science.1231059

Säljö, A., Bao, F., Haglid, K.G., Hansson, H.-A., 2000. Blast Exposure Causes Redistribution of Phosphorylated Neurofilament Subunits in Neurons of the Adult Rat Brain. Journal of Neurotrauma 17, 719-726. https://doi.org/10.1089/089771500415454

Säljö, A., Bao, F., Jingshan, S., Hamberger, A., Hansson, H.-A., Haglid, K.G., 2002. Exposure to Short-Lasting Impulse Noise Causes Neuronal c-Jun Expression and Induction of Apoptosis in the Adult Rat Brain. Journal of Neurotrauma 19, 985-991. https://doi.org/10.1089/089771502320317131

Samson, S., Peretz, I., 2005. Effects of Prior Exposure on Music Liking and Recognition in Patients with Temporal Lobe Lesions. Annals of the New York Academy of Sciences 1060, 419-428. https://doi.org/10.1196/annals.1360.035

Samson, S., Zatorre, R.J., 1994. Contribution of the right temporal lobe to musical timbre discrimination. Neuropsychologia 32, 231-240. https://doi.org/10.1016/0028-3932(94)90008-6

Samson, S., Zatorre, R.J., 1992. Learning and retention of melodic and verbal information after unilateral temporal lobectomy. Neuropsychologia 30, 815-826. https://doi.org/10.1016/0028-3932(92)90085-Z

Samson, S., Zatorre, R.J., 1991. Recognition Memory for Text and Melody of Songs After Unilateral Temporal Lobe Lesion Evidence for Dual Encoding. Journal of Experimental Psychology: Learning, Memory, and Cognition 17, $793-804$.

Sander, D., Grandjean, D., Pourtois, G., Schwartz, S., Seghier, M.L., Scherer, K.R., Vuilleumier, P., 2005. Emotion and attention interactions in social cognition: Brain regions involved in processing anger prosody. NeuroImage 28, 848-858. https://doi.org/10.1016/j.neuroimage.2005.06.023 
Sanyal, T., Kumar, V., Nag, T.C., Jain, S., Sreenivas, V., Wadhwa, S., 2013a. Prenatal Loud Music and Noise: Differential Impact on Physiological Arousal, Hippocampal Synaptogenesis and Spatial Behavior in One Day-Old Chicks. PLoS ONE 8, e67347. https://doi.org/10.1371/journal.pone.0067347

Sanyal, T., Palanisamy, P., Nag, T.C., Roy, T.S., Wadhwa, S., 2013b. Effect of prenatal loud music and noise on total number of neurons and glia, neuronal nuclear area and volume of chick brainstem auditory nuclei, field $\mathrm{L}$ and hippocampus: A stereological investigation. International Journal of Developmental Neuroscience 31, 234-244. https://doi.org/10.1016/j.ijdevneu.2013.02.004

Savelli, F., Knierim, J.J., 2019. Origin and role of path integration in the cognitive representations of the hippocampus: computational insights into open questions. Journal of Experimental Biology 222, jeb188912. https://doi.org/10.1242/jeb.188912

Schaette, R., Kempter, R., 2006. Development of tinnitus-related neuronal hyperactivity through homeostatic plasticity after hearing loss: a computational model. European Journal of Neuroscience 23, 3124-3138. https://doi.org/10.1111/j.1460-9568.2006.04774.x

Schapiro, A.C., Gregory, E., Landau, B., McCloskey, M., Turk-Browne, N.B., 2014. The Necessity of the Medial Temporal Lobe for Statistical Learning. Journal of Cognitive Neuroscience 26, 1736-1747. https://doi.org/10.1162/jocn_a_00578

Schapiro, A.C., Kustner, L.V., Turk-Browne, N.B., 2012. Shaping of Object Representations in the Human Medial Temporal Lobe Based on Temporal Regularities. Current Biology 22, 1622-1627. https://doi.org/10.1016/j.cub.2012.06.056

Schapiro, A.C., Turk-Browne, N.B., Botvinick, M.M., Norman, K.A., 2016. Complementary learning systems within the hippocampus: A neural network modeling approach to reconciling episodic memory with statistical learning. bioRxiv. https://doi.org/10.1101/051870

Schecklmann, M., Landgrebe, M., Poeppl, T.B., Kreuzer, P., Männer, P., Marienhagen, J., Wack, D.S., Kleinjung, T., Hajak, G., Langguth, B., 2013. Neural correlates of tinnitus duration and Distress: A positron emission tomography study. Human Brain Mapping 34, 233-240. https://doi.org/10.1002/hbm.21426

Schmaltz, L.W., Theios, J., 1972. Acquisition and extinction of a classically conditioned response in hippocampectomized rabbits (Oryctolaous Cuniculus). Journal of Comparative and Physiological Psychology 79, 328-333.

Schmithorst, V.J., 2005. Separate cortical networks involved in music perception: preliminary functional MRI evidence for modularity of music processing. NeuroImage 25, 444-451. https://doi.org/10.1016/j.neuroimage.2004.12.006

Schmithorst, V.J., Holland, S.K., Plante, E., 2006. Cognitive modules utilized for narrative comprehension in children: a functional magnetic resonance imaging study. NeuroImage 29, 254-266. https://doi.org/10.1016/j.neuroimage.2005.07.020

Schnupp, J.W.H., Honey, C., Willmore, B.D.B., 2013. Neural Correlates of Auditory Object Perception, in: Cohen, Y.E., Popper, A.N., Fay, R.R. (Eds.), Neural Correlates of Auditory Cognition, Springer Handbook of Auditory Research. Springer New York, New York, NY, pp. 115-149. https://doi.org/10.1007/978-1-4614-2350-8_5

Schwartzbaum, J.S., Thompson, J.B., Kellicutt, M.H., 1964. Auditory frequency discrimination and generalization following lesions of the amygdaloid area in rats. Journal of Comparative and Physiological Psychology 57, 257-266. https://doi.org/10.1037/h0039921

Schwenzer, M., Mathiak, K., 2011. Numeric aspects in pitch identification: an fMRI study. BMC Neuroscience 12. https://doi.org/10.1186/1471-2202-12-26

Scoville, W.B., Milner, B., 1957. Loss of recent memory after bilateral hippocampal lesions. Journal of Neurology, Neurosurgery \& Psychiatry 20, 11-21.

Sedley, W., Friston, K.J., Gander, P.E., Kumar, S., Griffiths, T.D., 2016. An Integrative Tinnitus Model Based on Sensory Precision. Trends in Neurosciences 39, 799-812. https://doi.org/10.1016/j.tins.2016.10.004

Sedley, W., Gander, P.E., Kumar, S., Oya, H., Kovach, C.K., Nourski, K.V., Kawasaki, H., Howard, M.A., Griffiths, T.D., 2015. Intracranial Mapping of a Cortical Tinnitus System using Residual Inhibition. Current Biology 25, 1208-1214. https://doi.org/10.1016/j.cub.2015.02.075

Sekeres, M.J., Winocur, G., Moscovitch, M., Anderson, J.A.E., Pishdadian, S., Martin Wojtowicz, J., St-Laurent, M., McAndrews, M.P., Grady, C.L., 2018. Changes in patterns of neural activity underlie a time-dependent transformation of memory in rats and humans. Hippocampus 28, 745-764. https://doi.org/10.1002/hipo.23009

Shan, K.Q., Lubenov, E.V., Papadopoulou, M., Siapas, A.G., 2016. Spatial tuning and brain state account for dorsal hippocampal CA1 activity in a non-spatial learning task. eLife 5, e14321. https://doi.org/10.7554/eLife.14321

Shen, Y., Hu, H., Fan, C., Wang, Q., Zou, T., Ye, B., Xiang, M., 2021. Sensorineural hearing loss may lead to dementia-related pathological changes in hippocampal neurons. Neurobiology of Disease 105408. https://doi.org/10.1016/j.nbd.2021.105408

Shergill, S.S., 2001. Modality specific neural correlates of auditory and somatic hallucinations. Journal of Neurology, Neurosurgery \& Psychiatry 71, 688-690. https://doi.org/10.1136/jnnp.71.5.688

Shergill, S.S., Brammer, M.J., Williams, S.C.R., Murray, R.M., McGuire, P.K., 2000. Mapping Auditory Hallucinations in Schizophrenia Using Functional Magnetic Resonance Imaging. Archives of General Psychiatry 57, 1033. https://doi.org/10.1001/archpsyc.57.11.1033

Shigeto, H., 2021. Hippocampal modulation of auditory processing in epilepsy. Neurol. Clin. Neurosci. 9, 17-23. https://doi.org/10.1111/ncn3.12470

Shimbo, A., Izawa, E.-I., Fujisawa, S., 2021. Scalable representation of time in the hippocampus. Sci. Adv. 7, eabd7013. https://doi.org/10.1126/sciadv.abd7013

Shin, J., 2011. The interrelationship between movement and cognition: Theta rhythm and the P300 event-related potential. Hippocampus 21, 744-752. https://doi.org/10.1002/hipo.20792

Shinba, T., 1999. Neuronal firing activity in the dorsal hippocampus during the auditory discrimination oddball task in awake rats: relation to event-related potential generation. Cognitive Brain Research 8, 241-250. https://doi.org/10.1016/S09266410(99)00026-9

Shinba, T., Andow, Y., Shinozaki, T., Ozawa, N., Yamamoto, K., 1996. Event-related potentials in the dorsal hippocampus of 
rats during an auditory discrimination paradigm. Electroencephalography and clinical Neurophysiology 6.

Shulman, A., 1995. A Final Common Pathway for Tinnitus The Medial Temporal Lobe System 1, 12.

Shulman, A., Strashun, A.M., Afriyie, M., Aronson, F., Abel, W., Goldstein, B., 1995. SPECT imaging of brain and tinnitus neurotologic/neurologic implications. International Tinnitus Journal 1, 13-29.

Silbersweig, D.A., Stern, E., Frith, C., Cahill, C., Holmes, A., Grootoonk, S., Seaward, J., McKenna, P., Chua, S.E., Schnorr, L., Jones, T., Frackowiak, R.S.J., 1995. A functional neuroanatomy of hallucinations in schizophrenia. Nature 378.

Simon, J.Z., 2015. The encoding of auditory objects in auditory cortex: Insights from magnetoencephalography. International Journal of Psychophysiology 95, 184-190. https://doi.org/10.1016/j.ijpsycho.2014.05.005

Singer, W., Zuccotti, A., Jaumann, M., Lee, S.C., Panford-Walsh, R., Xiong, H., Zimmermann, U., Franz, C., Geisler, H.-S., Köpschall, I., Rohbock, K., Varakina, K., Verpoorten, S., Reinbothe, T., Schimmang, T., Rüttiger, L., Knipper, M., 2013. Noise-Induced Inner Hair Cell Ribbon Loss Disturbs Central Arc Mobilization: A Novel Molecular Paradigm for Understanding Tinnitus. Molecular Neurobiology 47, 261-279. https://doi.org/10.1007/s12035-012-8372-8

Sliwa, J., Planté, A., Duhamel, J.-R., Wirth, S., 2014. Independent Neuronal Representation of Facial and Vocal Identity in the Monkey Hippocampus and Inferotemporal Cortex. Cerebral Cortex 26, 950-966. https://doi.org/10.1093/cercor/bhu257

Smith, M.E., Halgren, E., Sokolik, M., Baudena, P., Musolino, A., Liegeois-Chauvel, C., Chauvel, P., 1990. The intracranial topography of the P3 event-related potential elicited during auditory oddball. Electroencephalography and Clinical Neurophysiology 76, 235-248. https://doi.org/10.1016/0013-4694(90)90018-F

Smith, M.E., Stapleton, J.M., Halgren, E., 1986. Human medial temporal lobe potentials evoked in memory and language tasks. Electroencephalography and Clinical Neurophysiology 63, 145-159.

Snyder, J.S., Elhilali, M., 2017. Recent advances in exploring the neural underpinnings of auditory scene perception. Ann. N.Y. Acad. Sci. 1396, 39-55. https://doi.org/10.1111/nyas.13317

Solomon, E.A., Lega, B.C., Sperling, M.R., Kahana, M.J., 2019. Hippocampal theta codes for distances in semantic and temporal spaces. Proc Natl Acad Sci USA 116, 24343-24352. https://doi.org/10.1073/pnas.1906729116

Solomon, P.R., 1977. Role of the hippocampus in blocking and conditioned inhibition of the rabbit's nictitating membrane response. Journal of Comparative and Physiological Psychology 91, 407-417. https://doi.org/10.1037/h0077330

Solomon, P.R., Moore, J.W., 1975. Latent Inhibition and Stimulus Generalization of the Classically Conditioned Nictitating Membrane Response in Rabbits (Oryctolagus cuniculus) Following Dorsal Hippocampal Ablation. Journal of Comparative and Physiological Psychology 89, 1192-1203.

Solomon, P.R., Schaaf, E.R.V., College, W., Thompson, R.F., Weisz, D.J., 1986. Hippocampus and Trace Conditioning of the Rabbit's Classically Conditioned Nictitating Membrane Response. Behavioral Neuroscience 100, 729-744.

Sommer, I.E.C., Diederen, K.M.J., Blom, J.-D., Willems, A., Kushan, L., Slotema, K., Boks, M.P.M., Daalman, K., Hoek, H.W., Neggers, S.F.W., Kahn, R.S., 2008. Auditory verbal hallucinations predominantly activate the right inferior frontal area. Brain 131, 3169-3177. https://doi.org/10.1093/brain/awn251

Song, J.-J., De Ridder, D., Van de Heyning, P., Vanneste, S., 2012. Mapping Tinnitus-Related Brain Activation: An ActivationLikelihood Estimation Metaanalysis of PET Studies. Journal of Nuclear Medicine 53, 1550-1557. https://doi.org/10.2967/jnumed.112.102939

Squire, L., Zola-Morgan, S., 1991. The medial temporal lobe memory system. Science 253, 1380-1386. https://doi.org/10.1126/science.1896849

Squire, L.R., Alvarez, P., 1995. Retrograde amnesia and memory consolidation: a neurobiological perspective. Current Opinion in Neurobiology 5, 169-177. https://doi.org/10.1016/0959-4388(95)80023-9

Squire, L.R., Schmolck, H., Stark, S.M., 2001. Impaired Auditory Recognition Memory in Amnesic Patients with Medial Temporal Lobe Lesions. Learning \& Memory 8, 252-256. https://doi.org/10.1101/lm.42001

Sreenivasan, K.K., D’Esposito, M., 2019. The what, where and how of delay activity. Nature Reviews Neuroscience 20, $466-481$. https://doi.org/10.1038/s41583-019-0176-7

Stachenfeld, K.L., Botvinick, M.M., Gershman, S.J., 2017. The hippocampus as a predictive map. Nature Neuroscience 20, 16431653. https://doi.org/10.1038/nn.4650

Stapleton, J.M., Halgren, E., Moreno, K.A., 1987. Endogenous potentials after anterior temporal lobectomy. Neuropsychologia 25, 549-557. https://doi.org/10.1016/0028-3932(87)90079-0

Stefanacci, L., Buffalo, E.A., Schmolck, H., Squire, L.R., 2000. Profound Amnesia After Damage to the Medial Temporal Lobe: A Neuroanatomical and Neuropsychological Profile of Patient E. P. The Journal of Neuroscience 20, 7024-7036. https://doi.org/10.1523/JNEUROSCI.20-18-07024.2000

St-Laurent, M., Moscovitch, M., Jadd, R., McAndrews, M.P., 2014. The perceptual richness of complex memory episodes is compromised by medial temporal lobe damage. Hippocampus 24, 560-576. https://doi.org/10.1002/hipo.22249

Sundaramahalingam, M., Ramasundaram, S., Rathinasamy, S.D., Natarajan, R.P., Somasundaram, T., 2013. Role of Acorus calamus and $\alpha$-asarone on Hippocampal Dependent Memory in Noise Stress Exposed Rats. Pakistan Journal of Biological Sciences 16, 770-778. https://doi.org/10.3923/pjbs.2013.770.778

Sutton, S., Braren, M., Zubin, J., John, E.R., 1965. Evoked-potential correlates of stimulus uncertainty. Science 150, 1187-1188.

Suzuki, K., Takei, N., Toyoda, T., Iwata, Y., Hoshino, R., Minabe, Y., Mori, N., 2003. Auditory hallucinations and cognitive impairment in a patient with a lesion restricted to the hippocampus. Schizophrenia Research 64, 87-89. https://doi.org/10.1016/S0920-9964(02)00386-9

Suzuki, W.L., Amaral, D.G., 1994. Perirhinal and parahippocampal cortices of the macaque monkey: Cortical afferents. The Journal of Comparative Neurology 350, 497-533. https://doi.org/10.1002/cne.903500402

Svoboda, E., Levine, B., 2009. The Effects of Rehearsal on the Functional Neuroanatomy of Episodic Autobiographical and Semantic Remembering: A Functional Magnetic Resonance Imaging Study. Journal of Neuroscience 29, 3073-3082. https://doi.org/10.1523/JNEUROSCI.3452-08.2009

Swanson, A.M., Isaacson, R.L., 1967. Hippocampal ablation and performance during withdrawal of reinforcement. Journal of Comparative and Physiological Psychology 64, 30-35. https://doi.org/10.1037/h0024797 
Szameitat, D.P., Kreifelts, B., Alter, K., Szameitat, A.J., Sterr, A., Grodd, W., Wildgruber, D., 2010. It is not always tickling: Distinct cerebral responses during perception of different laughter types. NeuroImage 53, 1264-1271. https://doi.org/10.1016/j.neuroimage.2010.06.028

Tachibana, H., Miyata, Y., Takeda, M., Minamoto, H., Sugita, M., Okita, T., 1999. Auditory event-related potentials in an amnesic patient with a left temporal lobe lesion. Journal of the Neurological Sciences 168, 52-56. https://doi.org/10.1016/S0022-510X(99)00182-3

Tae, W.-S., Yakunina, N., Lee, W.H., Ryu, Y.-J., Ham, H., Pyun, S.-B., Nam, E.-C., 2018. Changes in the regional shape and volume of subcortical nuclei in patients with tinnitus comorbid with mild hearing loss. Neuroradiology 60, 1203-1211. https://doi.org/10.1007/s00234-018-2093-2

Takahashi, S., Sakurai, Y., 2009. Sub-millisecond firing synchrony of closely neighboring pyramidal neurons in hippocampal CA1 of rats during delayed non-matching to sample task. Frontiers in Neural Circuits 3. https://doi.org/10.3389/neuro.04.009.2009

Takakura, H., Umeno, K., Tabuchi, E., Hori, E., Miyamoto, K., Aso, S., Watanabe, Y., Ono, T., Nishijo, H., 2003. Differential activation in the medial temporal lobe during a sound-sequence discrimination task across age in human subjects. Neuroscience 119, 517-532. https://doi.org/10.1016/S0306-4522(03)00193-3

Takebayashi, H., Takei, N., Suzuki, S., Mori, N., 2002. Unilateral auditory hallucinations in schizophrenia after damage to the right hippocampus. Schizophrenia Research 58, 329-331. https://doi.org/10.1016/S0920-9964(01)00399-1

Talk, A., Grasby, K., Rawson, T., Ebejer, J., 2016. Preconditioning of Spatial and Auditory Cues: Roles of the Hippocampus, Frontal Cortex, and Cue-Directed Attention. Brain Sciences 6, 63. https://doi.org/10.3390/brainsci6040063

Talk, A.C., Gandhi, C.C., Matzel, L.D., 2002. Hippocampal function during behaviorally silent associative learning: Dissociation of memory storage and expression. Hippocampus 12, 648-656. https://doi.org/10.1002/hipo.10098

Tamura, R., Ono, T., Fukuda, M., Nakamura, K., 1992. Spatial responsiveness of monkey hippocampal neurons to various visual and auditory stimuli. Hippocampus 2, 307-322. https://doi.org/10.1002/hipo.450020309

Tamura, R., Ono, T., Fukuda, M., Nakamura, K., 1990. Recognition of egocentric and allocentric visual and auditory space by neurons in the hippocampus of monkeys. Neuroscience Letters 109, 293-298. https://doi.org/10.1016/03043940(90)90010-7

Tang, W., Shin, J.D., Jadhav, S.P., 2021. Multiple time-scales of decision-making in the hippocampus and prefrontal cortex. eLife 10, e66227. https://doi.org/10.7554/eLife.66227

Taube, J., Muller, R., Ranck, J., 1990. Head-direction cells recorded from the postsubiculum in freely moving rats. I. Description and quantitative analysis. The Journal of Neuroscience 10, 420-435. https://doi.org/10.1523/JNEUROSCI.10-0200420.1990

Tavares, R.M., Mendelsohn, A., Grossman, Y., Williams, C.H., Shapiro, M., Trope, Y., Schiller, D., 2015. A Map for Social Navigation in the Human Brain. Neuron 87, 231-243. https://doi.org/10.1016/j.neuron.2015.06.011

Teki, S., Griffiths, T.D., 2016. Brain Bases of Working Memory for Time Intervals in Rhythmic Sequences. Frontiers in Neuroscience 10. https://doi.org/10.3389/fnins.2016.00239

Teki, S., Kumar, S., von Kriegstein, K., Stewart, L., Lyness, C.R., Moore, B.C.J., Capleton, B., Griffiths, T.D., 2012. Navigating the Auditory Scene: An Expert Role for the Hippocampus. Journal of Neuroscience 32, 12251-12257. https://doi.org/10.1523/JNEUROSCI.0082-12.2012

Terada, S., Sakurai, Y., Nakahara, H., Fujisawa, S., 2017. Temporal and Rate Coding for Discrete Event Sequences in the Hippocampus. Neuron 94, 1248-1262.e4. https://doi.org/10.1016/j.neuron.2017.05.024

Teyler, T.J., DiScenna, P., 1986. The Hippocampal Memory Indexing Theory. Behavioral Neuroscience 100, 147-154.

Thompson, R.F., 2005. In Search of Memory Traces. Annual Review of Psychology 56, 1-23. https://doi.org/10.1146/annurev.psych.56.091103.070239

Thompson, W.F., Schellenberg, E.G., Husain, G., 2001. Arousal, Mood, and The Mozart Effect. Psychol Sci 12, $248-251$. https://doi.org/10.1111/1467-9280.00345

Tierney, T.M., Levy, A., Barry, D.N., Meyer, S.S., Shigihara, Y., Everatt, M., Mellor, S., Lopez, J.D., Bestmann, S., Holmes, N., Roberts, G., Hill, R.M., Boto, E., Leggett, J., Shah, V., Brookes, M.J., Bowtell, R., Maguire, E.A., Barnes, G.R., 2021. Mouth magnetoencephalography: A unique perspective on the human hippocampus. NeuroImage 225, 117443. https://doi.org/10.1016/j.neuroimage.2020.117443

Tobia, M.J., Iacovella, V., Hasson, U., 2012. Multiple sensitivity profiles to diversity and transition structure in non-stationary input. NeuroImage 60, 991-1005. https://doi.org/10.1016/j.neuroimage.2012.01.041

Tolman, E.C., 1948. Cognitive maps in rats and men. Psychological Review 55, 189-208. https://doi.org/10.1037/h0061626

Tranel, D., Brady, D.R., Van Hoesen, G.W., Damasio, A.R., 1988. Parahippocampal projections to posterior auditory association cortex (area Tpt) in Old-World monkeys. Experimental Brain Research 70, 406-416. https://doi.org/10.1007/BF00248365

Trost, W., Ethofer, T., Zentner, M., Vuilleumier, P., 2012. Mapping Aesthetic Musical Emotions in the Brain. Cerebral Cortex 22, 2769-2783. https://doi.org/10.1093/cercor/bhr353

Tsao, A., Sugar, J., Lu, L., Wang, C., Knierim, J.J., Moser, M.-B., Moser, E.I., 2018. Integrating time from experience in the lateral entorhinal cortex. Nature 561, 57-62. https://doi.org/10.1038/s41586-018-0459-6

Tsivilis, D., Vann, S.D., Denby, C., Roberts, N., Mayes, A.R., Montaldi, D., Aggleton, J.P., 2008. A disproportionate role for the fornix and mammillary bodies in recall versus recognition memory. Nat Neurosci 11, 834-842. https://doi.org/10.1038/nn.2149

Turk-Browne, N.B., 2019. The hippocampus as a visual area organized by space and time: A spatiotemporal similarity hypothesis. Vision Research 165, 123-130. https://doi.org/10.1016/j.visres.2019.10.007

Tuwaig, M., Savard, M., Jutras, B., Poirier, J., Collins, D.L., Rosa-Neto, P., Fontaine, D., Breitner, J.C.S., 2017. Deficit in Central Auditory Processing as a Biomarker of Pre-Clinical Alzheimer's Disease. Journal of Alzheimer's Disease 60, 1589_ 1600.

Ueyama, T., Donishi, T., Ukai, S., Ikeda, Y., Hotomi, M., Yamanaka, N., Shinosaki, K., Terada, M., Kaneoke, Y., 2013. Brain 
Regions Responsible for Tinnitus Distress and Loudness: A Resting-State fMRI Study. PLoS ONE 8, e67778. https://doi.org/10.1371/journal.pone.0067778

Ulanovsky, N., Moss, C.F., 2011. Dynamics of hippocampal spatial representation in echolocating bats. Hippocampus 21, 150161. https://doi.org/10.1002/hipo.20731

Ulanovsky, N., Moss, C.F., 2008. What the bat's voice tells the bat's brain. Proceedings of the National Academy of Sciences 105, 8491-8498. https://doi.org/10.1073/pnas.0703550105

Ulanovsky, N., Moss, C.F., 2007. Hippocampal cellular and network activity in freely moving echolocating bats. Nature Neuroscience 10, 224-233. https://doi.org/10.1038/nn1829

Umbach, G., Kantak, P., Jacobs, J., Kahana, M., Pfeiffer, B.E., Sperling, M., Lega, B., 2020. Time cells in the human hippocampus and entorhinal cortex support episodic memory. Proc Natl Acad Sci USA 117, 28463-28474. https://doi.org/10.1073/pnas.2013250117

Ungiadze, A.A., 1967. Electrical activity of the hippocampus during peripheral stimulation. Neuroscience Translations 1, 136142. https://doi.org/10.1007/BF01124392

Uran, S.L., Aon-Bertolino, M.L., Caceres, L.G., Capani, F., Guelman, L.R., 2012. Rat hippocampal alterations could underlie behavioral abnormalities induced by exposure to moderate noise levels. Brain Research 1471, 1-12. https://doi.org/10.1016/j.brainres.2012.06.022

Uran, S.L., Caceres, L.G., Guelman, L.R., 2010. Effects of loud noise on hippocampal and cerebellar-related behaviors. Brain Research 1361, 102-114. https://doi.org/10.1016/j.brainres.2010.09.022

Uran, S.L., Gómez-Casati, M.E., Guelman, L.R., 2014. Long-term recovery from hippocampal-related behavioral and biochemical abnormalities induced by noise exposure during brain development. Evaluation of auditory pathway integrity. International Journal of Developmental Neuroscience 37, 41-51. https://doi.org/10.1016/j.ijdevneu.2014.06.002

Urgolites, Z.J., Wixted, J.T., Goldinger, S.D., Papesh, M.H., Treiman, D.M., Squire, L.R., Steinmetz, P.N., 2020. Spiking activity in the human hippocampus prior to encoding predicts subsequent memory. Proc Natl Acad Sci USA 117, 1376713770. https://doi.org/10.1073/pnas.2001338117

Valtonen, J., Gregory, E., Landau, B., McCloskey, M., 2014. New Learning of Music after Bilateral Medial Temporal Lobe Damage: Evidence from an Amnesic Patient. Frontiers in Human Neuroscience 8. https://doi.org/10.3389/fnhum.2014.00694

van de Ven, V., Waldorp, L., Christoffels, I., 2020. Hippocampus plays a role in speech feedback processing. NeuroImage 223, 117319. https://doi.org/10.1016/i.neuroimage.2020.117319

van Dongen, E.V., Takashima, A., Barth, M., Zapp, J., Schad, L.R., Paller, K.A., Fernandez, G., 2012. Memory stabilization with targeted reactivation during human slow-wave sleep. Proceedings of the National Academy of Sciences 109, 1057510580. https://doi.org/10.1073/pnas.1201072109

van Strien, N.M., Cappaert, N.L.M., Witter, M.P., 2009. The anatomy of memory: an interactive overview of the parahippocampal-hippocampal network. Nat Rev Neurosci 10, 272-282. https://doi.org/10.1038/nrn2614

Vargha-Khadem, F., Gadian, D.G., Watkins, K.E., Connelly, A., Van Paesschen, W., Mishkin, M., 1997. Differential Effects of Early Hippocampal Pathology on Episodic and Semantic Memory. Science 277, 376-380. https://doi.org/10.1126/science.277.5324.376

Vaudano, E., Legg, C.R., Glickstein, M., 1991. Afferent and Efferent Connections of Temporal Association Cortex in the Rat: A Horseradish Peroxidase Study. European Journal of Neuroscience 3, 317-330. https://doi.org/10.1111/j.14609568.1991.tb00818.x

Velasco, M., Velasco, F., Velasco, A.L., Almanza, X., Olvera, A., 1986. Subcortical correlates of the P300 potential complex in man to auditory stimuli. Electroencephalography and Clinical Neurophysiology 64, 199-210. https://doi.org/10.1016/0013-4694(86)90166-5

Viganò, S., Piazza, M., 2020. Distance and Direction Codes Underlie Navigation of a Novel Semantic Space in the Human Brain. The Journal of Neuroscience 40, 2727-2736. https://doi.org/10.1523/JNEUROSCI.1849-19.2020

Vinnik, E., Antopolskiy, S., Itskov, P.M., Diamond, M.E., 2012. Auditory stimuli elicit hippocampal neuronal responses during sleep. Frontiers in Systems Neuroscience 6. https://doi.org/10.3389/fnsys.2012.00049

Vinogradova, O.S., 2001. Hippocampus as comparator: Role of the two input and two output systems of the hippocampus in selection and registration of information. Hippocampus 11, 578-598. https://doi.org/10.1002/hipo.1073

Vinogradova, O.S., 1975a. Functional Organization of the Limbic System in the Process of Registration of Information: Facts and Hypotheses, in: Isaacson, R.L., Pribram, K.H. (Eds.), The Hippocampus. Springer US, Boston, MA, pp. 3-69. https://doi.org/10.1007/978-1-4684-2979-4_1

Vinogradova, O.S., 1975b. Functional organization of the limbic system in the process of registration of information: Facts and hypotheses, in: The Hippocampus. Volume 2: Neurophysiology and Behavior. Plenum Press, New York, NY, pp. 3 70.

Vinogradova, O.S., Semyonova, T.P., Konovalov, V.Ph., 1970. Trace phenomena in single neurons of hippocampus and mammiliary bodies, in: Biology of Memory. Elsevier, pp. 191-221. https://doi.org/10.1016/B978-0-12-5643504.50021-X

Vohs, J.L., Andrew Chambers, R., Krishnan, G.P., O’Donnell, B.F., Berg, S., Morzorati, S.L., 2010. GABAergic modulation of the $40 \mathrm{~Hz}$ auditory steady-state response in a rat model of schizophrenia. The International Journal of Neuropsychopharmacology 13, 487. https://doi.org/10.1017/S1461145709990307

Vohs, J.L., Chambers, R.A., Krishnan, G.P., O\&rsquo;Donnell, B.F., Hetrick, W.P., Kaiser, S.T., Berg, S., Morzorati, S.L., 2009. Auditory Sensory Gating in the Neonatal Ventral Hippocampal Lesion Model of Schizophrenia. Neuropsychobiology 60, 12-22. https://doi.org/10.1159/000234813

Vohs, J.L., Chambers, R.A., O’Donnell, B.F., Krishnan, G.P., Morzorati, S.L., 2012. Auditory steady state responses in a schizophrenia rat model probed by excitatory/inhibitory receptor manipulation. International Journal of Psychophysiology 86, 136-142. https://doi.org/10.1016/j.ijpsycho.2012.04.002 
Volkow, N.D., Wolf, A.P., van Gelder, P., Brodie, J.D., Overall, J.E., Cancro, R., Gomez-Mont, F., 1987. Phenomological correlates of metabolic activity in 18 patients with chronic schizophrenia. American Journal of Psychiatry 144, 151158.

Wahlstrom, K.L., Huff, M.L., Emmons, E.B., Freeman, J.H., Narayanan, N.S., McIntyre, C.K., LaLumiere, R.T., 2018. Basolateral Amygdala Inputs to the Medial Entorhinal Cortex Selectively Modulate the Consolidation of Spatial and Contextual Learning. J. Neurosci. 38, 2698-2712. https://doi.org/10.1523/JNEUROSCI.2848-17.2018

Wang, J.X., Rogers, L.M., Gross, E.Z., Ryals, A.J., Dokucu, M.E., Brandstatt, K.L., Hermiller, M.S., Voss, J.L., 2014. Targeted enhancement of cortical-hippocampal brain networks and associative memory. Science 345, 1054-1057. https://doi.org/10.1126/science. 1252900

Wang, S., Yu, Y., Feng, Y., Zou, F., Zhang, X., Huang, J., Zhang, Y., Zheng, X., Huang, X.-F., Zhu, Y., Liu, Y., 2016. Protective effect of the orientin on noise-induced cognitive impairments in mice. Behavioural Brain Research 296, 290-300. https://doi.org/10.1016/j.bbr.2015.09.024

Wang, S.-F., Ritchey, M., Libby, L.A., Ranganath, C., 2016. Functional connectivity based parcellation of the human medial temporal lobe. Neurobiology of Learning and Memory 12.

Warren, J.D., Jennings, A.R., Griffiths, T.D., 2005. Analysis of the spectral envelope of sounds by the human brain. NeuroImage 24, 1052-1057. https://doi.org/10.1016/j.neuroimage.2004.10.031

Watanabe, T., Yagishita, S., Kikyo, H., 2008. Memory of music: Roles of right hippocampus and left inferior frontal gyrus. NeuroImage 39, 483-491. https://doi.org/10.1016/j.neuroimage.2007.08.024

Weisberg, S.M., Ekstrom, A.D., 2021. Hippocampal volume and navigational ability: The map(ping) is not to scale. Neuroscience \& Biobehavioral Reviews 126, 102-112. https://doi.org/10.1016/j.neubiorev.2021.03.012

Weiss, C., Kronforst-Collins, M.A., Disterhoft, J.F., 1996. Activity of hippocampal pyramidal neurons during trace eyeblink conditioning. Hippocampus 6, 192-209. https://doi.org/10.1002/(SICI)1098-1063(1996)6:2<192::AIDHIPO9>3.0.CO;2-R

Weitz, A.J., Fang, Z., Lee, H.J., Fisher, R.S., Smith, W.C., Choy, M., Liu, J., Lin, P., Rosenberg, M., Lee, J.H., 2015. Optogenetic fMRI reveals distinct, frequency-dependent networks recruited by dorsal and intermediate hippocampus stimulations. NeuroImage 107, 229-241. https://doi.org/10.1016/j.neuroimage.2014.10.039

Weston, P.S.J., Nicholas, J.M., Henley, S.M.D., Liang, Y., Macpherson, K., Donnachie, E., Schott, J.M., Rossor, M.N., Crutch, S.J., Butler, C.R., Zeman, A.Z., Fox, N.C., 2018. Accelerated long-term forgetting in presymptomatic autosomal dominant Alzheimer's disease: a cross-sectional study. The Lancet Neurology 17, 123-132. https://doi.org/10.1016/S1474-4422(17)30434-9

Whittington, J.C.R., Muller, T.H., Mark, S., Chen, G., Barry, C., Burgess, N., Behrens, T.E.J., 2020. The Tolman-Eichenbaum Machine: Unifying Space and Relational Memory through Generalization in the Hippocampal Formation. Cell 183, 1249-1263.e23. https://doi.org/10.1016/j.cell.2020.10.024

Wickelgren, W.A., 1968. Sparing of short-term memory in an amnesic patient: Implications for strength theory of memory 10.

Wiethoff, S., Wildgruber, D., Kreifelts, B., Becker, H., Herbert, C., Grodd, W., Ethofer, T., 2008. Cerebral processing of emotional prosody - influence of acoustic parameters and arousal. NeuroImage 39, 885-893. https://doi.org/10.1016/j.neuroimage.2007.09.028

Wilkins, R.W., Hodges, D.A., Laurienti, P.J., Steen, M., Burdette, J.H., 2014. Network Science and the Effects of Music Preference on Functional Brain Connectivity: From Beethoven to Eminem. Scientific Reports 4. https://doi.org/10.1038/srep06130

Wills, T.J., Lever, C., Cacucci, F., Burgess, N., O'Keefe, J., 2005. Attractor dynamics in the hippocampal representation of the local environment. Science 308, 873-876. https://doi.org/10.1126/science.1108905

Winocur, G., Moscovitch, M., 2011. Memory Transformation and Systems Consolidation. Journal of the International Neuropsychological Society 17, 766-780. https://doi.org/10.1017/S1355617711000683

Witt, J.-A., Coras, R., Schramm, J., Becker, A.J., Elger, C.E., Blümcke, I., Helmstaedter, C., 2014. The overall pathological status of the left hippocampus determines preoperative verbal memory performance in left mesial temporal lobe epilepsy: Hippocampal Neuronal Cell Densities and Memory Functions. Hippocampus 24, 446-454. https://doi.org/10.1002/hipo.22238

Witter, M.P., Amaral, D.G., 2021. The entorhinal cortex of the monkey: VI. Organization of projections from the hippocampus, subiculum, presubiculum, and parasubiculum. J Comp Neurol 529, 828-852. https://doi.org/10.1002/cne.24983

Woods, N.I., Stefanini, F., Apodaca-Montano, D.L., Tan, I.M.C., Biane, J.S., Kheirbek, M.A., 2020. The Dentate Gyrus Classifies Cortical Representations of Learned Stimuli. Neuron 107, 173-184.e6. https://doi.org/10.1016/j.neuron.2020.04.002

Woollett, K., Maguire, E.A., 2011. Acquiring “the Knowledge” of London’s Layout Drives Structural Brain Changes. Current Biology 21, 2109-2114. https://doi.org/10.1016/j.cub.2011.11.018

Wu, G., Yao, J., Hu, B., Zhang, H., Li, Y., Li, X., Li, Q., Sui, J., 2013. Reevaluating the Role of the Hippocampus in Delay Eyeblink Conditioning. PLoS ONE 8, e71249. https://doi.org/10.1371/journal.pone.0071249

Xiao, C., Liu, Y., Xu, J., Gan, X., Xiao, Z., 2018. Septal and Hippocampal Neurons Contribute to Auditory Relay and Fear Conditioning. Frontiers in Cellular Neuroscience 12. https://doi.org/10.3389/fncel.2018.00102

Xing, Y., Chen, W., Wang, Y., Jing, W., Gao, S., Guo, D., Xia, Y., Yao, D., 2016a. Music exposure improves spatial cognition by enhancing the BDNF level of dorsal hippocampal subregions in the developing rats. Brain Research Bulletin 121, 131137. https://doi.org/10.1016/j.brainresbull.2016.01.009

Xing, Y., Xia, Y., Kendrick, K., Liu, X., Wang, M., Wu, D., Yang, H., Jing, W., Guo, D., Yao, D., 2016b. Mozart, Mozart Rhythm and Retrograde Mozart Effects: Evidences from Behaviours and Neurobiology Bases. Scientific Reports 6. https://doi.org/10.1038/srep18744

Yang, J., Hu, L., Song, T., Liu, Y., Wu, Q., Zhao, L., Liu, L., Zhao, X., Zhang, D., Huang, C., 2014a. Proteomic Changes in Female Rat Hippocampus Following Exposure to a Terrified Sound Stress. Journal of Molecular Neuroscience 53, 158-165. https://doi.org/10.1007/s12031-014-0242-6

Yang, J., Hu, L., Wu, Q., Liu, L., Zhao, L., Zhao, X., Song, T., Huang, C., 2014b. A terrified-sound stress induced proteomic 
changes in adult male rat hippocampus. Physiology \& Behavior 128, 32-38.

https://doi.org/10.1016/j.physbeh.2014.01.038

Yi, G.-L., Zhu, M.-Z., Cui, H.-C., Yuan, X.-R., Liu, P., Tang, J., Li, Y.-Q., Zhu, X.-H., 2022. A hippocampus dependent neural circuit loop underlying the generation of auditory mismatch negativity. Neuropharmacology 206, 108947. https://doi.org/10.1016/j.neuropharm.2022.108947

Yonelinas, A.P., 2013. The hippocampus supports high-resolution binding in the service of perception, working memory and long-term memory. Behavioural Brain Research 254, 34-44. https://doi.org/10.1016/j.bbr.2013.05.030

Yonelinas, A.P., Ranganath, C., Ekstrom, A.D., Wiltgen, B.J., 2019. A contextual binding theory of episodic memory: systems consolidation reconsidered. Nature Reviews Neuroscience. https://doi.org/10.1038/s41583-019-0150-4

Yoshiura, T., Zhong, J., Shibata, D.K., Kwok, W.E., Shrier, D.A., Numaguchi, Y., 1999. Functional MRI study of auditory and visual oddball tasks: NeuroReport 10,1683-1688. https://doi.org/10.1097/00001756-199906030-00011

Yu, C., Moss, C.F., 2022. Natural acoustic stimuli evoke selective responses in the hippocampus of passive listening bats. Hippocampus hipo.23407. https://doi.org/10.1002/hipo.23407

Yu, Y.-F., Zhai, F., Dai, C.-F., Hu, J.-J., 2011. The relationship between age-related hearing loss and synaptic changes in the hippocampus of C57BL/6J mice. Experimental Gerontology 46, 716-722. https://doi.org/10.1016/j.exger.2011.04.007

Zatorre, R.J., 1984. Musical Perception and Cerebral Function: A Critical Review. Music Perception 2, 196-221. https://doi.org/10.2307/40285291

Zeidman, P., Mullally, S.L., Maguire, E.A., 2015. Constructing, Perceiving, and Maintaining Scenes: Hippocampal Activity and Connectivity. Cerebral Cortex 25, 3836-3855. https://doi.org/10.1093/cercor/bhu266

Zevin, J.D., McCandliss, B.D., 2005. Dishabituation of the BOLD response to speech sounds. Behavioral and Brain Functions 12.

Zhang, G.-W., Sun, W.-J., Zingg, B., Shen, L., He, J., Xiong, Y., Tao, H.W., Zhang, L.I., 2018. A Non-canonical Reticular-Limbic Central Auditory Pathway via Medial Septum Contributes to Fear Conditioning. Neuron 97, 406-417.e4. https://doi.org/10.1016/j.neuron.2017.12.010

Zhang, H., Lin, S.-C., Nicolelis, M.A.L., 2011. A distinctive subpopulation of medial septal slow-firing neurons promote hippocampal activation and theta oscillations. Journal of Neurophysiology 106, 2749-2763. https://doi.org/10.1152/jn.00267.2011

Zhang, L., Wang, J., Sun, H., Feng, G., Gao, Z., 2022. Interactions between the hippocampus and the auditory pathway. Neurobiology of Learning and Memory 189, 107589. https://doi.org/10.1016/j.nlm.2022.107589

Zhang, L., Wu, C., Martel, D.T., West, M., Sutton, M.A., Shore, S.E., 2021. Noise Exposure Alters Glutamatergic and GABAergic Synaptic Connectivity in the Hippocampus and Its Relevance to Tinnitus. Neural Plasticity 2021, 1-16. https://doi.org/10.1155/2021/8833087

Zhang, L., Wu, C., Martel, D.T., West, M., Sutton, M.A., Shore, S.E., 2018. Remodeling of cholinergic input to the hippocampus after noise exposure and tinnitus induction in Guinea pigs. Hippocampus hipo.23058. https://doi.org/10.1002/hipo.23058

Zhao, H., Wang, L., Chen, Liang, Zhang, J., Sun, W., Salvi, R.J., Huang, Y.-N., Wang, M., Chen, Lin, 2018. Temporary conductive hearing loss in early life impairs spatial memory of rats in adulthood. Brain and Behavior 8, e01004. https://doi.org/10.1002/brb3.1004

Zhou, G., Olofsson, J.K., Koubeissi, M.Z., Menelaou, G., Rosenow, J., Schuele, S.U., Xu, P., Voss, J.L., Lane, G., Zelano, C., 2021. Human hippocampal connectivity is stronger in olfaction than other sensory systems. Progress in Neurobiology 201, 102027. https://doi.org/10.1016/j.pneurobio.2021.102027 